\title{
Vanishing cycles for formal schemes
}

\author{
Vladimir G. Berkovich * \\ Department of Theoretical Mathematics, The Weizmann Institute of Science, P.O.B. 26, 76100 Rehovot, \\ Israel
}

Oblatum 4-VI-1993 \& 6-VIII-1993

\section{Introduction}

Let $k$ be a non-Archimedean field, and let $\mathfrak{X}$ be a formal scheme locally finitely presented over the ring of integers $k^{\circ}$ (see $\S 1$ ). In this work we construct and study the vanishing cycles functor from the category of étale sheaves on the generic fibre $\mathfrak{X}_{\eta}$ of $\mathfrak{X}$ (which is a $k$-analytic space) to the category of étale sheaves on the closed fibre $\mathfrak{X}_{s}$ of $\mathfrak{X}$ (which is a scheme over the residue field of $k$ ). We prove that if $\mathfrak{X}$ is the formal completion $\widehat{\mathcal{X}}$ of a scheme $\mathcal{X}$ finitely presented over $k^{\circ}$ along the closed fibre, then the vanishing cycles sheaves of $\widehat{\mathcal{X}}$ are canonically isomorphic to those of $\mathcal{X}$ (as defined in [SGA7], Exp. XIII). In particular, the vanishing cycles sheaves of $\mathcal{X}$ depend only on $\hat{\mathcal{X}}$, and any morphism $\varphi: \hat{\mathcal{Y}} \rightarrow \widehat{\mathcal{X}}$ induces a homomorphism from the pullback of the vanishing cycles sheaves of $\mathcal{X}$ under $\varphi_{s}: \mathcal{Y}_{s} \rightarrow \mathcal{X}_{s}$ to those of $\mathcal{Y}$. Furthermore, we prove that, for each $\widehat{\mathcal{X}}$, one can find a nontrivial ideal of $k^{\circ}$ such that if two morphisms $\varphi, \psi: \hat{\mathcal{Y}} \rightarrow \widehat{\mathcal{X}}$ coincide modulo this ideal, then the homomorphisms between the vanishing cycles sheaves induced by $\varphi$ and $\psi$ coincide. These facts were conjectured by P. Deligne.

In $\S 1$ we associate with a formal scheme $\mathfrak{X}$ locally finitely presented over $k^{\circ}$ a $k$-analytic space $\mathfrak{X}_{\eta}$ (in the sense of [Ber1] and [Ber2]). In $\S 2$ we find that the morphism $\varphi_{\eta}: \mathfrak{Y}_{\eta} \rightarrow \mathfrak{X}_{\eta}$, which is induced by an étale morphism of formal schemes $\varphi: \mathfrak{Y} \rightarrow \mathfrak{X}$, possesses a certain property. Morphisms of $k$-analytic spaces with this property are called quasi-étale, and they give rise to a quasi-étale site $X_{\text {qét }}$ of a $k$ analytic space $X$. There is a canonical morphism of sites $X_{\text {qét }} \rightarrow X_{\text {et }}$, where $X_{\text {ét }}$ is the étale site introduced in [Ber2]. We show that the inverse image functor identifies the category of étale sheaves $X_{\text {êt }}$ with a full subcategory of $X_{\text {qét }}$ and preserves the cohomology groups. In $\S 4$ the quasi-étale topology is used to define, for a formal

* Incumbent of the Reiter Family Career Development Chair 
scheme $\mathfrak{X}$, a left exact functor $\Theta: \mathfrak{X}_{\eta_{\text {ét }}}^{\sim} \rightarrow \mathfrak{X}_{s \text { ét }}^{\sim}$ (the analog of the specialization functor $i^{*} j_{*}$ for schemes) and the vanishing cycles functor $\Psi_{\eta}: \mathfrak{X}_{\eta \text { ét }}^{\sim} \rightarrow \mathfrak{X}_{\bar{s} \text { ét. }}^{\sim}$. It is worthwhile to note that a spectral sequence connecting the étale cohomology of the generic fibre with that of the closed fibre exists for arbitrary formal schemes in contrast to the algebraic geometry situation when the similar spectral sequence exists only for proper schemes. At the end of $\S 4$ we prove that the formation of the vanishing cycles sheaves is compatible with extensions of the ground field. In $\$ 5$ we prove our first main result. It is a comparison theorem which states that if $\mathcal{X}$ is a scheme locally finitely presented over $k^{\circ}, \mathcal{F}$ is an étale abelian torsion sheaf on the generic fibre $\mathcal{X}_{\eta}$ of $\mathcal{X}$, and $\hat{\mathcal{F}}$ is the induced étale sheaf on the generic fibre $\hat{\mathcal{X}}_{\eta}$ of $\widehat{\mathcal{X}}$, then there are canonical isomorphisms $\dot{i}^{*}\left(R^{q} \hat{j}_{*} \mathcal{F}\right) \stackrel{\sim}{\rightarrow} R^{q} \Theta(\widehat{\mathcal{F}})$ and $R^{q} \Psi_{\eta}(\mathcal{F}) \stackrel{\sim}{\rightarrow} R^{q} \Psi_{\eta}(\widehat{\mathcal{F}})$. At the end of $\S 5$ we use this result to calculate the vanishing cycles sheaves for smooth formal schemes and to show that the cohomology groups for a certain class of compact $k$ analytic spaces are finite. In $\S 6$ we endow with a uniform space structure (see [Kel], Ch. 6) the sets of morphisms of analytic spaces $\operatorname{Mor}(Y, X)$. In a sense this structure depends uniformly on $X$. In $\$ 7$ we prove our second main result. Its particular case states the following. Let $X=\mathcal{M}(\mathcal{A})$ be a $k$-affinoid space, and let $f_{1}, \ldots, f_{n}$ be a $k$-affinoid generating system of elements of $\mathcal{A}$. Then for any discrete $\operatorname{Gal}\left(k^{s} / k\right)$ module $A$ and any element $\alpha \in H^{q}(X, \Lambda)$ there exist $t_{1}, \ldots, t_{n}>0$ such that, for any pair of morphisms $\varphi, \psi: Y \rightarrow X$ over $k$ with $\max _{y \in Y}\left|\left(\varphi^{*} f_{l}-\psi^{*} f_{i}\right)(y)\right| \leq t_{\imath}$, $1 \leq i \leq n$, one has $\varphi^{*}(\alpha)=\psi^{*}(\alpha)$ in $H^{q}(Y, \Lambda)$. The essential ingredient of the proof is a generalization of the classical Krasner's Lemma. The result implies, in particular, the following fact. If a $k$-analytic group $G$ acts on a $k$-analytic space $X$, then the étale cohomology groups of $X$ with compact support are discrete $G(k)$-modules. In $\S 8$ we apply the main result from $\S 7$ to the study of the action of the set of morphisms between the formal completions of schemes on their vanishing cycles sheaves.

In our paper [Ber3], we develop a formalism of vanishing cycles for nonArchimedean analytic spaces, which is an analog of the classical formalism over C from [SGA7], Exp. XIV, and we apply Theorem 7.1 of the present paper to establish results, similar to those established here, for the action of the set of morphisms between the formal completions of schemes (of finite type over an equicharacteristic Henselian discrete valuation ring) along closed points of the closed fibres on the stalks of the vanishing cycles sheaves at these points.

This work arose, on one hand, from a suggestion of $V$. Drinfeld to construct a vanishing cycles functor for formal schemes and, on the other hand, from a suggestion of P. Deligne to apply the étale cohomology theory for non-Archimedean analytic spaces developed in [Ber2] to his conjecture. I am very grateful to them for this. I would also like to thank V. Hinich for useful discussions.

\section{Analytic spaces associated with formal schemes}

Let $k$ be a non-Archimedean field, $k^{\circ}$ the ring of integers of $k, k^{\circ \circ}$ the maximal ideal of $k^{\circ}, \tilde{k}=k^{\circ} / k^{\circ}$ the residue field of $k$. If the valuation on $k$ is nontrivial, we fix a non-zero element $a \in k^{\circ}$. If the valuation on $k$ is trivial (then $\widetilde{k}=k^{\circ}=k$ and $k^{\circ \circ}=0$ ), we set $a=0$. Recall that the ring of restricted power series over $k^{\circ}$ in $n$ variables is the ring $k^{\circ}\{T\}=k^{\circ}\left\{T_{1}, \ldots, T_{n}\right\}$ of the formal power series $f=\sum_{\nu} a_{\nu} T^{\nu}$ over $k^{\circ}$ such that for any $m \geq 0$ the number of $\nu$ 's with $a^{n} \not a_{\nu}$ is 
finite. One has $k^{\circ}\{T\}=\lim k^{\circ} /\left(a^{m}\right)[T]$. (The ring $k^{\circ}\{T\}$ and the $a$-adic topology on it don't depend on the choice of a.) We remark that the Artin-Rees Lemma holds for any finitely generated ideal $\mathbf{a} \subset k^{\circ}\{T\}$, i.e., there exists $n_{0}$ such that $\mathbf{a} \cap a^{n} k^{\circ}\{T\} \subset$ $a^{n-n_{0}}$ a for all $n \geq n_{0}$. It follows that the quotient ring $k^{\circ}\{T\} / \mathbf{a}$ is separated and complete in the $a$-adic topology. A topologically finitely presented ring over $k^{\circ}$ is a ring of the form $k^{\circ}\{T\} / \mathbf{a}$ for some finitely generated ideal a $\subset k^{\circ}\{T\}$. We remark that if $A$ is such a ring, then the quotient ring $A / k^{\circ} A$ is finitely generated over the field $\widetilde{k}$. It follows that any open subset of the formal scheme $\operatorname{Spf}(A)$ is a finite union of open affine formal subschemes of the form $\operatorname{Spf}\left(A_{\{f\}}\right), f \in A$.

Let $k^{\circ}-\mathcal{F}$ sch denote the category of formal schemes locally finitely presented over $k^{\circ}$, i.e., the formal schemes over $\operatorname{Spf}\left(k^{\circ}\right)$ that are locally isomorphic to a formal scheme of the form $\operatorname{Spf}(A)$, where $A$ is topologically finitely presented over $k^{\circ}$. A formal scheme from $k^{\circ}-\mathcal{F} s c h$ which is a finite union of open affine formal subschemes of the above form is said to be finitely presented over $k^{\circ}$. (If the valuation on $k$ is trivial, then $k^{\circ}-\mathcal{F} s c h$ coincides with the category of schemes of locally finite type over $k$.) If $\mathfrak{X} \in k^{\circ}-\mathcal{F} s c h$, then the ringed space $\left(\mathfrak{X}, \mathcal{O}_{\mathfrak{X}} / k^{\circ \circ} \mathcal{O}_{\mathfrak{X}}\right)$ is a scheme of locally finite type over $\widetilde{k}$. It is called the closed fibre of $\mathfrak{X}$ and is denoted by $\mathfrak{X}_{s}$. We will define a functor $k^{\circ}-\mathcal{F}$ sch $\mapsto k-\mathcal{A} n$ that associates with a formal scheme $\mathfrak{X} \in k^{\circ}-\mathcal{F}$ sch its generic fibre $\mathfrak{X}_{\eta} \in k-\mathcal{A} n$, and we will construct a reduction map $\pi: \mathfrak{X}_{\eta} \rightarrow \mathfrak{X}_{s}$.

If $\mathfrak{X}=\operatorname{Spf}(A)$, where $A$ is topologically finitely presented over $k^{\circ}$, then $\mathcal{A}=$ $A \otimes_{k^{\circ}} k$ is a (strictly) $k$-affinoid algebra and $\mathfrak{X}_{\eta}$ is the $k$-affinoid space $\mathcal{M}(\mathcal{A})$. (It is clear that $\mathfrak{X} \mapsto \mathfrak{X}_{\eta}$ is a functor.) The image of $A$ in $\mathcal{A}$ is contained in $\mathcal{A}^{\circ}=$ $\{f \in \mathcal{A}|| f(x) \mid \leq 1$ for all $x \in \mathcal{M}(\mathcal{A})\}$, and therefore a point $x \in \mathfrak{X}_{\eta}$ gives rise to a character $\widetilde{\chi}_{x}: \tilde{A}:=A / k^{\circ 0} A \rightarrow \widetilde{\mathcal{H}(x)}$. The kernel of $\widetilde{\chi}_{x}$ (it is a prime ideal of $\widetilde{A})$ is, by definition, the point $\pi(x) \in \mathfrak{X}_{s}=\operatorname{Spec}(\tilde{A})$. The composition of $\pi$ with the canonical map $\mathfrak{X}_{s} \rightarrow \operatorname{Spec}(\tilde{\mathcal{A}})$ is the reduction map $\pi^{\prime}: \mathfrak{X}=\mathcal{M}(\mathcal{A}) \rightarrow \operatorname{Spec}(\tilde{\mathcal{A}})$ from [Ber1], $\S 2.4$. (Recall that $\widetilde{\mathcal{A}}=\mathcal{A}^{\circ} / \mathcal{A}^{\circ \circ}$, where $\mathcal{A}^{\circ \circ}=\{f \in \mathcal{A}|| f(x) \mid<1$ for all $x \in$ $\mathcal{M}(\mathcal{A})\}$.) By [Ber1], 2.4.4(i), the map $\pi^{\prime}$ is surjective. We take an epimorphism $k^{\circ}\{T\} \rightarrow A$. It induces epimorphisms $\tilde{k}[T] \rightarrow \widetilde{A}$ and $k\{T\} \rightarrow \mathcal{A}$. By [BGR], 6.3.5/1, the epimorphism $k\{T\} \rightarrow \mathcal{A}$ induces a finite homomorphism $\tilde{k}[T] \rightarrow \widetilde{\mathcal{A}}$. It is clear that the latter homomorphism coincides with the composition $\widetilde{k}[T] \rightarrow \widetilde{A} \rightarrow \widetilde{\mathcal{A}}$. It follows that the homomorphism $\widetilde{A} \rightarrow \widetilde{\mathcal{A}}$ is finite, and therefore the image of $\pi$ is a closed subset of $\mathfrak{X}_{s}$. Furthermore, if $\mathcal{Y}$ is a closed subset of $\mathfrak{X}_{s}$, then it is defined by an ideal $\left(\tilde{f}_{1}, \ldots, \tilde{f}_{n}\right)$ for some $f_{l} \in A$ and $\pi^{-1}(\mathcal{Y})=\left\{x \in \mathfrak{X}_{\eta}|| f_{l}(x) \mid<1,1 \leq i \leq n\right\}$. Let $\mathcal{Y}$ be an open subset of $\mathfrak{X}_{s}$, and let $\mathfrak{Y}$ be the open formal subscheme of $\mathfrak{X}$ with the underlying space $\mathcal{Y}$. If $\mathcal{Y}=\operatorname{Spec}\left(\widetilde{A}\left[\frac{1}{\tilde{f}}\right]\right)$ for some $f \in A$, then $\mathfrak{Y}=\operatorname{Spf}\left(A_{\{f\}}\right)$, $\pi^{-1}(\mathcal{Y})=\left\{x \in \mathfrak{X}_{\eta} \| f(x) \mid=1\right\}$ (it is a rational domain in $\mathfrak{X}_{\eta}$ ) and $\mathfrak{Y}_{\eta} \stackrel{\sim}{\rightarrow} \pi^{-1}(\mathcal{Y}$ ). It follows that $\pi^{-1}(\mathcal{Y})$ is always a closed analytic domain in $\mathfrak{X}_{\eta}$ for an arbitrary $\mathcal{Y}$. Suppose that the formal scheme $\mathfrak{Y}$ is affine, and let $\left\{\mathfrak{Y}_{\imath}\right\}_{\imath} \in I$ be a finite covering of $\mathfrak{Y}$ with $\mathfrak{Y}_{\imath}=\operatorname{Spf}\left(A_{\left\{f_{\imath}\right\}}\right), f_{\imath} \in A$. Then the canonical morphism $\mathfrak{Y} \rightarrow \mathfrak{X}$ identifies $\mathfrak{Y}_{\imath, \eta}$ with $\pi^{-1}\left(\mathcal{Y}_{2}\right)$, where $\mathcal{Y}_{1}=\operatorname{Spec}\left(\widetilde{A}\left[\frac{1}{\tilde{f}_{\imath}}\right]\right)$, and therefore, by Tate's Acyclicity Theorem, it identifies $\mathfrak{Y}_{\eta}$ with an affinoid domain in $\mathfrak{X}_{\eta}$ which evidently coincides with $\pi^{-1}(\mathcal{Y})$. 
If $\mathfrak{X}$ is arbitrary, we fix a locally finite covering $\left\{\mathfrak{X}_{\imath}\right\}_{\imath \in I}$ by open affine subschemes of the form $\operatorname{Spf}(A)$, where $A$ is topologically finitely presented over $k^{\circ}$. Suppose first that $\mathfrak{X}$ is separated. Then for any pair $i, j \in I$ the intersection $\mathfrak{X}_{\imath j}=\mathfrak{X}_{i} \cap \mathfrak{X}_{j}$ is also of the same form, $\mathfrak{X}_{\imath \jmath, \eta}$ is an affinoid domain in $\mathfrak{X}_{2, \eta}$, and the canonical morphism $\mathfrak{X}_{i j, \eta} \rightarrow \mathfrak{X}_{\imath, \eta} \times \mathfrak{X}_{j, \eta}$ is a closed immersion. By [Ber2], 1.3.3, we can glue all $\mathfrak{X}_{\imath, \eta}$ along $\mathfrak{X}_{\imath j, \eta}$, and we get a paracompact separated $k$-analytic space $\mathfrak{X}_{\eta}$. We remark that the correspondence $\mathfrak{X} \mapsto \mathfrak{X}_{\eta}$ is a functor that extends the functor constructed for the affine formal schemes, and if $\mathfrak{Y}$ is an open formal subscheme of $\mathfrak{X}$, then $\mathfrak{Y}_{\eta}$ is a closed analytic domain in $\mathfrak{X}_{\eta}$. Furthermore, the reduction maps $\mathfrak{X}_{\imath, \eta} \rightarrow \mathfrak{X}_{\imath, s}$ induce a reduction map $\pi: \mathfrak{X}_{\eta} \rightarrow \mathfrak{X}_{s}$. Finally, if $\mathfrak{X}$ is arbitrary, then $\mathfrak{X}_{\imath \jmath}=\mathfrak{X}_{\imath} \cap \mathfrak{X}_{j}$ are separated formal schemes, and $\mathfrak{X}_{\imath \jmath, \eta}$ is a compact analytic domain in the $k$-affinoid space $\mathfrak{X}_{\imath, \eta}$. Therefore we can glue all $\mathfrak{X}_{\imath, \eta}$ along $\mathfrak{X}_{\imath \jmath, \eta}$ and get a paracompact $k$ analytic space $\mathfrak{X}_{\eta}$. We remark that the correspondence $\mathfrak{X} \mapsto \mathfrak{X}_{\eta}$ is a functor to the category of paracompact strictly $k$-analytic spaces and this functor commutes with fibre products. We remark also that if $\mathfrak{X}$ is finitely presented, then $\mathfrak{X}_{\eta}$ is compact. The reduction maps $\mathfrak{X}_{\imath, \eta} \rightarrow \mathfrak{X}_{\imath, s}$ induce a reduction map $\pi: \mathfrak{X}_{\eta} \rightarrow \mathfrak{X}_{s}$. From the affine case it follows that the image of $\pi$ is a closed subset of $\mathfrak{X}_{s}$. Moreover, if $\mathcal{Y}$ is a closed subset of $\mathfrak{X}_{s}$, then $\pi^{-1}(\mathcal{Y})$ is an open subset of $\mathfrak{X}_{\eta}$. If $\mathcal{Y}$ is an open subset of $\mathfrak{X}_{s}$, then $\pi^{-1}(\mathcal{Y})$ is a closed analytic domain in $\mathfrak{X}_{\eta}$ and, if $\mathfrak{Y}$ is the open formal subscheme of $\mathfrak{X}$ with the underlying space $\mathcal{Y}$, then $\mathfrak{Y}_{\eta} \stackrel{\sim}{\rightarrow} \pi^{-1}(\mathcal{Y})$.

For a morphism $\varphi: \mathfrak{Y} \rightarrow \mathfrak{X}$ in $k^{\circ}-\mathcal{F} s c h$ we denote by $\varphi_{s}$ and $\varphi_{\eta}$ the induced morphisms $\mathfrak{Y}_{s} \rightarrow \mathfrak{X}_{s}$ and $\mathfrak{Y}_{\eta} \rightarrow \mathfrak{X}_{\eta}$, respectively. We remark that if $\varphi: \mathfrak{Y} \rightarrow \mathfrak{X}$ is finite (resp. flat finite), then the morphisms $\varphi_{s}$ and $\varphi_{\eta}$ are also finite (resp. flat finite).

\section{2. Étale morphisms of formal schemes}

Let $\mathfrak{X} \in k^{\circ}-\mathcal{F} s c h$. For $n \geq 1$, let $\mathfrak{X}_{n}$ denote the scheme $\left(\mathfrak{X}, \mathcal{O}_{\mathfrak{X}} / a^{n} \mathcal{O}_{\mathfrak{X}}\right)$. (It is a scheme locally finitely presented over $k^{\circ} /\left(a^{n}\right)$.) A morphism of formal schemes over $k^{\circ}, \varphi: \mathfrak{Y} \rightarrow \mathfrak{X}$, is said to be étale if $\mathfrak{Y} \in k^{\circ}-\mathcal{F}$ sch and for all $n \geq 1$ the induced morphisms of schemes $\varphi_{n}: \mathfrak{Y}_{n} \rightarrow \mathfrak{X}_{n}$ are étale. The following two lemmas are consequences of the local description of étale morphisms of schemes.

Lemma 2.1. Let $\mathfrak{X} \in k^{\circ}-\mathcal{F}_{\text {sch }}$. Then the correspondence $\mathfrak{Y} \mapsto \mathfrak{Y}_{s}$ induces an equivalence between the category of formal schemes étale over $\mathfrak{X}$ and the category of schemes étale over $\mathfrak{X}_{s}$.

Proof. It is clear that the functor is fully faithful. Therefore to show that it is essentially surjective, it suffices to construct a lifting of an étale morphism $\mathcal{Y} \rightarrow \mathfrak{X}_{s}$ locally. Thus, we may assume that $\mathfrak{X}=\operatorname{Spf}(A)$, where $A$ is topologically finitely presented over $k^{\circ}$, and, by the local description of étale morphisms of schemes, that $\mathcal{Y}=\operatorname{Spec}\left(C_{-}\right)$, where $C=\widetilde{A}[T] /(\widetilde{P}), \widetilde{P}$ is a monic polynomial, $\widetilde{f}$ is an element of $C$ such that the image of the derivative $\widetilde{P}^{\prime}$ in $C_{\widetilde{f}}$ is invertible. Let $P$ be a monic polynomial in $A[T]$ whose image in $\widetilde{A}[T]$ is $\widetilde{P}$. Then $B:=A[T] /(P) \stackrel{\sim}{\rightarrow} A\{T\} /(P)$, i.e., $B$ is topologically finitely presented over $k^{\circ}$ and is free of finite rank over $A$, and $\widetilde{B} \stackrel{\sim}{\rightarrow} C$. Furthermore, let $f$ be an element of $B$ whose image in $\widetilde{B}$ is $\tilde{f}$, and let $\mathfrak{Y}=\operatorname{Spf}\left(B_{\{f\}}\right)$. Since $\widetilde{B_{\{f\}}} \stackrel{\sim}{\rightarrow} \widetilde{B}_{\tilde{f}}$, then $\mathcal{Y} \stackrel{\sim}{\rightarrow} \mathfrak{Y}_{s}$. Moreover, since $B_{\{f\}} / a^{n} B_{\{f\}} \stackrel{\sim}{\rightarrow}\left(B / a^{n} B\right)_{f}$, then the 
image of the derivative $P^{\prime}$ in $B_{\{f\}} / a^{n} B_{\{f\}}$ is invertible, and therefore the morphism $\mathfrak{Y}_{n} \rightarrow \mathfrak{X}_{n}$ is étale. It follows that the morphism $\mathfrak{Y} \rightarrow \mathfrak{X}$ is étale.

The statement of Lemma 2.1 for the class of finite étale morphisms follows from [SGA1], Exp. I, 8.4. (Of course, the above proof uses the argument from [SGA1].)

Lemma 2.2. Let $\varphi: \mathfrak{Y} \rightarrow \mathfrak{X}$ be an étale morphism. Then $\varphi_{\eta}\left(\mathfrak{Y}_{\eta}\right)=\pi^{-1}\left(\varphi_{s}\left(\mathfrak{Y}_{s}\right)\right)$. In particular, $\varphi_{\eta}\left(\mathfrak{Y}_{\eta}\right)$ is a closed analytic domain in $\mathfrak{X}_{\eta_{\eta}}$.

Proof. Since the statement is true when $\mathfrak{Y}$ is an open formal subscheme of $\mathfrak{X}$, we can shrink $\mathfrak{X}$ and $\mathfrak{Y}$ and assume that $\mathfrak{X}=\operatorname{Spf}(A)$, where $A$ is topologically finitely presented over $k^{\circ}$, and $\mathfrak{Y}=\operatorname{Spf}\left(B_{\{f\}}\right)$, where $B$ is a finite free $A$-module and $f \in B$. Let $T^{n}+g_{1} T^{n-1}+\ldots+g_{n}$ be the characteristic polynomial of $f$ over $A$. It is easy to see that $\varphi_{s}\left(\mathfrak{Y}_{s}\right)=\cup_{\imath=1}^{n}\left\{\mathbf{x} \in \mathfrak{X}_{s} \mid g_{z}(\mathbf{x}) \neq 0\right\}$ and $\varphi_{\eta}\left(\mathfrak{Y}_{\eta}\right)=\cup_{i=1}^{n}\left\{x \in \mathfrak{X}_{\eta}|| g_{z}(x) \mid=1\right\}$. This gives the required equality.

Proposition 2.3. Let $\varphi: \mathfrak{Y} \rightarrow \mathfrak{X}$ be an étale morphism. Then for every point $y \in \mathfrak{Y}_{\eta}$ there exist affinoid domains $V_{1}, \ldots, V_{n} \subset \mathfrak{Y}_{\eta}$ such that $V_{1} \cup \ldots \cup V_{n}$ is a neighborhood of $y$ and each $V_{2}$ can be identified with an affinoid domain in a $k$-analytic space étale over $\mathfrak{X}_{\eta}$.

Proof. Consider first the case when $\varphi$ is of the form $\mathfrak{Y}=\operatorname{Spf}\left(B_{\{f\}}\right) \rightarrow \mathfrak{X}=\operatorname{Spf}(A)$, where $B=A[T] /(P) \stackrel{\sim}{\rightarrow} A\{T\} /(P), P$ is a monic polynomial and $f$ is an element of $B$ such that the image of $P^{\prime}$ in $\widetilde{B}_{\tilde{f}}=\left(\widetilde{A}[T] /(\widetilde{P})_{r_{f}}\right.$ is invertible. If $g$ is the image of $P^{\prime}$ in $B$, then the latter implies that $|g(z)|=1$ for all $z \in \mathfrak{Y}_{\eta}$. Furthermore, the formal scheme $\mathfrak{Z}=\operatorname{Spf}(B)$ is finite flat over $\mathfrak{X}$, and the $k$-analytic space $\mathfrak{Z}_{\eta}$ is finite flat over $\mathfrak{X}_{\eta}$. One has $\mathfrak{Y}_{\eta}=\left\{z \in \mathfrak{Z}_{\eta}|| f(z) \mid=1\right\}$. The morphism $\mathfrak{Z}_{\eta} \rightarrow \mathfrak{X}_{\eta}$ is étale at a point $z \in \mathfrak{Z}_{\eta}$ if and only if $g(z) \neq 0$. Since $|g(z)|=1$ for all $z \in \mathfrak{Y}_{\eta}$, then $\mathfrak{Y}_{\eta} \subset\left\{z \in \mathfrak{Z}_{\eta} \mid g(z) \neq 0\right\}$, i.e., $\mathfrak{Y}_{\eta}$ can be identified with an affinoid domain in a $k$-analytic space étale over $\mathfrak{X}_{\eta}$.

Consider now the general case. We can find open affine formal subschemes $\mathfrak{X}_{1}, \ldots, \mathfrak{X}_{n} \subset \mathfrak{X}$ and $\mathfrak{Y}_{1}, \ldots, \mathfrak{Y}_{n} \subset \mathfrak{Y}$ such that $y \in \mathfrak{Y}_{1, \eta} \cap \ldots \cap \mathfrak{Y}_{n, \eta}, \mathfrak{Y}_{1, \eta} \cup$ $\ldots \cup \mathfrak{Y}_{n, \eta}$ is a neighborhood of $y$ and $\varphi$ induces étale morphisms $\mathfrak{Y}_{\imath} \rightarrow \mathfrak{X}_{\imath}$ of the above form. By the first case, each $\mathfrak{Y}_{2, \eta}$ can be identified with an affinoid domain in a $k$-analytic space étale over $\mathfrak{X}_{1, \eta}$. From [Ber2], 3.4.2, it follows that we can find, for each $\mathrm{i}$, an affinoid neighborhood $V_{2}$ of $y$ in $\mathfrak{Y}_{7, \eta}$ such that $V_{2}$ can be identified with an affinoid domain in a $k$-analytic space étale over $\mathfrak{X}_{\eta}$. Since $V_{1} \cup \ldots \cup V_{t_{\mathrm{b}}}$ is a neighborhood of $y$ in $\mathfrak{Y}_{\eta}$, the required statement follows.

\section{Quasi-étale topology on an analytic space}

Let $\varphi: Y \rightarrow X$ be a morphism of $k$-analytic spaces. We say that $\varphi$ is quasi-étale if for every point $y \in Y$ there exist affinoid domains $V_{1}, \ldots, V_{n} \subset Y$ such that $V_{1} \cup \ldots \cup V_{n}$ is a neighborhood of $y$ and each $V_{\imath}$ can be identified with an affinoid domain in a $k$-analytic space étale over $X$. For example, étale morphisms and the canonical embeddings of analytic domains in a $k$-analytic space are quasi-étale. Furthermore, by Proposition 2.3, if $\mathfrak{Y} \rightarrow \mathfrak{X}$ is an étale morphism of formal schemes in $k^{\circ}-\mathcal{F}_{s c h}$, then the induced morphism of their generic fibres $\mathfrak{Y}_{\eta} \rightarrow \mathfrak{X}_{\eta}$ is quasi-étale. We remark 
that if a morphism $\varphi: Y \rightarrow X$ is quasi-étale, then for every point $y \in Y$ there exist affinoid domains $V_{1}, \ldots, V_{n} \subset Y$ such that $V_{1} \cup \ldots \cup V_{n}$ is a neighborhood of $y$ and each $V_{\imath}$ can be identified over $X$ with an affinoid domain in a paracompact $k$-analytic space separated and étale over $X$.

Lemma 3.1. (i) Quasi-étale morphisms are preserved under compositions, under any base change functor and under any ground field extension functor. étale.

(ii) If $Y$ and $Z$ are quasi-étale over $X$, then any $X$-morphism $Z \rightarrow Y$ is quasi-

Proof. All the statements easily follow from the corresponding properties of étale morphisms and Theorem 3.4.1 from [Ber2].

For a $k$-analytic space $X$, let Qét $(X)$ denote the category of quasi-étale morphisms $U \rightarrow X$. The quasi-étale topology on $X$ is the Grothendieck topology on the category Qét $(X)$ generated by the pretopology for which the set of coverings of $(U \rightarrow X) \in$ Qét $(X)$ is formed by the families $\left\{U_{2} \stackrel{f_{2}}{\longrightarrow} U\right\}_{2 \in I}$ such that each point of $U$ has a neighborhood of the form $f_{i_{1}}\left(V_{1}\right) \cup \ldots \cup f_{\imath_{n}}\left(V_{n}\right)$ for some affinoid domains $V_{1} \subset$ $U_{\imath_{1}}, \ldots, V_{n} \subset U_{n_{n}}$. (It is easy to verify that the latter really defines a pretopology on Qét $(X)$.) We denote by $X_{\text {quet }}$ the site obtained in this way (the quasi-étale site of $X$ ) and by $X_{\text {qét }}$ the category of sheaves of sets on $X_{\text {qét }}$ (the quasi-étale topos of $X$ ). It is clear that there is a morphism of sites $\mu: X_{\text {qét }} \rightarrow X_{\text {ét }}$. We remark that there is also a morphism of sites $X_{\text {qét }} \rightarrow X_{G}$, where $X_{G}$ is defined in [Ber2], $\delta 1.3$. We are going to establish the relationship between the topoi $X_{\text {qét }}^{\sim}$ and $X_{\text {ét }}^{\sim}$ similar to that between the topoi $X_{G}^{\sim}$ and $X^{\sim}$ established in [Ber2].

Recall ([God], §II.3.4) that a sheaf $F$ on a topological space $T$ is called soft if for any closed subset $\Sigma \subset T$ the map $F(T) \rightarrow F(\Sigma)$ is surjective. If $T$ is locally compact and paracompact, then $F$ is soft if and only if the above map is surjective for any compact subset $\Sigma \subset T$. We say that an étale abelian sheaf $F$ on a $k$-analytic space $X$ is soft if, for each point $x \in X, F_{x}$ is a flabby $G_{\mathcal{H}(x)}$-module and, for each paracompact $U$ étale over $X$, the restriction of $F$ to the usual topology $|U|$ of $U$ is a soft sheaf. For example, any injective sheaf on $X$ is soft ([Ber2], 4.2.5).

Lemma 3.2. Let $F$ be an étale abelian soft sheaf on a $k$-analytic space $X$. Then

(i) if $X$ is paracompact, then $H^{q}(X, F)=0$ for $q \geq 1$;

(ii) if $\varphi: Y \rightarrow X$ is a morphism of analytic spaces over $k$, then the sheaf $\varphi^{*} F$ is soft in any of the following cases:

(a) $\varphi$ is a quasi-étale morphism;

(b) $\varphi$ is a quasi-immersion (see [Ber2], \$4.3);

(c) $\varphi$ is the canonical morphism $\bar{X}=X \widehat{\otimes} \widehat{k}^{a} \rightarrow X$.

Here $k^{a}$ denotes the algebraic closure of $k$. If the valuation on $k$ is nontrivial, then the separable closure $k^{s}$ of $k$ is everywhere dense in $k^{a}$ (i.e., $\widehat{k^{s}}=\widehat{k^{a}}$ ).

Proof (i) Consider the spectral sequence $E_{2}^{p, q}=H^{p}\left(|X|, R^{q} \pi_{*} F\right) \Rightarrow H^{p+q}(X, F)$ of the morphism of sites $\pi: X_{\text {et }} \rightarrow|X|$ (see [Ber2], \$4.2). By [Ber2], 4.2.4, for a point $x \in X$ one has $\left(R^{q} \pi_{*} F\right)_{x}=H^{q}\left(G_{\mathcal{H}(x)}, F_{x}\right)$. It follows that $R^{q} \pi_{*} F=0$ for $q \geq 1$, and therefore $H^{q}(X, F)=H^{q}\left(|X|, \pi_{*} F\right)$. The latter group is equal to zero for $q \geq 1$ because $\pi_{*} F$ is a soft sheaf on the paracompact space $X$.

(ii) First of all, if $y \in Y$ and $x=\varphi(y)$, then in all the cases $G_{\mathcal{H}(y)}$ is a closed

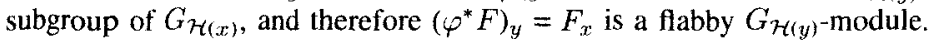


To verify the statement in the cases (a) and (b), it suffices to show that the restriction of $\varphi^{*} F$ to $|Y|$ is a soft sheaf when $\varphi: Y \rightarrow X$ is a morphism of analytic spaces over $k, Y$ is paracompact and each point $y \in Y$ has a neighborhood of the form $V_{1} \cup \ldots \cup V_{n}$, where $V_{\imath}$ are affinoid domains in $Y$, such that for each $i$ there exists a quasi-immersion of $V_{t}$ in a paracompact $k$-analytic space $\mathcal{U}_{2}$ étale over $X$. Since the property of a sheaf to be soft is a local one ([God], 11.3.4.1), we may assume that $Y=V_{1} \cup \ldots \cup V_{n}$, where $V_{\imath}$ are as above. The restriction of $F$ to $\left|\mathcal{U}_{n}\right|$ is a soft sheaf. Since $\left|V_{\imath}\right|$ is a closed subset of $\left|\mathcal{U}_{2}\right|$, then, by [God], II.3.4.2, the restriction of $\varphi^{*} F$ to $\left|V_{2}\right|$ is a soft sheaf. It follows that the restriction of $\varphi^{*} F$ to $|Y|$ is a soft sheaf.

Let $Z \rightarrow \bar{X}$ be an étale morphism. Since the property of a sheaf to be soft is local and $Z$ can be defined locally over a finite separable extension of $k$, we may assume that $Z=\bar{Y}$, where $Y$ is a paracompact $k$-analytic space étale over $X$. Since the space $\bar{Y}$ is locally compact, it suffices to verify that for any compact subset $\Sigma \subset \bar{Y}$ the map $F(\bar{Y}) \rightarrow F(\Sigma)$ is surjective. This is established in the proof of Corollary 5.3 .5 from [Ber2].

Theorem 3.3. Let $f: U \rightarrow X$ be a quasi-étale morphism, and $F$ an étale sheaf on $X$. Then

(i) $\left(f^{*} F\right)(U) \stackrel{\sim}{\rightarrow}\left(\mu^{*} F\right)(U)$, where $f^{*}$ is the inverse image functor $X_{\text {ét }} \rightarrow U_{\text {ét }}^{\sim}$;

(ii) if $F$ is an abelian sheaf, then $H^{q}\left(U, f^{*} F\right) \stackrel{\sim}{\rightarrow} H^{q}\left(U_{\text {qét }}, \mu^{*} F\right)$ for all $q \geq 0$.

Proof . (i) The map $\left(f^{*} F\right)(U) \rightarrow\left(\mu^{*} F\right)(U)$ is a composition of the evident maps

$$
\left(f^{*} F\right)(U) \rightarrow\left(\mu_{Y}^{*} f^{*} F\right)(U) \stackrel{\sim}{\rightarrow}\left(f_{\text {quét }}^{*} \mu^{*} F\right)(U)=\left(\mu^{*} F\right)(U) .
$$

where $\mu_{Y}$ and $f_{\text {qé }}$ are the morphisms of sites $Y_{\text {qét }} \rightarrow Y_{\text {ét }}$ and $Y_{\text {qét }} \rightarrow X_{\text {qét }}$, respectively. Thus, to prove (i), it suffices to verify the following two facts:

(1) the presheaf $U \mapsto\left(f^{*} F\right)(U)$ is a sheaf on $X_{\text {qét }}$;

(2) for any $(U \stackrel{f}{\longrightarrow} X) \in \operatorname{Qét}(X)$, there is a covering $\left\{U_{2} \stackrel{g_{2}}{\longrightarrow} U\right\}_{\imath \in 1}$ in $X_{\text {qét }}$ such that $\left.\left(\left(f g_{\imath}\right)^{*} F\right)\left(U_{\imath}\right) \stackrel{\sim}{\rightarrow}\left(\mu^{*} F\right)\left(U_{\imath}\right)\right)$ for all $i \in I$.

(1) Let $\left\{U_{2} \stackrel{g_{2}}{\longrightarrow} U\right\}_{2 \in I}$ be a covering in $X_{\text {qét }}$. We have to verify that there is an exact sequence

$$
\left(f^{*} F\right)(U) \longrightarrow \prod_{\imath}\left(f_{i}^{*} F\right)\left(U_{\imath}\right) \longrightarrow \prod_{i, j}\left(f_{i, j}^{*} F\right)\left(U_{\imath \jmath}\right)
$$

where $f_{\imath}$ and $f_{\imath \jmath}$ are the morphisms $U_{1} \rightarrow U \rightarrow X$ and $U_{\imath \jmath}:=U_{\imath} \times_{U} U_{\jmath} \rightarrow U \rightarrow X$, respectively. Consider first the case when $U$ is $k$-affinoid. In this case we can replace the covering by a finite refinement and assume that each $U_{2}$ is $k$-affinoid and can be identified with an affinoid domain in a $k$-analytic space $V_{\imath}$ separated and étale over $U$. If $\mathcal{U}_{2}$ are arbitrary open neighborhoods of $U_{2}$ in $V_{\imath}$, then $\left\{\mathcal{U}_{\imath} \longrightarrow U\right\}_{\imath} \in I$ is a finite étale covering of $U$, and therefore there is an exact sequence

$$
\left(f^{*} F\right)(U) \longrightarrow \prod_{i}\left(f^{*} F\right)\left(\mathcal{U}_{i}\right) \Longrightarrow \prod_{\imath, j}\left(f^{*} F\right)\left(\mathcal{U}_{\imath j}\right)
$$

where $\mathcal{U}_{\imath \jmath}=\mathcal{U}_{2} \times_{U} \mathcal{U}_{\jmath}$. By [Ber2], 44.3 , one has $\left(f_{\imath}^{*} F\right)\left(U_{\imath}\right)=\lim \left(f^{*} F\right)\left(\mathcal{U}_{\imath}\right)$ and $\left(f_{1 j}^{*} F\right)\left(U_{\imath \jmath}\right)=\lim \left(f^{*} F\right)\left(\mathcal{U}_{2 j}\right)$ when all $\mathcal{U}_{\imath}$ tend to $U_{\imath}$. Hence, the sequence $(*)$ being a filtered inductive limit of the exact sequences $(* *)$ is exact. 
By the first case and the fact that a basis of topology of a $k$-analytic space is formed by open paracompact sets, it remains to verify the exactness of $(*)$ when $U$ is paracompact and $\left\{U_{2}\right\}_{\imath \in I}$ is a locally finite covering of $U$ by affinoid domains. In this case the exactness of (*) follows from [Ber2], §4.3, and [God], II.1.3.1.

Lemma 3.4. Let $\varphi: Y \rightarrow X$ be an étale morphism with Hausdorff $X$, and let $\Sigma$ be a compact subset of $Y$. Suppose that $\varphi$ is injective on $\Sigma$ and, for each point $y \in \Sigma$, one has $\mathcal{H}(\varphi(y)) \stackrel{\sim}{\rightarrow} \mathcal{H}(y)$. Then there is an open neighborhood $\mathcal{V}$ of $\Sigma$ such that $\varphi$ induces an isomorphism $\mathcal{V} \stackrel{\sim}{\longrightarrow} \varphi(\mathcal{V})$.

Proof. By Theorem 3.4.1 from [Ber2], $\varphi$ is a local isomorphism at every point $y \in \Sigma$. Therefore we can shrink $Y$ and assume that $\varphi$ is a local isomorphism at all points $Y$. In particular, it suffices to find an open neighborhood $\mathcal{V}$ of $\Sigma$ such that $\varphi$ is injective on $\mathcal{V}$. One has $\Sigma \subset \mathcal{V}_{1} \cup \ldots \cup \mathcal{V}_{n}$, where $\mathcal{V}_{2}$ and $\varphi\left(\mathcal{V}_{2}\right)$ are open and $\mathcal{V}_{\imath} \stackrel{\sim}{\rightarrow} \varphi\left(\mathcal{V}_{\imath}\right)$. By induction, to construct $V$ it suffices to consider the case $n=2$. Since $X$ is Hausdorff, then the image of $X$ in $X \times X$ is closed. It follows that the image of $Y \times{ }_{X} Y$ in $Y \times Y$ is closed. Furthermore, since the map $|Y \times Y| \rightarrow|Y| \times|Y|$ is compact, then the image of $\left|Y \times_{X} Y\right|$ in $|Y| \times|Y|$, that coincides with $|Y| \times_{|X|}|Y|$, is closed. It follows that one can find open neighborhoods $\Sigma \backslash \mathcal{V}_{2} \subset \mathcal{W}_{1} \subset \mathcal{V}_{1}$ and $\Sigma \backslash \mathcal{V}_{1} \subset \mathcal{W}_{2} \subset \mathcal{V}_{2}$ such that $\left|\mathcal{W}_{1}\right| \times\left|\mathcal{W}_{2}\right|$ has empty intersection with $|Y| \times_{|X|}|Y|$. The open set $\mathcal{V}=\mathcal{W}_{1} \cup \mathcal{W}_{2} \cup\left(\mathcal{V}_{1} \cap \mathcal{V}_{2}\right)$ contains $\Sigma$ and $\varphi$ is injective on $\mathcal{V}$.

(2) We remark that the sheaf $\mu^{*} F$ is associated with the presheaf $\mu^{p} F$ for which $\left(\mu^{p} F\right)(U)=\lim F(Y)$, where the limit is taken over all morphisms over $X$ from $U$

to $k$-analytic spaces étale over $X$. Let $(U \stackrel{f}{\longrightarrow} X) \in$ Qét( $X)$. To prove (2), it siffices to show that $\left(f^{*} F\right)(U) \stackrel{\sim}{\rightarrow}\left(\mu^{p} F\right)(U)$ under the assumption that $U$ is $k$-affinoid and is identified with an affinoid domain in a Hausdorff $k$-analytic space $V$ étale over $X$. We know that $\left(f^{*} F\right)(U)=\lim F(\mathcal{V})$, where $\mathcal{V}$ runs through open neighborhoods of $U$ in $V$. It suffices to show that any morphism over $X$ from $U$ to $Y$, which is étale over $X$, extends to a morphism over $X$ from an open neighborhood of $U$ in $V$ to $Y$. For this we remark that the morphism $U \rightarrow Y \times{ }_{X} V \stackrel{p r}{\longrightarrow} V$ satisfies the conditions of Lemma 3.4. Therefore there exists an open neighborhood $\mathcal{W}$ of $U$ in $Y \times_{X} V$ for which $\mathcal{W} \stackrel{\sim}{\rightarrow} \mathcal{V}:=\operatorname{pr}(\mathcal{W})$. It follows that the morphism $U \rightarrow Y$ extends to a morphism $\mathcal{V} \rightarrow Y$ over $X$.

(ii) Since an open covering of $U$ is a covering in the étale and the quasi-étale topologies, then the Leray spectral sequences generated by it in the both topologies show that it suffices to prove the statement for sufficiently small $U$. In particular, we may assume that $U$ is paracompact. Furthermore, consider a locally finite covering of $U$ by affinoid domains. It is a covering of $U$ in the quasi-étale topology, and therefore it induces a Leray spectral sequence which is convergent to $H^{*}\left(U_{\text {qét }}, \mu^{*} F\right)$. By [Ber2], 4.3.7, it induces also a similar Leray spectral sequence which is convergent to $H^{*}\left(U, f^{*} F\right)$. Therefore it suffices to prove the statement when $U$ is $k$-affinoid and can be identified with an affinoid domain in a paracompact $k$-analytic space $V$ étale over $X$. Finally, by (i), the statement is true for $q=0$. Therefore, by Lemma 3.2, it suffices to show that if $F$ is injective, then $H^{q}\left(U_{\text {gét }}, \mu^{*} F\right)=0$ for all $q \geq 1$. We remark that any quasi-étale covering of $U$ can be refined to a finite covering of the form $\mathcal{U}=\left\{U_{\imath} \longrightarrow U\right\}_{\imath \in I}$, where each $U_{\imath}$ is $k$-affinoid and can be identified with an affinoid domain in a paracompact $k$-analytic space $V_{i}$ étale over $X$. It follows that it 
suffices to show that the Čech cohomology groups $\breve{H}^{q}\left(\mathcal{U}, \mu^{*} F\right)$ associated with such a covering are equal to zero for all $q \geq 1$. These groups are the cohomology groups of the Čech complex $C\left(\mathcal{U}, \mu^{*} F\right)$ associated with $\mathcal{U}$. If $\mathcal{V}_{\imath}$ are open neighborhoods of $U_{\imath}$ in $V_{\imath}$, then shrinking $V$ we may assume that $\mathcal{V}=\left\{\mathcal{V}_{\imath} \longrightarrow V\right\}_{\imath \in I}$ is an étale covering of $V$. The sheaf $\left.F\right|_{V}$ is injective, and therefore the Čech complex $C(\mathcal{V}, F)$ associated with the covering $\mathcal{V}$ is exact. Since the complex $C\left(\mathcal{U}, \mu^{*} F\right)$ is a filtered inductive limit of the complexes $C(\mathcal{V}, F)$ (when all $\mathcal{U}_{2}$ tend to $U_{2}$ ), it follows that the complex $C \cdot\left(\mathcal{U}, \mu^{*} F\right)$ is exact. The theorem is proved.

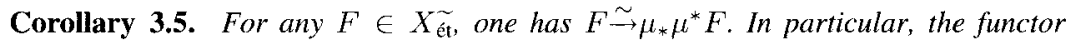
$\mu^{*}: X_{\text {êt }}^{\sim} \rightarrow X_{\text {qết }}^{\sim}$ is fully faithful.

For a morphism $f: Y \rightarrow X$ and an étale (resp. étale abelian) sheaf $F$ on $X$ we use the notation $F(Y)$ (resp. $H^{q}(Y, F)$ ) instead of $\left(f^{*} F\right)(Y)$ (resp. $H^{q}\left(Y, f^{*} F\right)$ ). The following is a generalization of the Leray spectral sequence 4.3.7 from [Ber2] (which was used in the proof of the Theorem 3.3).

Corollary 3.6. For an étale abelian sheaf $F$ on $X$ and $q \geq 0$, let $\mathcal{H}^{q}(F)$ denote the presheaf $V \mapsto H^{q}(V, F)$ on $X_{\text {qèt. }}$. Then for any quasi-étale covering $\mathcal{V}=\left\{V_{\imath} \rightarrow X\right\}_{\imath \in I}$ there is a spectral sequence $E_{2}^{p, q}=\check{H}^{p}\left(\mathcal{V}, \mathcal{H}^{q}(F)\right) \Longrightarrow H^{p+q}(X, F)$.

The quasi-étale cohomology groups of a quasi-étale abelian sheaf $F$ will be denoted by $H^{q}(X, F)$. Due to Theorem 3.3, this is consistent with the notation of étale cohomology groups if $F$ comes from an étale sheaf.

Corollary 3.7. Let $\varphi: Y \rightarrow X$ be a compact morphism, and let $F$ be an étale (resp. étale abelian) sheaf on $Y$. Then $\mu^{*}\left(\varphi_{*} F\right) \stackrel{\sim}{\rightarrow} \varphi_{*}\left(\mu^{*} F\right)\left(\right.$ resp. $\mu^{*}\left(R^{q} \varphi_{*} F\right) \stackrel{\sim}{\rightarrow} R^{q} \varphi_{*}\left(\mu^{*} F\right)$, $q \geq 0$ ).

Proof Let $f: U \rightarrow X$ be a quasi-étale morphism. By Theorem 3.3(i), $\left(\mu^{*} \varphi_{*} F\right)(U)$ $=\left(f^{*} \varphi_{*} F\right)(U)$. Since $\varphi$ is compact, then $\left(f^{*} \varphi_{*} F\right)(U) \stackrel{\sim}{\rightarrow}\left(\varphi_{*}^{\prime} f^{\prime *} F\right)(U)$, where $\varphi^{\prime}$ and $f^{\prime}$ are the induced morphisms $Y \times_{X} U \rightarrow U$ and $Y \times_{X} U \rightarrow Y$. Therefore, we get $\left(\mu^{*} \varphi_{*} F\right)(U) \stackrel{\sim}{\rightarrow}\left(f^{*} F\right)\left(Y \times_{X} U\right) \stackrel{\sim}{\rightarrow}\left(\mu^{*} F\right)\left(Y \times_{X} U\right)=\left(\varphi_{*} \mu^{*} F\right)(U)$. To prove the statement, it suffices to verify that if $F$ is abelian injective, then $R^{q} \varphi_{*}\left(\mu^{*} F\right)=0$ for $q \geq 1$. This follows from Theorem 3.3(ii).

We note that the above statement is not true without the assumption that the morphism is compact. Indeed, let $j$ be the open immersion $D=D(0,1) \hookrightarrow E=$ $E(0,1)$ and let $F$ be a non-zero constant sheaf on $D$. Then the restriction of $\mu^{*}\left(j_{*} F\right)$ to the annulus $A=E \backslash D$ is a non-zero sheaf but the restriction of $j_{*}\left(\mu^{*} F\right)$ to $A$ is zero.

\section{The vanishing cycles functor}

Let $\mathfrak{X} \in k^{\circ}-\mathcal{F} s c h$. We fix a functor $\mathfrak{Y}_{s} \mapsto \mathfrak{Y}$ from the category of schemes étale over $\mathfrak{X}_{s}$ to the category of formal schemes étale over $\mathfrak{X}$ which is inverse to the functor from Lemma 2.1. By Lemma 2.2 and Proposition 2.3, the composition of the functor $\mathfrak{Y}_{s} \mapsto \mathfrak{Y}$ with the functor $\mathfrak{Y} \mapsto \mathfrak{Y}_{\eta}$ induces a morphism of sites $\nu: \mathfrak{X}_{\eta_{\text {ét }}} \rightarrow \mathfrak{X}_{s \hat{s e t}}$. We get a left exact functor

$$
\Theta=\nu_{*} \mu^{*}: \mathfrak{X}_{\eta_{\text {ét }}^{\sim}}^{\sim} \longrightarrow \mathfrak{X}_{\eta_{\text {qét }}^{\sim}} \longrightarrow \mathfrak{X}_{s e ̂ t}^{\sim} .
$$


(The similar functor for a bigger field $K$ will be denoted by $\Theta_{K}$.)

Proposition 4.1. Let $F$ be an étale sheaf on $\mathfrak{X}_{\eta}$.

(i) If $\mathfrak{Y}_{s}$ is étale over $\mathfrak{X}_{s}$, then $\Theta(F)\left(\mathfrak{Y}_{s}\right)=F\left(\mathfrak{Y}_{\eta}\right)$.

(ii) If $F$ is an abelian sheaf, then $R^{q} \Theta(F)$ is associated with the presheaf $\mathfrak{Y}_{s} \mapsto$ $H^{q}\left(\mathfrak{Y}_{\eta}, F\right)$.

(iii) If $F$ is a soft abelian sheaf, then the sheaf $\Theta(F)$ is flabby.

Proof . (i) and (ii) follow directly from Lemma 3.2 and Theorem 3.3. To prove (iii), it suffices to verify that the Cech cohomology groups $\breve{H}^{q}\left(\mathfrak{Y}_{s}, \theta(F)\right)$ of an étale covering $\mathfrak{V}_{s}=\left\{\mathfrak{Y}_{\imath, s} \longrightarrow \mathfrak{Y}_{s}\right\}_{s \in I}$ in $\mathfrak{X}_{s e ́ t}^{\sim}$ are trivial for $q \geq 1$. By (i), these groups coincide with the Čech cohomology groups $\breve{H}^{q}\left(\mathfrak{V}_{\eta}, F\right)$, where $\mathfrak{V}_{\eta}=\left\{\mathfrak{Y}_{\imath, \eta} \longrightarrow \mathfrak{Y}_{\eta}\right\}_{\imath \in I}$ is the quasi-étale covering of $\mathfrak{Y}_{\eta}$ induced by $\mathfrak{V}_{s}$. From Corollary 3.6 and Lemma 3.2 it follows that $\breve{H}^{q}\left(\mathfrak{V}_{\eta}, F\right) \stackrel{\sim}{\rightarrow} H^{q}\left(\mathfrak{Y}_{\eta}, F\right)$. The latter group is trivial for $q \geq 1$, by the same Lemma 3.2 .

Corollary 4.2. (i) For an étale morphism $\mathfrak{Y} \rightarrow \mathfrak{X}$ in $k^{\circ}-\mathcal{F}$ sch and $F \in \mathbf{S}\left(\mathfrak{X}_{\eta}\right)$, one has $\left.R^{q} \Theta(F)\right|_{\mathfrak{Y}_{s}} \stackrel{\sim}{\rightarrow} R^{q} \Theta\left(\left.F\right|_{\mathfrak{Y}_{\eta}}\right), q \geq 0$.

(ii) For a morphism $\varphi: \mathfrak{Y} \rightarrow \mathfrak{X}$ in $k^{\circ}-\mathcal{F}$ sch and $F^{\cdot} \in D^{+}\left(\mathfrak{Y}_{\eta}\right)$, one has

$$
R \Theta\left(R \varphi_{\eta_{*}} F\right) \stackrel{\sim}{\rightarrow} R \varphi_{s_{*}}(R \Theta(F)) .
$$

(iii) For $\mathfrak{X} \in k^{\circ}-\mathcal{F}$ sch and $F \in \mathbf{S}\left(\mathfrak{X}_{\eta}\right)$, there is a spectral sequence

$$
E_{2}^{p, q}=H^{p}\left(\mathfrak{X}_{s}, R^{q} \Theta(F)\right) \Longrightarrow H^{p+q}\left(\mathfrak{X}_{\eta}, F\right) .
$$

For $\mathfrak{X} \in k^{\circ}-\mathcal{F}$ sch, let $\mathfrak{X}_{\bar{s}}$ (resp. $\mathfrak{X}_{\bar{\eta}}$ ) denote the closed (resp. generic) fibre of the formal scheme $\overline{\mathfrak{X}}:=\mathfrak{X}_{\mathbb{\otimes}^{\circ}}\left(\widehat{k^{s}}\right)^{\circ}$ over $\left(\widehat{k}^{s}\right)^{\circ}$. One has $\mathfrak{X}_{\bar{s}}=\mathfrak{X}_{s} \otimes \widetilde{k^{s}}$ and $\mathfrak{X}_{\bar{\eta}}=\mathfrak{X}_{\eta} \widehat{\otimes} \widehat{k^{s}}$. The vanishing cycles functor $\Psi_{\eta}: \mathfrak{X}_{\eta_{\bar{e}}} \rightarrow \mathfrak{X}_{\bar{s} \text { ét }}$ is defined by $\Psi_{\eta}(F)=\Theta_{\widehat{k^{s}}}(\bar{F})$, where $\bar{F}$ is the pullback of $F$ on $\mathfrak{X}_{\bar{\eta}}$. It is clear that there is a canonical action of the Galois group $G_{\eta}:=G\left(k^{s} / k\right)$ on $\Psi_{\eta}(F)$ compatible with the action of $G_{s}:=G\left(\widetilde{k}^{s} / \widetilde{k}\right)$ on $\mathfrak{X}_{\bar{s}}$. We shall see that this action is continuous (see [SGA7], Exp. XIII, §1.1). For this (and for further use) we introduce the following notations. Let $K$ be a field over $k$ with a valuation that extends the valuation on $k$. We denote by $\mathfrak{X}_{s_{K}}$ and $\ddot{X}_{\eta_{K}}$ the closed and the generic fibres of the formal scheme $\mathfrak{X}_{K}:=\mathfrak{X}_{\boldsymbol{}_{k}} \circ \widehat{K}^{\circ}$ over $\widehat{K}^{\circ}$. (One has $\mathfrak{X}_{s_{K}}=\mathfrak{X}_{s} \otimes \widetilde{K}$ and $\mathfrak{X}_{\eta_{K}}=\mathfrak{X}_{\eta} \widehat{\otimes} \widehat{K}$.) For an étale sheaf $F$ on $\mathfrak{X}_{\eta}$, let $F_{K}$ denote the pullback of $F$ on $\mathfrak{X}_{\eta_{K}}$.

Lemma 4.3. For an étale sheaf $F$ on $\mathfrak{X}_{\eta}$, one has $\Psi_{\eta}(F)=\lim _{\longrightarrow} \bar{i}_{K}^{*}\left(\Theta_{K}\left(F_{K}\right)\right)$, where $K$ runs through finite extensions of $k$ in $k^{s}$ and $\bar{i}_{K}$ denotes the canonical morphism $\mathfrak{X}_{\bar{s}} \rightarrow \mathfrak{X}_{s_{K}}$. In particular, the action of $G_{\eta}$ on $\Psi_{\eta}(F)$ is continuous.

Proof. Let $\mathfrak{Y}_{s}$ be a quasicompact scheme étale over $\mathfrak{X}_{9}$. Then the $k$-analytic space $\mathfrak{Y}_{\eta}$ is compact and, by [Ber2], 5.3.4, one has $F\left(\mathfrak{Y}_{\eta} \widehat{\otimes} \widehat{k^{s}}\right)=\lim _{\longrightarrow} F\left(\mathfrak{Y}_{\eta} \widehat{\otimes} K\right)$, where $K$ runs through finite extensions of $k$ in $k^{s}$. This gives the required statement because any scheme étale over $\mathfrak{X}_{\bar{s}}$ has an étale covering by schemes of the form $\mathfrak{Y}_{\bar{s}}$, where $\mathfrak{Y}_{s}$ is affine and étale over $\mathfrak{X}_{s}$. 
Thus, $\Psi_{\eta}$ is actually a functor from $\mathfrak{X}_{\eta_{\mathfrak{e}}}$ to the category of étale sheaves of sets on $\mathfrak{X}_{\bar{s}}$ endowed with a continuous action of $G_{\eta}$ compatible with the action of $G_{s}$ on $\mathfrak{X}_{s}\left(G_{\eta}\right.$-sheaves). Let $\mathbf{S}_{G_{\eta}}\left(\mathfrak{X}_{\bar{x}}\right)$ denote the category of étale abelian $G_{\eta}$-sheaves on $\mathfrak{X}_{\bar{s}}$. Then $\Psi_{\eta}$ defines a left exact functor $\mathbf{S}\left(\mathfrak{X}_{\eta}\right) \rightarrow \mathbf{S}_{G_{\eta}}\left(\mathfrak{X}_{\bar{s}}\right)$. Therefore it defines right exact functors $R^{q} \Psi_{\eta}: \mathbf{S}\left(\mathfrak{X}_{\eta}\right) \rightarrow \mathbf{S}_{G_{\eta}}\left(\mathfrak{X}_{\bar{s}}\right)$ and an exact functor between the corresponding derived categories $R \Psi_{\eta}: D^{+}\left(\mathfrak{X}_{\eta}\right) \rightarrow D_{G_{\eta}}^{+}\left(\mathfrak{X}_{\bar{s}}\right)$.

Lemma 4.4. Let $F$ be an étale abelian sheaf on $\mathfrak{X}_{\eta}$. Then

(i) $R^{q} \Psi_{\eta}(F)=\lim \bar{i}_{K}^{*}\left(R^{q} \Theta_{K}\left(F_{K}\right)\right)$, where $K$ runs through finite extensions of $k$ in $k^{s}$;

(ii) if $F$ is soft, then the sheaf $\Psi_{\eta}(F)$ is flabby.

Proof . (i) follows from Lemma 4.3. (ii) follows from Lemma 3.2 and Proposition 4.1(iii).

Corollary 4.5. (i) For an étale morphism $\mathfrak{Y} \rightarrow \mathfrak{X}$ in $k^{\circ}-\mathcal{F}$ sch and $F \in \mathbf{S}\left(\mathfrak{X}_{\eta}\right)$, one has $\left.R^{q} \Psi_{\eta}(F)\right|_{\mathfrak{Y}_{s}} \stackrel{\sim}{\rightarrow} R^{q} \Psi_{\eta}\left(\left.F\right|_{\mathfrak{Y}_{\eta}}\right), q \geq 0$.

(ii) For a morphism $\varphi: \mathfrak{Y} \rightarrow \mathfrak{X}$ in $k^{\circ} \cdot \mathcal{F}$ sch and $F^{\cdot} \in D^{+}\left(\mathfrak{Y}_{\eta}\right)$, one has

$$
R \Psi_{\eta}\left(R \varphi_{\eta_{*}} F^{*}\right) \stackrel{\sim}{\rightarrow} R \varphi_{\bar{s}_{*}}\left(R \Psi_{\eta}\left(F^{*}\right)\right) .
$$

(iii) For $\mathfrak{X} \in k^{\circ}-\mathcal{F}$ sch and $F \in \mathbf{S}\left(\mathfrak{X}_{\eta}\right)$, there is a spectral sequence

$$
E_{2}^{p, q}=H^{p}\left(\mathfrak{X}_{\bar{s}}, R^{q} \Psi_{\eta}(F)\right) \Longrightarrow H^{p+q}\left(\mathfrak{X}_{\bar{\eta}}, F\right) .
$$

The fact we are going to establish allows one to use induction reasoning in the calculation of the vanishing cycles sheaves.

Let $\mathfrak{X} \in k^{\circ}-\mathcal{F} s c h$, and suppose that the canonical morphism $\mathfrak{X} \rightarrow \operatorname{Spf}\left(k^{\circ}\right)$ goes through a morphism $\mathfrak{X} \rightarrow \mathfrak{T}:=\operatorname{Spf}\left(k^{\circ}\{T\}\right)$. We remark that $\mathfrak{T}$ is the formal completion of the affine line over $k^{\circ}$, and $\mathfrak{T}_{\eta}$ is the one dimensional unit disc over $k$. Let $t$ be the maximal point of $\mathfrak{T}_{\eta}$ (it corresponds to the norm of the $k$-affinoid algebra $k\{T\})$. Then the image $s^{\prime}$ of $t$ under the reduction map $\pi: \mathfrak{T}_{\eta} \rightarrow \mathfrak{T}_{s}$ is the generic point of $\mathfrak{T}_{s}, \pi^{-1}\left(s^{\prime}\right)=\{t\}$ and $\widetilde{\mathcal{H}(t)}=k\left(s^{\prime}\right)=\widetilde{k}(T)$. We set $\mathfrak{X}^{\prime}=\mathfrak{X} \times{ }_{\mathfrak{T}} \operatorname{Spf}\left(\mathcal{H}(t)^{\circ}\right)$. This is a formal scheme in $\mathcal{H}(t)^{\circ}-\mathcal{F} s c h$. Let $\mathfrak{X}_{s^{\prime}}^{\prime}$ and $\mathfrak{X}_{\eta^{\prime}}^{\prime}$ denote the closed and the generic fibres of $\mathfrak{X}^{\prime}$, and let $\Theta^{\prime}$ and $\Psi_{\eta^{\prime}}$ denote the corresponding vanishing cycles functors. The canonical morphism of formal schemes $\lambda: \mathfrak{X}^{\prime} \rightarrow \mathfrak{X}$ induces morphisms $\lambda_{s}: \mathfrak{X}_{s^{\prime}}^{\prime} \stackrel{\sim}{\rightarrow}\left(\mathfrak{X}_{s}\right)_{s^{\prime}} \rightarrow \mathfrak{X}_{s}$ and $\lambda_{\eta}: \mathfrak{X}_{\eta^{\prime}}^{\prime} \stackrel{\sim}{\rightarrow}\left(\mathfrak{X}_{\eta}\right)_{t} \rightarrow \mathfrak{X}_{\eta}$, where $\left(\mathfrak{X}_{s}\right)_{s^{\prime}}$ (resp. $\left.\left(\mathfrak{X}_{\eta}\right)_{t}\right)$ is the fibre of the morphism $\mathfrak{X}_{s} \rightarrow \mathfrak{T}_{s}$ (resp. $\mathfrak{X}_{\eta} \rightarrow \mathfrak{T}_{\eta}$ ) at the point $s^{\prime}$ (resp.t). The pullback of an étale sheaf $F$ on $\mathfrak{X}_{\eta}$ with respect to $\lambda_{\eta}$ is denoted by $F^{\prime}$. Furthermore, we fix an embedding of fields $k^{s} \hookrightarrow \mathcal{H}(t)^{s}$. It induces a morphism $\lambda_{\bar{s}}: \mathfrak{X}_{\overline{s^{\prime}}}^{\prime} \stackrel{\sim}{\rightarrow}\left(\mathfrak{X}_{\bar{s}}\right)_{\bar{s}^{\prime}} \rightarrow \mathfrak{X}_{\bar{s}}$.

Proposition 4.6. (i) For any étale abelian sheaf $F$ on $\mathfrak{X}_{\eta}$ and any $q \geq 0$, there is a canonical isomorphism

$$
\lambda_{s}^{*}\left(R^{q} \Theta(F)\right) \stackrel{\sim}{\rightarrow} R^{q} \Theta^{\prime}\left(F^{\prime}\right) .
$$

(ii) For any étale abelian torsion sheaf $F$ on $\mathfrak{X}_{\eta}$ with torsion orders prime to $p=\operatorname{char}(\tilde{k})$ and any $q \geq 0$, there is a canonical isomorphism 


$$
\lambda_{\bar{s}}^{*}\left(R^{q} \Psi_{\eta}(F)\right) \stackrel{\sim}{\rightarrow} R^{q} \Psi_{\eta^{\prime}}\left(F^{\prime}\right)^{P},
$$

where $P=G\left(\mathcal{H}(t)^{s} / \mathcal{H}(t)^{\mathrm{n} r} k^{s}\right)$ (it is a pro-p-group).

Proof . (i) Since the morphism $\lambda_{\eta}: \mathfrak{X}_{\eta^{\prime}}^{\prime} \rightarrow \mathfrak{X}_{\eta}$ is a quasi-immersion, from Lemma 3.2 it follows that if $F$ is a soft sheaf, then the sheaf $\lambda_{\eta}^{*} F$ is also soft. Therefore it suffices to verify that $\lambda_{s}^{*}(\Theta(F)) \stackrel{\sim}{\rightarrow} \Theta^{\prime}\left(F^{\prime}\right)$. Furthermore, since the statement is local with respect to $\mathfrak{X}$, we may assume that $\mathfrak{X}$ is affine. We remark that any affine scheme étale over $\mathfrak{X}_{s^{\prime}}^{\prime}$ is of the form $\mathfrak{Y}_{s^{\prime}}^{\prime}$, where $\mathfrak{Y}$ is an affine formal scheme étale over $\mathfrak{X}$, and the sheaf $\lambda_{s}^{*}(\Theta(F))$ is associated with the presheaf $\lambda_{s}^{p}(\Theta(F))$ whose value at $\mathfrak{Y}_{s^{\prime}}^{\prime}$ as above coincides with the inductive limit $\lim \Theta(F)\left(\mathfrak{Y}_{s} \times \mathfrak{T}_{s} \mathfrak{U}_{s}\right)$, where $\mathfrak{U}_{s}$ runs through open neighborhoods of the generic point $s^{\prime}$ in $\mathfrak{T}_{s}$. We have $\lambda_{s}^{p}(\Theta(F))\left(\mathfrak{Y}_{s^{\prime}}^{\prime}\right)=$ $\underset{\longrightarrow}{\lim } F\left(\mathfrak{Y}_{\eta} \times{ }_{\mathfrak{T}_{\eta}} \mathfrak{U}_{\eta}\right)$. The $k$-analytic spaces $\mathfrak{Y}_{\eta} \times \mathfrak{T}_{\eta} \mathfrak{U}_{\eta}$ are identified with analytic domains in $\mathfrak{Y}_{\eta}$ and their intersection coincides with $\mathfrak{Y}_{\eta^{\prime}}^{\prime}=\left(\mathfrak{Y}_{\eta}\right)_{t}$. It follows that the set considered coincides with $F\left(\left(\mathfrak{Y}_{\eta}\right)_{t}\right)=\lambda_{\eta}^{*} F\left(\mathfrak{Y}_{\eta^{\prime}}^{\prime}\right)=\Theta^{\prime}\left(F^{\prime}\right)\left(\mathfrak{Y}_{s^{\prime}}^{\prime}\right)$.

(ii) Let $K$ be a Galois extension of $k$ in $k^{s}$ that contains $k^{\text {nr }}$, the maximal unramified extension $k$. The residue field $\widetilde{K}$ is a purely inseparable extension of $\widetilde{k}^{s}$, and there is a surjective continuous homomorphism $G(K / k) \rightarrow G_{s}$. Therefore one can define, for $\mathfrak{X} \in k^{\circ}-\mathcal{F} s c h$, a left exact functor $\Psi_{\eta, K}$ from $\mathfrak{X}_{\eta_{\hat{e}}}$ to the category of étale $G(K / k)$ sheaves on $\mathfrak{X}_{s_{K}}$. It induces a left exact functor $\Psi_{\eta, K}: \mathbf{S}\left(\mathfrak{X}_{\eta}\right) \rightarrow \mathbf{S}_{G(K / k)}\left(\mathfrak{X}_{s_{K}}\right)$ and the right derived functors $R^{q} \Psi_{\eta, K}: \mathbf{S}\left(\mathfrak{X}_{\eta}\right) \rightarrow \mathbf{S}_{G(K / k)}\left(\mathfrak{X}_{s_{K}}\right)$. (For example, from Corollary $4.5(i)$ it follows that $R^{q} \Psi_{\eta, k^{\operatorname{nr}}}(F)$ coincides with the pullback of $R^{a} \Theta(F)$ on $\mathfrak{X}_{s} \otimes \widetilde{k}^{s}$.) For a closed subgroup $I$ of $G_{\eta}$ which is contained in the inertia group of $k$, the values of the right derived functors of the functor $\mathbf{S}_{G_{\eta}}\left(\mathfrak{X}_{\bar{s}}\right) \rightarrow \mathbf{S}_{G_{\eta} / I}\left(\mathfrak{X}_{\bar{s}}\right): F \mapsto F^{I}$ are denoted by $\mathcal{H}(I, F)$.

Lemma 4.7. For $F \in \mathbf{S}\left(\mathfrak{X}_{\eta}\right)$ there is a spectral sequence

$$
E_{2}^{p, q}=\mathcal{H}\left(G\left(k^{*} / K\right), R^{q} \Psi_{\eta}(F)\right) \Longrightarrow \bar{i}_{K}^{*}\left(R^{p+q} \Psi_{\eta, K}(F)\right) .
$$

Proof . Let $\mathfrak{Y}_{s}$ be a quasicompact scheme étale over $\mathfrak{X}_{s}$. Then $F\left(\mathfrak{Y}_{\bar{\eta}}\right)^{G\left(k^{s} / K\right)}=$ $F\left(\mathfrak{Y}_{\eta_{K}}\right)$ and, if $F$ is injective, then $F\left(\mathfrak{Y}_{\bar{\eta}}\right)$ is a flabby $G_{\eta}$-module. The required fact follows.

Corollary 4.8. Let I be the inertia group of $k$. Then for $F \in \mathbf{S}\left(\mathfrak{X}_{\eta}\right)$ there is a spectral sequence $E_{2}^{p, q}=\mathcal{H}^{p}\left(I, R^{q} \Psi_{\eta}(F)\right) \Longrightarrow \bar{i}_{k}^{*}\left(R^{p+q} \Theta(F)\right)$.

First of all we verify that $P$ is a pro-p-group. For this it suffices to show that $\mathcal{H}(t)^{\mathrm{mr}}=\mathcal{H}(t)^{\mathrm{nr}} k^{\mathrm{mr}}$, where $k^{\mathrm{mr}}$ and $\mathcal{H}(t)^{\mathrm{mr}}$ are the maximal moderately ramified extensions of $k$ and $\mathcal{H}(t)$, respectively. By [Ber2], 2.4.4, the group $G\left(k^{\mathrm{mr}} / k^{\mathrm{nr}}\right)$ (resp. $\left.G\left(\mathcal{H}(t)^{\mathrm{mr}} / \mathcal{H}(t)^{\mathrm{nr}}\right)\right)$ is isomorphic to $\operatorname{Hom}\left(\sqrt{\left|k^{*}\right|} /\left|k^{*}\right|, \widetilde{k}^{{ }^{*}}\right)$ (resp. Hom $\left(\sqrt{\left|\mathcal{H}(t)^{*}\right|} /\right.$ $\left.\left|\mathcal{H}(t)^{*}\right|, \widehat{\mathcal{H}(t)^{s}}{ }^{*}\right)$ ). Since $\left|\mathcal{H}(t)^{*}\right|=\left|k^{*}\right|$, then $\sqrt{\left|\mathcal{H}(t)^{*}\right|}=\sqrt{\left|k^{*}\right|}$, and the required fact follows.

From (i) and Lemma 4.4(i) it follows that $\lambda_{s}^{*}\left(R^{q} \Psi_{\eta}(F)\right) \stackrel{\sim}{\rightarrow} \bar{i}_{K^{\prime}}^{\prime}\left(R^{q} \Psi_{\eta^{\prime}, K^{\prime}}\left(F^{\prime}\right)\right)$, where $K^{\prime}=\mathcal{H}(t)^{\mathrm{nr}} k^{. s}$ and $\bar{i}_{K^{\prime}}^{\prime}$ is the canonical morphism $\mathfrak{X}_{\bar{s}^{\prime}}^{\prime} \rightarrow \mathfrak{X}_{s^{\prime}{ }^{\prime}}^{\prime}$. The required isomorphism is obtained from Lemma 4.7 using the facts that $P$ is a pro-p-group and $F$ is a torsion sheaf with torsion orders prime to $p$. 
We will show now that the formation of vanishing cycles is compatible with extensions of the ground field.

Let $\mathfrak{X} \in k^{\circ}-\mathcal{F} s c h$. Let $K$ be a non-Archimedean field over $k$, and let $\Psi_{\eta_{K}}$ denote the vanishing cycles functor corresponding to the formal schemes $x_{K}$ over $K^{\circ}$. We fix an embedding of fields $k^{s} \hookrightarrow K^{s}$ and denote by $g$ the canonical morphism $\mathfrak{X}_{\bar{s}} \rightarrow \mathfrak{X}_{\bar{s}}$.

Theorem 4.9. Let $F$ be an étale abelian torsion sheaf on $\mathfrak{X}_{\eta_{1}}$ with torsion orders prime to $p=\operatorname{char}(\widetilde{k})$. Then for any $q \geq 0$ there is a canonical isomorphism

$$
g^{*}\left(R^{q} \Psi_{\eta}(F)\right) \stackrel{\sim}{\longrightarrow} R^{q} \Psi_{\eta_{K}}\left(F_{K}\right)
$$

Proof. We may assume that $\mathfrak{X}=\operatorname{Spf}(A)$, where $A$ is topologically finitely presented over $k^{\circ}$. The statement is proved by induction on $d=\operatorname{dim}\left(\dddot{X}_{s}\right)$. Suppose first that $d=0$. Since the homomorphism $\widetilde{A} \rightarrow \widetilde{\mathcal{A}}$, where $\mathcal{A}=A \otimes_{k} \circ k$, is finite (see $\S 1$ ), then $\operatorname{dim}(\widetilde{\mathcal{A}})=0$. Therefore $\operatorname{dim}\left(\mathfrak{X}_{\eta}\right)=0$, and the statement easily follows. Suppose now that $d \geq 1$ and that the statement is true for formal schemes whose closed fibres have dimension at most $d-1$. We remark that since the statement is true if $K$ is the completion of the algebraic closure of $k$, we may assume that the fields $k$ and $K$ are algebraically closed.

Step 1. The homomorphism $g^{*}\left(R^{q} \Psi_{\eta}(F)\right) \rightarrow R^{q} \Psi_{\eta_{K}}\left(F_{K}\right)$ induces an isomorphism at geometric points over any nonclosed point $x \in \mathfrak{X}_{s}$.

We can find a morphism $\mathfrak{X} \rightarrow \mathfrak{T}=\operatorname{Spf}\left(k^{\circ}\{T\}\right)$ such that the image $s^{\prime}$ of the point $x$ is the generic point of $\mathfrak{T}_{s}$. Let $\mathfrak{X}_{K} \rightarrow \mathfrak{T}_{K}=\operatorname{Spf}\left(K^{\circ}\{T\}\right)$ be the induced morphism, and let $s_{K}^{\prime}$ be the generic point of $\mathfrak{T}_{s_{K}}$. Furthermore, let $t$ and $t_{K}$ be the maximal points of $\mathfrak{T}_{\eta}$ and $\mathfrak{T}_{\eta_{K}}$, respectively. We set $\mathfrak{X}^{\prime}=\mathfrak{X} \times \mathfrak{T} \operatorname{Spf}\left(\mathcal{H}(t)^{\circ}\right)$ and $\mathfrak{X}_{K}^{\prime}=\mathfrak{X}_{K} \times \mathfrak{T}_{K} \operatorname{Spf}\left(\mathcal{H}\left(t_{K}\right)^{\circ}\right)$. Finally, let $\mathfrak{X}_{s^{\prime}}^{\prime}$ and $\mathfrak{X}_{\eta^{\prime}}^{\prime}$ (resp. $\mathfrak{X}_{s_{K}^{\prime}}^{\prime}$ and $\mathfrak{X}_{\eta_{K}^{\prime}}^{\prime}$ ) denote the closed and the generic fibres of $X^{\prime}$ (resp $\mathfrak{X}_{K}^{\prime}$ ). We fix a embedding of fields $\mathcal{H}(t)^{s} \hookrightarrow \mathcal{H}\left(t_{K}\right)^{s}$ over the canonical embedding $\mathcal{H}(t) \hookrightarrow \mathcal{H}\left(t_{K}\right)$. It induces a commutative diagram

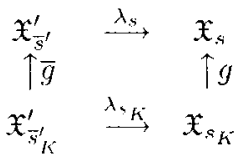

To prove the statement of Step 1 , it suffices to show that $\lambda_{s_{K}}^{*} q^{*}\left(R^{a} \Psi_{\eta}(F)\right) \stackrel{\sim}{\rightarrow}$ $\lambda_{s_{K}}^{*}\left(R^{q} \Psi_{\eta_{K}}\left(F_{K}\right)\right)$. By Proposition 4.6(ii), we have

$$
\lambda_{s_{K}}^{*} g^{*}\left(R^{q} \Psi_{\eta}(F)\right)=\bar{g}^{*} \lambda_{s}^{*}\left(R^{q} \Psi_{\eta}(F)\right) \stackrel{\sim}{\rightarrow} \bar{g}^{*}\left(R^{q} \Psi_{\eta^{\prime}}\left(F^{\prime}\right)\right)^{P^{\prime}},
$$

where $P=G\left(\mathcal{H}(t)^{s} / \mathcal{H}(t)^{\mathrm{nr}}\right)$, and

$$
\lambda_{s_{K}}^{*}\left(R^{q} \Psi_{\eta_{K}}\left(F_{K}\right)\right) \stackrel{\sim}{\rightarrow} R^{q} \Psi_{\eta_{K}^{\prime}}\left(F_{K}^{\prime}\right)^{Q}
$$

where $Q=G\left(\mathcal{H}\left(t_{K}\right)^{s} / \mathcal{H}\left(t_{K}\right)^{\mathrm{nr}}\right)$. On the other hand, by the induction hypothesis, we have

$$
\bar{g}^{*}\left(R^{q} \Psi_{\eta^{\prime}}\left(F^{\prime}\right)\right) \stackrel{\sim}{\rightarrow} R^{q} \Psi_{\eta_{K}^{\prime}}\left(F_{K}^{\prime}\right) .
$$

Therefore our statement follows from the following lemma. 
Lemma 4.10. The canonical homomorphism $Q \rightarrow P$ is surjective.

Proof. It suffices to show that for any finite extension $L$ of $\mathcal{H}(t)^{\mathrm{nr}}$ in $\mathcal{H}(t)^{s}$ one has $\left[L^{\prime}: \mathcal{H}\left(t_{K}\right)^{\mathrm{nr}}\right]=\left[L: \mathcal{H}(t)^{\mathrm{nr}}\right]$, where $L^{\prime}=L \mathcal{H}\left(t_{K}\right)^{\mathrm{nr}}$. We remark that the fields $\mathcal{H}(t)$ and $\mathcal{H}\left(t_{K}\right)$ are the completions of the fraction fields of $k\{T\}$ and $K\{T\}$, respectively. By Grauert-Remmert-Gruson Theorem ([BGR], 5.3.2/1), the latter fields are stable (see [BGR], §3.6). Therefore the fields $\mathcal{H}(t)$ and $\mathcal{H}\left(t_{K}\right)$ are stable. It follows that the fields $\mathcal{H}\left(t_{K}\right)^{\mathrm{nr}}$ and $\mathcal{H}\left(t_{K}\right)^{\mathrm{nr}}$ are also stable. Since $\widehat{\mathcal{H}(t)}=\widetilde{k}(T)$ and $\widehat{\mathcal{H}\left(t_{K}\right)}=\widetilde{K}(T)$, it follows that

$$
\left[L^{\prime}: \mathcal{H}\left(t_{K}\right)^{\mathrm{nr}}\right]=\left[\widetilde{L^{\prime}}: \widetilde{K}(T)^{s}\right] \text { and }\left[L: \mathcal{H}(t)^{\mathrm{nr}}\right]=\left[\widetilde{L}: \widetilde{k}(T)^{s}\right] .
$$

The field $\widetilde{L}^{\prime}$ contains the compositum $\widetilde{L} \widetilde{K}(T)^{s}$, and therefore

$$
\left[L^{\prime}: \mathcal{H}\left(t_{K}\right)^{\mathrm{nr}}\right] \leq\left[\widetilde{L} \widetilde{K}(T)^{s}: \widetilde{K}(T)^{s}\right] .
$$

Since the field $\widetilde{K}(T)^{s}$ is separable over $\widetilde{k}(T)^{s}$, the latter number is equal to $[\widetilde{L}$ : $\left.\widetilde{k}(T)^{s}\right]=\left[L: \mathcal{H}(t)^{\mathrm{nr}}\right]$. The required statement follows.

Step 2. $g^{*}\left(R^{q} \Psi_{\eta}(F)\right) \rightarrow R^{q} \Psi_{\eta_{K}}\left(F_{K}\right)$ is an isomorphism.

Let $\Delta$ be defined by the exact triangle in $D\left(\mathfrak{X}_{s_{K}}\right)$

$$
\longrightarrow g^{*}\left(R \Psi_{\eta}(F)\right) \longrightarrow R \Psi_{\eta_{K}}\left(F_{K}\right) \longrightarrow \Delta \longrightarrow
$$

We have to show that $\Delta$ is quasi-isomorphic to zero. By Step 1, the cohomology sheaves of $\Delta$ are concentrated at closed points of $\mathfrak{X}_{s_{K}}$. Therefore it suffices to show that $R \Gamma\left(\mathfrak{X}_{s_{K}}, \Delta\right)=0$, i.e., that $R \Gamma\left(\mathfrak{X}_{s_{K}}, g^{*}\left(R \Psi_{\eta}(F)\right)\right) \stackrel{\sim}{\rightarrow} R \Gamma\left(\mathfrak{X}_{s_{K}}, R \Psi_{\eta_{K}}\left(F_{K}\right)\right)$. By the invariance of étale cohomology of schemes under separably closed extensions of the ground field, the first complex is isomorphic to $R \Gamma\left(\mathfrak{X}_{s}, R \Psi_{\eta}(F)\right)$. By Corollary 4.5(ii), we have

$$
R \Gamma\left(\mathfrak{X}_{s}, R \Psi_{\eta}(F)\right)=R \Gamma\left(\mathfrak{X}_{\eta}, F\right) \text { and } R \Gamma\left(\mathfrak{X}_{s_{K}}, R \Psi_{\eta_{K}}\left(F_{K}\right)\right)=R \Gamma\left(\mathfrak{X}_{\eta_{K}}, F_{K}\right) .
$$

Therefore the required statement follows from the invariance of étale cohomology of analytic spaces under algebraically closed extensions of the ground field ([Ber2], 7.6.1).

\section{The comparison theorem for vanishing cycles}

First of all we recall the definition of the vanishing cycles functor for schemes (see [SGA7], Exp. XIII). Let $\mathcal{S}$ be the spectrum of a local Henselian ring which is the ring of integers $k^{\circ}$ of a field $k$ with a valuation. (Usually one considers the case when the valuation is discrete and nontrivial, but everything works in the more general situation.) The scheme $\mathcal{S}$ consists of the closed point $s=\operatorname{Spec}(\widetilde{k})$ and the generic point $\eta=\operatorname{Spec}(k)$. (If the valuation is trivial, then $s=\eta$.) Since the ring $k^{\circ}$ is Henselian, then the field $k$ is quasicomplete (see [Ber2], §2.4). This means that the valuation on $k$ extends uniquely to the separable closure $k^{s}$, and therefore the integral closure of $k^{\circ}$ in $k^{s}$ is also a local Henselian ring that coincides with $\left(k^{s}\right)^{\circ}$. We set $\overline{\mathcal{S}}=\operatorname{Spec}\left(\left(k^{s}\right)^{\circ}\right)=\{\bar{s}, \bar{\eta}\}$. For a scheme $\mathcal{X}$ over $\mathcal{S}$, let $\mathcal{X}_{s}$ and $\mathcal{X}_{\eta}$ (resp. $\mathcal{X}_{\bar{s}}$ and $\mathcal{X}_{\bar{\eta}}$ ) 
be the closed and the generic fibres of $\mathcal{X}\left(\right.$ resp. $\overline{\mathcal{X}}:=\mathcal{X} \times_{\mathcal{S}} \overline{\mathcal{S}}$ ). One has canonical morphisms

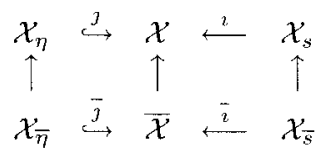

The vanishing cycles functor $\Psi_{\eta}: \mathcal{X}_{\eta_{\text {ét }}}^{\sim} \rightarrow \mathcal{X}_{\bar{s} \text { ét }}^{\sim}$ is defined by $\Psi_{\eta}(\mathcal{F})=\bar{i}^{*}\left(\bar{j}_{*} \overline{\mathcal{F}}\right)$, where $\overline{\mathcal{F}}$ is the pullback of a sheaf $\mathcal{F} \in \mathcal{X}_{\eta_{\text {ét }}}^{\sim}$ on $\mathcal{X}_{\bar{\eta}}$. The functor $\Psi_{\eta}$ takes values in the category of étale sheaves on $\mathcal{X}_{\bar{s}}$ that are endowed with a continuous action of $G_{\eta}:=G\left(k^{s} / k\right)$ compatible with the action of $G_{s}:=G\left(\widetilde{k}^{s} / \widetilde{k}\right)$ on $\mathcal{X}_{\bar{s}}$. (If the valuation on $k$ is trivial, then $\Psi_{\eta}(\mathcal{F})=\overline{\mathcal{F}}$.)

Furthermore, if the valuation on $k$ is nontrivial, we fix a non-zero element $a$ in the maximal ideal $k^{\circ \circ}$ of $k^{\circ}$. If the valuation on $k$ is trivial, we set $a=0$. The completion $\widehat{k}$ of $k$ with respect to the valuation is a non-Archimedean field, and one has $\widehat{k}^{\circ}:=(\widehat{k})^{\circ}=\lim k^{\circ} /\left(a^{n}\right)$.

Let now $\mathcal{X}$ be a scheme locally finitely presented over $k^{\circ}$. Then the formal completion $\hat{\mathcal{X}}$ of $\mathcal{X}$ along the subscheme $\left(\mathcal{X}, \mathcal{O}_{\mathcal{X}} / a \mathcal{O}_{\mathcal{X}}\right)$ is a formal scheme from $\hat{k}^{\circ}-\mathcal{F} s c h$, and one has the generic fibre $\widehat{\mathcal{X}}_{\eta}$. One the other hand, one can consider the $\widehat{k}$-analytic space $\mathcal{X}_{\eta}^{\text {an }}:=\left(\mathcal{X}_{\eta} \otimes_{k} \widehat{k}\right)^{\text {an }}$. From the construction of these two spaces it follows that there is a canonical morphism $\widehat{\mathcal{X}}_{\eta} \rightarrow \mathcal{X}_{\eta}^{\text {an }}$. We claim that if $\mathcal{X}$ is separated and finitely presented over $k^{\circ}$, then this morphism identifies $\widehat{\mathcal{X}}_{\eta}$ with a closed analytic domain in $\mathcal{X}_{\eta}^{\text {an }}$. Indeed, if $\mathcal{X}=\operatorname{Spec}(A)$, where $A$ is finitely presented over $k^{\circ}$, and $f_{1}, \ldots, f_{n}$ generate $A$ over $k^{\circ}$, then $\hat{\mathcal{X}}_{\eta}$ is identified with the affinoid domain $\left\{x \in \mathcal{X}_{\eta}^{\text {an }}|| f_{l}(x) \mid \leq 1,1 \leq i \leq n\right\}$. If $\mathcal{X}$ is arbitrary, we take a finite covering $\left\{\mathcal{X}_{\imath}\right\}_{\eta} \in I$ of $\mathcal{X}$ by open affine subschemes of the above form. Then for any pair $i, j \in I$ the intersection $\mathcal{X}_{\imath \jmath}=\mathcal{X}_{\imath} \cap \mathcal{X}_{j}$ is also an affine scheme and the canonical morphism $\mathcal{X}_{\imath \jmath} \rightarrow$ $\mathcal{X}_{2} \times \mathcal{X}_{j}$ is a closed immersion. It follows that if $f_{1}, \ldots, f_{n}$ and $g_{1}, \ldots, g_{m}$ generate the rings $\mathcal{O}\left(\mathcal{X}_{\imath}\right)$ and $\mathcal{O}\left(\mathcal{X}_{j}\right)$ over $k^{\circ}$, respectively, then $f_{1}, \ldots, f_{n}, g_{1}, \ldots, g_{m}$ generate $\mathcal{O}\left(\mathcal{X}_{\imath \jmath}\right)$ over $k^{\circ}$. It follows that $\widehat{\mathcal{X}}_{\imath, \eta}$ is identified with the intersection $\widehat{\mathcal{X}}_{\imath, \eta} \cap \widehat{\mathcal{X}}_{\jmath, \eta}$, and therefore $\widehat{\mathcal{X}}_{\eta}$ is identified with a closed analytic domain in $\mathcal{X}_{\eta}^{\text {an }}$. We claim that a proper morphism $\varphi: \mathcal{Y} \rightarrow \mathcal{X}$ induces an isomorphism $\widehat{\mathcal{Y}}_{\eta} \stackrel{\sim}{\rightarrow} \mathcal{Y}_{\eta}^{\text {an }} \times x_{\mathcal{X}_{\eta}} \widehat{\mathcal{X}}_{\eta}$. (In particular, the induced morphism $\widehat{\varphi}_{\eta}: \widehat{\mathcal{Y}}_{\eta} \rightarrow \widehat{\mathcal{X}}_{\eta}$ is proper, and if $\mathcal{X}$ is proper over $k^{\circ}$, then $\widehat{\mathcal{X}}_{\eta} \stackrel{\sim}{\rightarrow} \mathcal{X}_{\eta}^{\text {an }}$.) Indeed, since the statement is local with respect to $\mathcal{X}$, we may assume that $\mathcal{X}=\operatorname{Spec}(A)$, where $A$ is finitely presented over $k^{\circ}$. Then $\hat{\mathcal{Y}}_{\eta}$ and $\mathcal{Y}_{\eta}^{\text {an }} \times \mathcal{X}_{\eta}^{\text {an }} \widehat{\mathcal{X}}_{\eta}$ are closed analytic domains in $\mathcal{Y}_{\eta}^{\text {an }}$. Therefore it suffices to verify that the morphism considered is surjective. This is easily seen if $\mathcal{Y}$ is the projective space over $\mathcal{X}$ or a closed subscheme of $\mathcal{X}$ and, therefore, if $\varphi$ is a projective morphism. If $\varphi$ is arbitrary, this is obtained by applying Chow's Lemma that tells that there exist a projective $\mathcal{X}$-scheme $\mathcal{Z}$ and a projective surjective $\mathcal{X}$-morphism $\mathcal{Z} \rightarrow \mathcal{Y}$.

We remark that there are canonical morphisms of sites $\widehat{\mathcal{X}}_{\eta_{\text {ét }}} \rightarrow \mathcal{X}_{\eta}^{\text {an }}{ }_{\text {ét }} \rightarrow \mathcal{X}_{\eta_{\text {ét }}}$. The pullbacks of a sheaf $\mathcal{F} \in \mathcal{X}_{\eta_{\hat{e}}}$ on $\mathcal{X}_{\eta}^{\text {an }}$ and $\widehat{\mathcal{X}}_{\eta}$ will be denoted by $\mathcal{F}^{\text {an }}$ and $\widehat{\mathcal{F}}$, respectively. For any étale sheaf $\mathcal{F}$ on $\mathcal{X}_{\eta}$ there is a canonical morphism of sheaves on $\mathcal{X}_{s}, i^{*}\left(j_{*} \mathcal{F}\right) \rightarrow \Theta(\hat{\mathcal{F}})$. Indeed, the first sheaf is associated with the presheaf 
$i^{p}\left(j_{*} \mathcal{F}\right)$ that assigns to a scheme $\mathcal{Z}$ étale over $\mathcal{X}_{s}$ the inductive $\operatorname{limit} \lim \mathcal{F}\left(\mathcal{Y}_{\eta}\right)$ over all morphisms $\mathcal{Z} \rightarrow \mathcal{Y}_{s}$ over $\mathcal{X}_{s}$, where $\mathcal{Y}$ is a scheme étale over $\mathcal{X}$. If $\mathfrak{Z}$ is the formal scheme étale over $\hat{\mathcal{X}}$ with $\mathcal{Z}_{s}=\mathcal{Z}$, then such a morphism $\mathcal{Z} \rightarrow \mathcal{Y}_{s}$ induces a morphism of formal schemes $\mathfrak{Z} \rightarrow \widehat{\mathcal{Y}}$ over $\hat{\mathcal{X}}$, and therefore it induces a map $\mathcal{F}\left(\mathcal{Y}_{\eta}\right) \rightarrow \widehat{\mathcal{F}}\left(\boldsymbol{Z}_{\eta}\right)=\Theta(\widehat{\mathcal{F}})(\mathcal{Z})$. These maps define a morphism of presheaves $i^{p}\left(j_{*} \mathcal{F}\right) \rightarrow \Theta(\widehat{\mathcal{F}})$ that, in its turn, defines the required morphism of sheaves. It follows that there is a canonical morphism of sheaves $\Psi_{\eta}(\mathcal{F}) \rightarrow \Psi_{\eta \eta}(\widehat{\mathcal{F}})$.

Theorem 5.1. Let $\mathcal{F}$ be an étale abelian torsion sheaf on $\mathcal{X}_{\eta}$. Then for any $q \geq 0$ there is a canonical isomorphism

$$
i^{*}\left(R^{q} j_{*} \mathcal{F}\right) \stackrel{\sim}{\rightarrow} R^{q} \Theta(\widehat{\mathcal{F}}) .
$$

Proof. We prove the statement by induction on $d=\operatorname{dim}\left(\mathcal{X}_{\eta}\right)$. (Since the statement is local with respect to $\mathcal{X}$, we may assume that $\mathcal{X}$ is finitely presented and, in particular, that $d<\infty$.) Suppose that $d \geq 1$ and that the theorem is true for schemes whose generic fibre has dimension at most $d-1$.

Step 1. The homomorphism $i^{*}\left(R^{q} j_{*} \mathcal{F}\right) \rightarrow R^{q} \Theta(\widehat{\mathcal{F}})$ induces an isomorphism at geometric points over any nonclosed point $x \in \mathcal{X}_{s}$.

We may assume that $\mathcal{X}$ is a closed subscheme of the affine scheme $A_{\mathcal{S}}^{m}$ over $\mathcal{S}$. Then there exists a projection $\mathcal{X} \rightarrow \mathcal{T}:=A_{\mathcal{S}}^{\mathrm{l}}$ such that the image of the point $x$ in $\mathcal{T}_{s}$ is the generic point $s^{\prime}$ of $\mathcal{T}_{s}$. To prove the statement, it suffices to show that the homomorphism $i^{*}\left(R^{q} j_{*} \mathcal{F}\right) \rightarrow R^{q} \Theta(\widehat{\mathcal{F}})$ induces an isomorphism between the pullbacks of the sheaves on $\left(\mathcal{X}_{s}\right)_{s^{\prime}}$.

Let $\mathcal{S}^{\prime}=\left\{s^{\prime}, \eta^{\prime}\right\}$ be the spectrum of the Henselization $\mathcal{O}_{\mathcal{T}, s^{\prime}}^{h}$ of the local ring $\mathcal{O}_{\mathcal{T}, s^{\prime}}$, and we set $\mathcal{X}^{\prime}=\mathcal{X} \times_{\mathcal{T}} \mathcal{S}^{\prime}$. The canonical morphism $\lambda: \mathcal{X}^{\prime} \rightarrow \mathcal{X}$

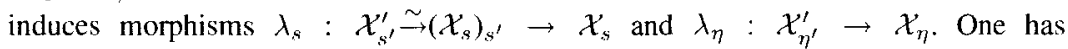
$\lambda_{s}^{*}\left(i^{*}\left(R^{q} j_{*} \mathcal{F}\right)\right) \stackrel{\sim}{\rightarrow} i^{\prime *}\left(R^{q} j_{*}^{\prime}\left(\lambda_{\eta}^{*} \mathcal{F}\right)\right)$. By induction, the latter sheaf is canonically isomorphic to $R^{q} \Theta^{\prime}\left(\widehat{\lambda_{\eta}^{*} \mathcal{F}}\right)$. Therefore there is a canonical isomorphism

$$
\lambda_{s}^{*}\left(i^{*}\left(R^{q} j_{*} \mathcal{F}\right)\right) \stackrel{\sim}{\rightarrow} R^{q} \Theta^{\prime}\left(\widehat{\lambda_{\eta}^{*} \mathcal{F}}\right) .
$$

On the other hand, let us consider the similar procedure for formal schemes. Let $t$ be the maximal point of $\widehat{\mathcal{T}}_{\eta}$. There is a canonical embedding of rings $\mathcal{O}_{T, s^{\prime}} \hookrightarrow \mathcal{H}(t)^{\circ}$, and it induces an isomorphism of the completion of $\mathcal{O}_{\mathcal{T}, s^{\prime}}$ with $\mathcal{H}(t)^{\circ}$. In particular, it gives rise to a canonical embedding of rings $\mathcal{O}_{\mathcal{T}, s^{\prime}}^{h} \hookrightarrow \mathcal{H}(t)^{\circ}$, and one has $\widehat{\mathcal{S}}^{\prime}=$ $\operatorname{Spf}\left(\mathcal{H}(t)^{\circ}\right)$. Furthermore, there is an isomorphism of formal schemes $\hat{\mathcal{X}}^{\prime} \stackrel{\sim}{\rightarrow} \widehat{\mathcal{X}} \times_{\widehat{\mathcal{S}}} \widehat{\mathcal{S}}^{\prime}$. If $\hat{\lambda}$ denotes the induced morphism $\hat{\mathcal{X}}^{\prime} \rightarrow \hat{\mathcal{X}}$, then, by Proposition 4.6(i), there is a canonical isomorphism

$$
\widehat{\lambda}_{s}^{*}\left(R^{q} \Theta(\hat{\mathcal{F}})\right) \stackrel{\sim}{\rightarrow} R^{q} \Theta^{\prime}\left(\hat{\lambda}_{\eta}^{*} \hat{\mathcal{F}}\right) .
$$

Since $\widehat{\lambda}_{s}=\lambda_{s}$ and $\widehat{\lambda}_{\eta}^{*} \widehat{\mathcal{F}}=\widehat{\lambda_{\eta}^{*} \mathcal{F}}$, the required statement follows.

Step 2. $i^{*}\left(R^{q} j_{*} \mathcal{F}\right) \rightarrow R^{q} \Theta(\hat{\mathcal{F}})$ is an isomorphism. 
Since the statement is local with respect to $\mathcal{X}$, we may assume that $\mathcal{X}$ is affine, and after that we may assume that $\mathcal{X}$ is projective over $\mathcal{S}$. Let $\Delta$ be defined by the exact triangle in $D\left(\mathcal{X}_{s}\right)$

$$
\longrightarrow i^{*}\left(R j_{*} \mathcal{F}\right) \longrightarrow R \Theta(\widehat{\mathcal{F}}) \longrightarrow \Delta \longrightarrow
$$

We have to show that $\Delta^{\prime}$ is quasi-isomorphic to zero. By Step 1, the cohomology sheaves of $\Delta$ are concentrated at closed points of $\mathcal{X}_{s}$. Therefore it suffices to show that $R \Gamma\left(\mathcal{X}_{s} \otimes-\widetilde{k}^{\prime}, \Delta^{\prime}\right)=0$ for any finite unramified extension $k^{\prime}$ of $k$. Replacing $k$ by $k^{\prime}$, we see that our problem is to show that $R \Gamma\left(\mathcal{X}_{s}, \Delta\right)=0$, i.e., that $R \Gamma\left(\mathcal{X}_{s}, i^{*}\left(R j_{*} \mathcal{F}\right)\right) \stackrel{\sim}{\rightarrow} R I\left(\mathcal{X}_{s}, R \Theta(\widehat{\mathcal{F}})\right)$.

Since $\mathcal{X}$ is proper over $\mathcal{S}$, there are canonical isomorphisms $R \Gamma\left(\mathcal{X}_{s}, i^{*}\left(R j_{*} \mathcal{F}\right)\right)$ $\stackrel{\sim}{\rightarrow} R \Gamma\left(\mathcal{X}_{\eta}, \mathcal{F}\right)$ and $R \Gamma\left(\mathcal{X}_{s}, R \Theta(\widehat{\mathcal{F}})\right) \stackrel{\sim}{\rightarrow} R \Gamma\left(\mathcal{X}_{\eta}^{\text {an }}, \mathcal{F}^{\text {an }}\right)$. Thus, the required fact follows from the following lemma.

Lemma 5.2. Let $\mathcal{X}$ be a compactifiable scheme over a quasicomplete field $k$, and let $\mathcal{F}$ be an abelian torsion sheaf on $\mathcal{X}$. Then for any $q \geq 0$ there is a canonical isomorphism

$$
H_{r}^{q}(\mathcal{X}, \mathcal{F}) \stackrel{\sim}{\rightarrow} H_{c}^{q}\left(\mathcal{X}^{\text {an }}, \mathcal{F}^{\text {an }}\right)
$$

Proof . By the Comparison Theorem 7.1.1 from [Ber2], the statement is true if $k$ is complete. Therefore it suffices to verify that $H_{c}^{q}(\mathcal{X}, \mathcal{F}) \stackrel{\sim}{\rightarrow} H_{c}^{q}(\mathcal{X} \otimes k, \widehat{k}, \mathcal{F})$. We know that this is true if $k$ is separably closed (in this case $\hat{k}$ is also separably closed). The general case is obtained using the Hochschield-Serre spectral sequence and the fact that the Galois groups of $k$ and $\widehat{k}$ are isomorphic ([Ber2], 2.4.2).

Corollary 5.3. For any étale abelian torsion sheaf $\mathcal{F}$ on $\mathcal{X}_{\eta}$ and any $q \geq 0$ there is a canonical isomorphism

$$
R^{q} \Psi_{\eta}(\mathcal{F}) \stackrel{\sim}{\rightarrow} R^{q} \Psi_{\eta}(\widehat{\mathcal{F}})
$$

Let $k$ be a non-Archimedean field. A morphism $\varphi: \mathfrak{Y} \rightarrow \mathfrak{X}$ in $k^{\circ}-\mathcal{F} s c h$ is said to be smooth if locally it goes through an étale morphism from $\mathfrak{Y}$ to the formal affine space $\mathbf{A}_{\mathfrak{X}}^{d}$ over $\mathfrak{X}$. A formal scheme $\mathfrak{X} \in k^{\circ}-\mathcal{F}$ sch is said to be smooth if the canonical morphism $\mathfrak{X} \rightarrow \operatorname{Spf}\left(k^{\circ}\right)$ is smooth.

Corollary 5.4. Let $\mathfrak{X}$ be a smooth formal scheme in $k^{\circ}-\mathcal{F}_{s c h}$, and let $n$ be an integer prime to $\operatorname{char}(\tilde{k})$. Then $\Psi_{\eta}(\mathbf{Z} / n \mathbf{Z})_{\mathfrak{X}_{\eta}}=(\mathbf{Z} / n \mathbf{Z})_{\mathfrak{X}_{\bar{s}}}$ and $R^{q} \Psi_{\eta}(\mathbf{Z} / n \mathbf{Z})_{\mathfrak{X}_{\eta}}=0$ for $q \geq 1$.

Proof . Corollary 4.5 (i) reduces a situation to the case when $\mathfrak{X}$ is the formal completion of the affine space $\mathbf{A}_{k^{\circ}}^{d}$. Therefore the statement follows from Corollary 5.3 and the known fact on the triviality of the vanishing cycles sheaves for smooth schemes.

Corollary 5.5. Suppose that $k$ is algebraically closed, and let $\mathcal{X}$ be a scheme of finite type over $k$ and $\mathcal{F}$ a constructible sheaf on $\mathcal{X}$ with torsion orders prime to $\operatorname{char}(\tilde{k})$. Then for any compact analytic domain $X$ in $\mathcal{X}^{\text {an }}$ the groups $H^{q}\left(X, \mathcal{F}^{\text {an }}\right), q \geq 0$, are finite. 
Proof. If $\left\{V_{2}\right\}_{2 \in I}$ is a finite covering of $X$ by closed analytic domains, then the spectral sequence of Corollary 3.6 implies that to prove the statement it suffices to show that all of the groups $H^{q}\left(V_{\imath_{1}} \cap \ldots \cap V_{\imath_{m}}, \mathcal{F}^{\text {an }}\right)$ are finite. Therefore we may assume that $\mathcal{X}=\operatorname{Spec}(A)$ is affine and $X$ is affinoid. Furthermore, since the cohomology groups are preserved under algebraically closed extensions of the ground field ([Ber2], 7.6.1), we can increase the field $k$ and assume that the valuation on $k$ is nontrivial and $X$ is strictly $k$-affinoid. Furthermore, let $f_{1}, \ldots, f_{n}$ be generators of $A$ over $k$ such that $X \subset V:=\left\{x \in \mathcal{X}^{\text {an }}|| f_{z}(x) \mid \leq 1,1 \leq i \leq n\right\}$. By Gerritzen-Grauert Theorem ([BGR], 7.3.5/1), $X$ is a finite union of rational domains in $V$. Therefore we may assume that $X$ is a rational domain in $V$, i.e., there exist elements $h, g_{1}, \ldots, g_{m} \in$ $A$ without common zeros in $V$ such that $X=\left\{x \in V|| g_{2}(x)|\leq| h(x) \mid, 1 \leq i \leq m\right\}$. Replacing $A$ by $A\left[\frac{1}{h}\right]$, we may assume that $X$ is a Weierstrass domain in $V$. Thus, we can find generators $f_{1}, \ldots, f_{n}$ of $A$ over $k$ such that $X=\left\{x \in \mathcal{X}^{\text {an }}|| f_{\imath}(x) \mid \leq\right.$ $1,1 \leq i \leq n\}$.

Let a be the kernel of the surjective homomorphism $k\left[T_{1}, \ldots, T_{n}\right] \rightarrow A: T_{l} \mapsto f_{l}$. It is an ideal of $k\left[T_{1}, \ldots, T_{n}\right]$ generated by polynomials $g_{1}, \ldots, g_{m}$. Multiplying all $g_{2}$ by a constant, we may assume that $g_{\imath} \in k^{\circ}\left[T_{1}, \ldots, T_{n}\right]$. Let $\mathbf{b}$ be the ideal of $k^{\circ}\left[T_{1}, \ldots, T_{n}\right]$ generated by $g_{1}, \ldots, g_{m}$, and we set $B=k^{\circ}\left[T_{1}, \ldots, T_{n}\right] / \mathbf{b}$. We get an affine scheme $\mathcal{Y}=\operatorname{Spec}(B)$ finitely presented over $k^{\circ}$ with $\mathcal{Y}_{\eta}=\mathcal{X}$ and $\widehat{\mathcal{Y}}_{\eta} \stackrel{\sim}{\rightarrow} X$. By Deligne's Theorem 3.2 from [SGA $4 \frac{1}{2}$ ], Th. finitude, the vanishing cycles sheaves $R^{q} \Psi_{\eta}(\mathcal{F})$ are constructible. (In the proof of Deligne's Theorem one assumed that $k^{\circ}$ is a discrete valuation ring, but the proof works for arbitrary $k^{\circ}$.) Furthermore, by $l o c$. cit., 1.10, the cohomology groups of $\mathcal{Y}_{s}$ with coefficients in a constructible sheaf are finite. Applying Corollary 5.3 and the spectral sequence 4.5 (iii), we get the required statement.

Corollary 5.6. Suppose that $k$ is algebraically closed, and let $X$ be a compact $k$ analytic space such that each point of $X$ has a neighborhood of the form $V_{1} \cup \ldots \cup V_{n}$, where each $V_{\imath}$ is a closed analytic domain admitting a quasi-étale morphism to the analytification of a scheme of finite type over $k$. Then for any finite locally constant sheaf $F$ on $X$ with torsion orders prime to $\operatorname{char}(\widetilde{k})$ the groups $H^{q}(X, F), q \geq 0$, are finite.

Proof . By the reasoning from the beginning of the proof of Corollary 5.5, the situation is reduced to the case when $X$ is $k$-affinoid and connected. Furthermore, we can find a finite étale Galois covering $\varphi: Y \rightarrow X$ such that the sheaf $\varphi^{*} F$ is constant. Applying the Hochschield-Serre spectral sequence for this covering, we see that the situation is reduced to the case when $F=(\mathbf{Z} / n \mathbf{Z})_{X}$ for some $n$ prime to char $(\tilde{k})$. Finally, shrinking $X$, we may assume that $X$ is an affinoid domain in a $k$-analytic space $Y$ for which there exists a separated étale morphism $\varphi: Y \rightarrow \mathcal{X}^{\text {an }}$, where $\mathcal{X}$ is an affine scheme of finite type over $k$.

Let $y \in X$ and let $\mathbf{x}$ be the image of the point $x=\varphi(y)$ in $\mathcal{X}$. The field $k(\mathbf{x})$ is everywhere dense in $\mathcal{H}(x)$, and $\mathcal{H}(y)$ is a finite separable extension of $\mathcal{H}(x)$. Therefore we can find a finite separable extension $K$ of $k(\mathbf{x})$ which embeds in $\mathcal{H}(y)$ and is everywhere dense in it. Take an arbitrary étale morphism of finite type between affine schemes $g: \mathcal{Y} \rightarrow \mathcal{X}$ for which there exists a point $\mathbf{y} \in \mathcal{Y}$ with $g(\mathbf{y})=\mathbf{x}$ and $k(\mathbf{y})=K$. The embedding of $K$ in $\mathcal{H}(y)$ defines a point $y^{\prime} \in \mathcal{Y}^{\text {an }}$. Since $K$ is everywhere dense in $\mathcal{H}(y)$, then $\mathcal{H}(y)=\mathcal{H}\left(y^{\prime}\right)$. By Theorem 3.4.1 from [Ber2], the $k$-germs $(Y, y)$ and 
$\left(\mathcal{Y}^{\mathrm{an}}, y^{\prime}\right)$ are isomorphic. Thus, we may assume that $X$ is an affinoid domain in $\mathcal{X}^{\text {an }}$, where $\mathcal{X}=\operatorname{Spec}(A)$ is an affine scheme of finite type over $k$. In this case the statement follows from Corollary 5.5 .

Remark 5.7. The natural conjecture is that, for any formal scheme $\mathfrak{X} \in k^{\circ}-\mathcal{F}_{\text {sch }}$ and any finite locally constant sheaf $F$ on $\mathfrak{X}_{\eta}$ with torsion orders prime to $\operatorname{char}(\tilde{k})$, the vanishing cycles sheaves $R^{q} \Psi_{\eta}(F)$ are constructible. To prove this, it would be enough to show that the statement of Corollary 5.6 is true for any compact $k$-analytic space $X$.

\section{A uniform topology on the set of morphisms of analytic spaces}

All the analytic spaces considered in this section are assumed to be Hausdorff.

Let $\mathbf{F}$ be a prime non-Archimedean field. (This means that the subfield generated by the image of $\mathbf{Z}$ is everywhere dense in $\mathbf{F}$, i.e., $\mathbf{F}$ is the field $\mathbf{Q}_{p}$ with the $p$-adic valuation or the field $\mathbf{Q}$ or $\mathbf{F}_{p}$ with the trivial valuation.) Recall that an analytic space over $\mathbf{F}$ is a pair $(k, X)$, where $k$ is a non-Archimedean field over $\mathbf{F}$ and $X$ is a $k$ analytic space, and a morphism $(K, Y) \rightarrow(k, X)$ is a pair consisting of an isometric embedding $k \hookrightarrow K$ and a morphism of $K$-analytic spaces $Y \rightarrow X \otimes_{k} K$ (see [Ber2], $\S 1.4)$. For brevity the pair $(k, X)$ is denoted by $X$ and is called an analytic space. Given an analytic function $f$ on an analytic space $X$, we set $\rho(f)=\max _{r \in X}|f(x)|$. For a morphism $\varphi: Y \rightarrow X$, we denote by $\varphi^{*}$ the induced homomorphism $\mathcal{O}(X) \rightarrow \mathcal{O}(Y)$. Furthermore, given analytic spaces $X$ and $Y$, we denote by $\operatorname{Mor}(Y, X)$ the set of morphisms $Y \rightarrow X$. If $X$ and $Y$ are over an analytic space $T$, then $\operatorname{Mor}_{T}(Y, X)$ denotes the subset of $T$-morphisms. Finally, let $\mathcal{G}(X)$ denote the group of automorphisms of $X$. (If $X$ is $k$-analytic, then such an automorphism induces an isometric automorphism of the field $k$.) If $X$ is over $T$, then $\mathcal{G}_{T}(X):=\mathcal{G}(X) \cap \operatorname{Mor}_{T}(X, X)$. Our purpose is to endow the set $\operatorname{Mor}(Y, X)$ with a uniform space structure.

Let $X$ be an analytic space. We introduce a set $\mathfrak{E}(X)$ as follows. An element $\varepsilon$ of $\mathfrak{E}(X)$ consists of a finite family $s(\varepsilon)=\left\{U_{\imath}\right\}_{\imath \in I}$ of compact analytic domains in $X$ and, for each $i \in I$, of finite sets of analytic functions $\left\{f_{\imath \jmath}\right\}_{\jmath \in J_{2}}$ on $U_{\imath}$ and of positive numbers $\left\{t_{\imath \jmath}\right\}_{\jmath \in J_{2}}$. Such an element $\varepsilon$ defines, for each analytic space $Y$, a relation on the set $\operatorname{Mor}(Y, X)$ as follows. Given two morphisms $\varphi, \psi: Y \rightarrow X$, we write $d(\varphi, \psi)<\varepsilon$ if $\varphi^{-1}\left(U_{\imath}\right)=\psi^{-1}\left(U_{\imath}\right)$ and $\rho\left(\varphi_{\imath}^{*} f_{\imath \jmath}-\psi_{\imath}^{*} f_{\imath \jmath}\right) \leq t_{\imath \jmath}$ for all $i \in I$ and $j \in J_{\imath}$, where $\varphi_{\imath}$ and $\psi_{2}$ are the induced morphisms $\varphi^{-1}\left(U_{\imath}\right) \rightarrow U_{\imath}$ (if $\varphi^{-1}\left(U_{2}\right)$ is empty, the above inequality is assumed to hold). The relations $d(\varphi, \psi)<\varepsilon$ define a uniform space structure and, in particular, a topology on $\operatorname{Mor}(Y, X)$. (If $Y$ is reduced, then $\operatorname{Mor}(Y, X)$ is Hausdorff.) We endow the group $\mathcal{G}(X)$ with the topology induced from $\operatorname{Mor}(X, X)$. It is easy to see that $\mathcal{G}(X)$ is really a topological group (not necessarily Hausdorff), and its topology is defined by the system of the subgroups $\mathcal{G}_{\mathbb{E}}(X)=\left\{\sigma \in \mathcal{G}(X) \mid \sigma\left(U_{\imath}\right)=U_{\imath}, \rho\left(\sigma_{\imath}^{*} f_{\imath \jmath}-f_{\imath \jmath}\right) \leq t_{\imath \jmath}\right\}$ for $\varepsilon \in \mathcal{E}(X)$ as above.

For example, if $K / k$ is an extension of non-Archimedean fields, then we get a topological group $\mathcal{G}_{k}(K)\left(=\mathcal{G}_{\mathcal{M}(k)}(\mathcal{M}(K))\right)$. If $K=\hat{k}^{a}$, it is canonically isomorphic to the Galois group $\mathrm{Gal}\left(k^{s} / k\right)$.

Furthermore, we introduce a partial ordering on $\mathfrak{E}(X)$ as follows. Given $\varepsilon, \delta \in$ $\mathfrak{E}(X)$, we write $\varepsilon \leq \delta$ if, for any pair of morphisms $\varphi, \psi: Y \rightarrow X$, the relation $d(\varphi, \psi)<\varepsilon$ implies $d(\varphi, \psi)<\delta$. For example, given $\varepsilon, \delta \in \mathfrak{E}(X)$, let inf $(\varepsilon, \delta)$ denote 
the element of $\mathfrak{E}(X)$ for which the families of analytic domains, of analytic functions and of positive numbers are unions of those for $\varepsilon$ and $\delta$. Then $\inf (\varepsilon, \delta) \leq \varepsilon, \delta$ and $\gamma \leq \inf (\varepsilon, \delta)$ for any $\gamma \in \mathfrak{E}(X)$ with $\gamma \leq \varepsilon, \delta$. We remark also that for each $\varepsilon \in \mathfrak{E}(X)$ there exists $\delta \leq \varepsilon$ such that $\delta$ is the infimum of elements defined by the triples $(U, f, t)$, where $U$ is an affinoid domain in $X, f \in \mathcal{O}(U)$ and $t>0$. Finally, for a finite family $\mathcal{U}=\left\{U_{\imath}\right\}_{\imath \in I}$ of compact analytic domains in $X$, we set $|\mathcal{U}|=\cup_{\imath} \in I U_{\imath}$.

Proposition 6.1. Let $\mathcal{U}=\left\{U_{\imath}\right\}_{t \in I}$ be a finite family of affinoid domains in $X$. Then for any $\varepsilon \in \mathfrak{E}(X)$ with $|s(\varepsilon)| \subset|\mathcal{U}|$ there exists $\delta \in \mathfrak{E}(X)$ with $\delta \leq \varepsilon$ and $s(\delta)=\mathcal{U}$.

Proof. The situation is easily reduced to the case when $X=\mathcal{M}(\mathcal{A})$ is affinoid and $\mathcal{U}=\{X\}$. We may also assume that $\varepsilon$ is defined by a triple $(U, h, t)$ with $\rho_{U}(h) \neq 0$. Suppose first that $U$ is a rational domain, i.e., $U=X\left(r_{\imath}^{-1} \frac{f_{z}}{g}\right)=\left\{x \in X|| f_{\imath}(x) \mid \leq\right.$ $\left.r_{2}|g(x)|\right\}$, where $f_{1}, \ldots, f_{n}, g$ are elements of $\mathcal{A}$ without common zeroes in $X$ and $r_{1}, \ldots, r_{n}>0$. It is easy to see that if a pair $\varphi, \psi: Y \rightarrow X$ satisfies the conditions $\rho\left(\varphi^{*} f_{\imath}-\psi^{*} f_{\imath}\right) \leq \alpha r_{\imath}, \rho\left(\varphi^{*} g-\psi^{*} g\right) \leq \alpha / 2$, where $\alpha=\min _{x \in U}|g(x)|$, then $\varphi^{-1}(U)=$ $\psi^{-1}(U)$. Furthermore, we can replace the element $h$ by a sufficiently close element of the form $h^{\prime} / g^{m}$, where $h^{\prime} \in \mathcal{A}$ and $m \geq 0$. If the pair $\varphi, \psi$ satisfies, in addition, the conditions $\rho\left(\varphi^{*} h^{\prime}-\psi^{*} h^{\prime}\right) \leq t \alpha^{m}$ and $\rho\left(\varphi^{*} g^{m}-\psi^{*} g^{m}\right) \leq t \alpha^{2 m} \rho_{U}\left(h^{\prime}\right)^{-1}$, then $\rho\left(\varphi^{*} h-\psi^{*} h\right) \leq t$, i.e., $d(\varphi, \psi)<\varepsilon$.

If $U$ is a finite union of rational domains, then the statement easily follows from the first case. Suppose now that $U$ is arbitrary, and let $X$ be $k$-affinoid. Then we can find a field of the form $K_{r}=\left\{\sum_{\nu} \lambda_{\nu} T^{\nu}\left|\lambda_{\nu} \in \mathbf{F},\right| \lambda_{\nu} \mid r^{\nu} \rightarrow 0\right.$ as $\left.|\nu| \rightarrow \infty\right\}$ such that $k_{r}:=k \widehat{\otimes}_{\mathbf{F}} K_{r}$ is a field and the spaces $X \widehat{\otimes} k_{r}=X \widehat{\otimes}_{\mathbf{F}} K_{r}$ and $U \widehat{\otimes} k_{r}$ are strictly $k_{r}$-affinoid (see [Berl], §2). There are canonical maps $\operatorname{Mor}(Y, X) \rightarrow$ $\operatorname{Mor}\left(Y \widehat{\otimes}_{\mathbf{F}} K_{r}, X \widehat{\otimes} k_{r}\right): \varphi \mapsto \varphi^{\prime}$ and $\mathfrak{E}(X) \mapsto \mathfrak{E}\left(X \widehat{\otimes} k_{r}\right): \varepsilon \mapsto \varepsilon^{\prime}$. We remark that if $d\left(\varphi^{\prime}, \psi^{\prime}\right)<\varepsilon^{\prime}$, then $d(\varphi, \psi)<\varepsilon$. By Gerritzen-Grauert Theorem ([BGR],7.3.5/3), $U \widehat{\otimes} k_{r}$ is a finite union of rational domains in $X \widehat{\otimes} k_{r}$. Applying the previous case, we can find an element $\gamma \in \mathfrak{E}\left(X \hat{\otimes} k_{r}\right)$ with $s(\gamma)=\left\{X \widehat{\otimes} k_{r}\right\}$ and $\gamma \leq \varepsilon^{\prime}$. Therefore the required statement follows from the following lemma.

Lemma 6.2. For any $\gamma \in \mathfrak{E}\left(X \widehat{\otimes} k_{r}\right)$ with $s(\gamma)=\left\{X \widehat{\otimes} k_{r}\right\}$ there exists $\delta \in \mathfrak{E}(X)$ with $s(\delta)=\{X\}$ such that, for any pair $\varphi, \psi \in \operatorname{Mor}(Y, X)$ with $d(\varphi, \psi)<\delta$, one has $d\left(\varphi^{\prime}, \psi^{\prime}\right)<\gamma$.

Proof. We may assume that $\gamma$ is defined by a function $g=\sum_{\nu} f_{\nu} T^{\nu} \in \mathcal{A} \widehat{\otimes} k_{r}$, $f_{\nu} \in \mathcal{A}$, and a positive number $t$. If $\varphi, \psi \in \operatorname{Mor}(Y, X)$, then $\rho\left(\varphi^{\prime *} g-\psi^{\prime *} g\right)=$ $\max _{\nu} \rho\left(\varphi^{*} f_{\nu}-\psi^{*} f_{\nu}\right) r^{\nu}$. Since $\left\|f_{\nu}\right\| r^{\nu} \rightarrow 0$ as $|\nu| \rightarrow \infty$, we can find $N$ such that, for any $\nu$ with $|\nu|>N$, one has $\rho\left(f_{\nu}\right) r^{\nu} \leq t$. It follows that if the pair $\varphi, \psi$ satisfies the conditions $\rho\left(\varphi^{*} f_{\nu}-\psi^{*} f_{\nu}\right) \leq t r^{-\nu}$ for $|\nu| \leq N$, then $\rho\left(\varphi^{\prime *} g-\psi^{\prime *} g\right) \leq t$.

Corollary 6.3. Suppose that $X=\mathcal{M}(\mathcal{A})$ is $k$-affinoid, and let $f_{1}, \ldots, f_{n}$ be a $k$ affinoid generating system of elements of $\mathcal{A}$. Then for any $\varepsilon \in \mathfrak{E}(X)$ there exist $f_{n+1}, \ldots, f_{m} \in k$ and $t_{1}, \ldots, t_{m}>0$ such that, for the element $\delta \in \mathfrak{E}(X)$ defined by $\left(X,\left\{f_{\imath}\right\},\left\{t_{\eta}\right\}\right)$, one has $\delta \leq \varepsilon$.

Proof. By Proposition 6.1, we may assume that $\varepsilon$ is defined by a triple of the form $(X, g, t), g \in \mathcal{A}$. Let $P \in k\left[T_{1}, \ldots, T_{n}\right]$ be a polynomial with $\rho\left(g-P\left(f_{1}, \ldots, f_{n}\right)\right) \leq$ 
$t$, and let $f_{n+1}, \ldots, f_{m}$ be the nonzero coefficients of $P$. It is clear that we can find $t_{1}, \ldots, t_{m}>0$ such that, for any pair $\varphi, \psi: Y \rightarrow X$ with $\rho\left(\varphi^{*} f_{2}-\psi^{*} f_{\imath}\right) \leq t_{2}$, $1 \leq i \leq m$, one has $\rho\left(\varphi^{*}\left(P\left(f_{1}, \ldots, f_{n}\right)\right)-\psi^{*}\left(P\left(f_{1}, \ldots, f_{n}\right)\right)\right) \leq t$. For such a pair $(\varphi, \psi)$, one has $\rho\left(\varphi^{*} g-\psi^{*} g\right) \leq t$.

Proposition 6.4. Let a $k$-analytic group $G$ act on a $k$-analytic space $X$, and let $\bar{X}=$ $X \widehat{\otimes} \widehat{k}^{a}$. Then the canonical homomorphisms $G(k) \rightarrow \mathcal{G}_{k}(X)$ and $G(k) \times \operatorname{Gal}\left(k^{s} / k\right) \rightarrow$ $\mathcal{G}_{k}(\bar{X})$ are continuous.

Lemma 6.5. A k-point $x$ of a $k$-analytic space $X$ is contained in the topological interior of any affinoid domain $U$ that contains $x$.

Proof. We may assume that $X$ is compact and there are affinoid domains $U_{1}, \ldots, U_{n} \in$ $X$ such that $x \in U_{1} \cap \ldots \cap U_{n}$ and $X=U_{\mathbf{1}} \cup \ldots \cup U_{n}$. For each $i$, one has $U \cap U_{2}=V_{1} \cup \ldots \cup V_{m}$ for some affinoid domains $V_{J} \subset U_{2}$. Since $x$ is contained in the topological interior in $U_{2}$ of each $V_{3}$ that contains $x$, then $x$ is contained in the topological interior of $U \cap U_{\imath}$ in $U_{\imath}$. It follows that $x$ is contained in the topological interior of $U$ in $X$.

Lemma 6.6. (i) If $X$ is $k$-analytic, then the space $\operatorname{Mor}_{k}(k, X)$ is homeomorphic to its image $X(k)$ in $X$.

(ii) Let $\varphi: Y \rightarrow X$ be a compact morphism. Then for any $\varepsilon \in \mathfrak{E}(X)$ there exists $\delta \in \mathfrak{E}(Y)$ such that, for any analytic space $Z$ and any pair of morphisms $\psi, \psi^{\prime}: Z \rightarrow$ $Y$ with $d\left(\psi, \psi^{\prime}\right)<\delta$, one has $d\left(\varphi \psi, \varphi \psi^{\prime}\right)<\varepsilon$.

(iii) Let $\psi: Z \rightarrow Y$ be a morphism. Then for any analytic space $X$, any $\varepsilon \in \mathfrak{E}(X)$ and any pair of morphisms $\varphi, \varphi^{\prime}: Y \rightarrow X$ with $d\left(\varphi, \varphi^{\prime}\right)<\varepsilon$, one has $d\left(\varphi \psi, \varphi^{\prime} \psi\right)<\varepsilon$.

(iv) Suppose that either $X$ and $Y$ are k-analytic and $Z$ is over $k$ or $X$ and $Y$ are over $k$ and $Z$ is $k$-analytic. Then the canonical map $\operatorname{Mor}_{k}(Y, X) \rightarrow \operatorname{Mor}_{k}\left(Y \times_{k}\right.$ $Z, X \times_{k} Z$ ) induces a uniform homeomorphism between the first space and its image in the second one.

Proof . (i) From Lemma 6.5 it follows that a basis of topology on $X(k)$ is formed by sets of the form $U(k)$ for affinoid domains $U \subset X$. Therefore the bijection $\operatorname{Mor}_{k}(k, X) \rightarrow X(k)$ is continuous. On the other hand, a basis of the topology on $X(k)$ induced from $\operatorname{Mor}_{k}(k, X)$ is formed by sets of the form $\{x \in U(k)|| f(x)-\alpha \mid \leq t\}$, where $f \in \mathcal{O}(O), \alpha \in k$ and $t>0$. The latter set is $V(k)$ for the affinoid domain $V=\{x \in U||(f-\alpha)(x) \mid \leq t\}$, and the required statement follows.

(ii) For $\varepsilon \in \mathfrak{E}(X)$ defined by a triple $(U, f, t)$, we define $\delta \in \mathfrak{E}(Y)$ by the triple $\left(\varphi^{-1}(U), \varphi^{*} f, t\right)$. It is easy to see that if $d\left(\psi, \psi^{\prime}\right)<\delta$, then $d\left(\varphi \psi, \varphi \psi^{\prime}\right)<\varepsilon$.

(iii) is trivial.

(iv) By Proposition 6.1, to show that the map is uniformly continuous, it suffices to verify that for any element $\varepsilon \in \mathfrak{E}\left(X \times_{k} Z\right)$ defined by a triple of the form $\left(U \times_{k} W, g, t\right)$, where $U \subset X$ and $W \subset Z$ are affinoid domains, there exists $\delta \in \mathfrak{E}(X)$ such that if $d(\varphi, \psi)<\delta$, then $d(\varphi \times$ id,$\psi \times$ id $)<\varepsilon$. We may assume that $g=\sum_{\imath=1}^{n} f_{2} \otimes h_{\imath}$ for $f_{\imath} \in \mathcal{O}(U)$ and $h_{\imath} \in \mathcal{O}(W), h_{\imath} \neq 0$. Then the necessary property is satisfied for $\delta$ defined by the affinoid domain $U$ and the systems of functions $\left\{f_{l}\right\}$ and of positive numbers $\left\{t /\left\|h_{\imath}\right\|\right\}$.

Furthermore, if $\delta \in \mathfrak{E}(X)$ is defined by a triple $(U, f, t)$, then we take an arbitrary compact analytic domain $W \subset Z$ and define $\varepsilon \in \mathfrak{E}\left(X \times_{k} Z\right)$ by the triple $\left(U \times_{k}\right.$ $W, f \otimes 1, t)$. It is easy to see that if $d(\varphi \times \mathrm{id}, \psi \times \mathrm{id})<\varepsilon$, then $d(\varphi, \psi)<\delta$. 
Proof of Proposition 6.4. Since $\mathcal{G}_{k}(X)$ and $\mathcal{G}_{k}(\bar{X})$ are homeomorphic to their images in $\operatorname{Mor}_{k}(X, X)$ and $\operatorname{Mor}_{k}(\bar{X}, \bar{X})$, respectively, and a basis of topology on $G(k)$ is formed by the sets $V(k)$, where $V$ is a compact analytic domain in $G$, then it suffices to verify that the maps $V(k) \rightarrow \operatorname{Mor}_{k}(X, X)$ and $V(k) \times \operatorname{Gal}\left(k^{s} / k\right) \rightarrow \operatorname{Mor}_{k}(\bar{X}, \bar{X})$ are continuous. The first map is the composition of continuous maps

$$
V(k)=\operatorname{Mor}_{k}(k, X) \rightarrow \operatorname{Mor}_{k}(X, V \times X) \rightarrow \operatorname{Mor}_{k}(X, X) .
$$

(The latter map is induced by the action morphism $G \times X \rightarrow X$, and the morphism $V \times X \rightarrow X$ is evidently compact.) From Lemma 6.6(iv) it follows now that the maps $V(k) \rightarrow \operatorname{Mor}_{k}(\bar{X}, \bar{X})$ and $\operatorname{Gal}\left(k^{s} / k\right) \rightarrow \operatorname{Mor}_{k}(\bar{X}, \bar{X})$ are continuous.

Corollary 6.7. Let $G$ be a $k$-analytic group. Then for any compact analytic domain $U \subset G$ the subgroup $G(k)_{U}:=\{g \in G(k) \mid g U=U\}$ is open in $G(k)$. Furthermore, the topology on $G(k)$ is defined by the system of subgroups $G(k)_{U}$, where $U$ runs through affinoid neighborhoods of the unity. Finally, the homomorphism $G(k) \rightarrow \mathcal{G}_{k}(G)$ defined by the left action of $G$ on itself induces a topological isomorphism of $G(k)$ with its image in $\mathcal{G}_{k}(G)$.

\section{The action of the set of morphisms on the étale cohomology groups}

As in $\S 6$, all the analytic spaces considered are assumed to be Hausdorff.

Theorem 7.1. Let $T$ be an analytic space, and let $F$ be a quasi-étale abelian sheaf on $T$. Then for any compact analytic space $X$ over $T$ and any element $\alpha \in H^{q}(X, F)$ there exists $\varepsilon \in \mathfrak{E}(X)$ such that, for any analytic space $Y$ over $T$ and any pair of $T$-morphisms $\varphi, \psi: Y \rightarrow X$ with $d(\varphi, \psi)<\varepsilon$, one has $\varphi^{*}(\alpha)=\psi^{*}(\alpha)$ in $H^{q}(Y, F)$.

Key Lemma 7.2. Let $X$ be an analytic space, and let $f: U \rightarrow X$ be a quasi-étale morphism with compact $U$. Then there exists $\varepsilon_{0} \in \mathcal{E}(U)$ with the following property. For any $\varepsilon \leq \varepsilon_{0}$ there exists $\delta \in \mathfrak{E}(X)$ such that, for any cartesian (resp. commutative) diagram

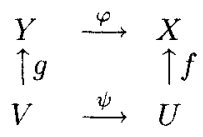

and any morphism $\varphi^{\prime}: Y \rightarrow X$ with $d\left(\varphi, \varphi^{\prime}\right)<\delta$, there exists a unique morphism $\psi^{\prime}: V \rightarrow U$ with $d\left(\psi, \psi^{\prime}\right)<\varepsilon$ for which the following diagram is also cartesian (resp. commutative)

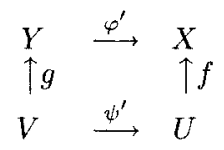

Proof. First of all we remark that the validity of the statement for commutative diagrams is easily deduced (using Lemma 6.6(iii)) from its validity for cartesian diagrams. And so we assume that the diagram $(*)$ is cartesian. 
Step 1. The statement is true if the spaces $X=\mathcal{M}(\mathcal{A})$ and $U=\mathcal{M}(\mathcal{B})$ are affinoid, the morphism $f$ is finite étale and $\mathcal{B}=\mathcal{A}[T] /(P)$, where $P=T^{n}+a_{1} T^{n-1}+\ldots+a_{n} \in$ $\mathcal{A}[T]$.

Lemma 7.3. (Generalized Krasner's Lemma) Let $f: U=\mathcal{M}(\mathcal{B}) \rightarrow \mathcal{M}(\mathcal{A})$ be a finite étale morphism of affinoid spaces such that $\mathcal{B}=\mathcal{A}[T] /(P)$, where $P=T^{n}+a_{1} T^{n-1}+$ $\ldots+a_{n} \in \mathcal{A}[T]$, and let $\alpha$ be the image of $T$ in $\mathcal{B}$. Then there exist positive numbers $r_{1}, \ldots, r_{n}, t$ and a series $\Phi \in \mathcal{B}\left\{r_{1}^{-1} S_{1}, \ldots, r_{n}^{-1} S_{n}\right\}$ with $\Phi(0)=\alpha$ such that, for any cartesian diagram $(*)$ with affinoid $Y=\mathcal{M}(\mathcal{C})$ and $V=\mathcal{M}(\mathcal{D})$ and for any polynomial $Q=T^{n}+c_{1} T^{n-1}+\ldots+c_{n} \in \mathcal{C}[T]$ with $\rho\left(c_{\imath}-\varphi^{*} a_{\imath}\right) \leq r_{\imath}, 1 \leq i \leq n$, the element $\beta=\left(\psi^{*} \Phi\right)\left(c_{1}-\varphi^{*} a_{1}, \ldots, c_{n}-\varphi^{*} a_{n}\right)$ is a unique root of $Q$ in $\mathcal{D}$ with $\rho\left(\beta-\psi^{*} \alpha\right)<t$ and the homomorphism $\mathcal{C}[T] \rightarrow \mathcal{D}: T \mapsto \beta$ induces an isomorphism $\mathcal{C}[T] /(Q) \stackrel{\sim}{\rightarrow} \mathcal{D}$.

Recall (see [Ray], Ch. XI) that if $I$ is an ideal in a commutative ring $A$, then the pair $(A, I)$ is called Henselian if it satisfies to any of the following equivalent conditions:

(a) $I \subset \operatorname{rad}(A)$ (the Jacobson radical of $A$ ) and for any monic polynomial $P \in$ $A[T]$ such that its image $\bar{P}$ in $\bar{A}[T](\bar{A}=A / I)$ is of the form $\overline{Q R}$, where $\bar{Q}$ and $\bar{R}$ are relatively prime monic polynomials in $\bar{A}[T]$, one has $P=Q R$ for some monic polynomials $Q, R \in A[T]$ whose images in $\bar{A}[T]$ are $\bar{Q}$ and $\bar{R}$, respectively;

(b) if $B$ is a finite free $A$-algebra, then $\operatorname{Idem}(B) \stackrel{\sim}{\rightarrow} \operatorname{Idem}(B / I B)$, where $\operatorname{Idem}(B)$ is the Boolean algebra of idempotent elements of $B$.

The following lemma is a generalization of Theorem 2.1.5 from [Ber2].

Lemma 7.4. Let $\Sigma$ be a closed subset of a paracompact analytic space $X$, and let $\mathcal{O}(\Sigma)$ be the algebra of functions analytic in a neighborhood of $\Sigma$. We set $I(\Sigma)=$ $\{f \in \mathcal{O}(\Sigma) \mid f(x)=0$ for all $x \in \Sigma\}$. Then the pair $(\mathcal{O}(\Sigma), I(\Sigma))$ is Henselian.

Proof . Let Idem $(\Sigma)$ denote the Boolean algebra of open-closed subsets of $\Sigma$. We claim that for any ideal $I \subset I(\Sigma)$ there are canonical isomorphisms

$$
\operatorname{Idem}(\mathcal{O}(\Sigma)) \stackrel{\sim}{\rightarrow} \operatorname{Idem}(\mathcal{O}(\Sigma) / I) \stackrel{\sim}{\rightarrow} \operatorname{Idem}(\Sigma)
$$

where the second map takes an idempotent $f \in \mathcal{O}(\Sigma) / I$ to the set of $x \in \Sigma$ with $f(x)=1$. Since a paracompact space is normal, it follows that the map from the first set to the third one is surjective. Therefore it suffices to verify that the both maps are injective. For this it suffices to show that $I(\Sigma) \subset \operatorname{rad}(\mathcal{O}(\Sigma)$ ). Suppose that an element $f \in I(\Sigma)$ is not contained in a maximal ideal $\mathbf{m} \subset \mathcal{O}(\Sigma)$. Then $1=f f^{\prime}+g g^{\prime}$ for some $g \in \mathbf{m}$ and $f^{\prime}, g^{\prime} \in \mathcal{O}(\Sigma)$. This implies that $g(x) \neq 0$ for all $x \in \Sigma$, i.e., $g$ is invertible in $\mathcal{O}(\Sigma)$.

To prove the lemma, we verify the condition (b). Let $B$ be a finite free $\mathcal{O}(\Sigma)$ algebra. Then we can shrink $X$ and assume that there is a finite free $\mathcal{O}(X)$-algebra $C$ such that $B=C \otimes \mathcal{O}(X) \mathcal{O}(\Sigma)$. The algebra $C$ gives rise to a finite morphism of analytic space $\varphi: Y \rightarrow X$ with $C=\mathcal{O}(Y)$. The space $Y$ is also paracompact, and one has $B=$ $\mathcal{O}\left(\pi^{-1}(\Sigma)\right)$. Since $I B \subset I\left(\pi^{-1}(\Sigma)\right)$, then $\operatorname{Idem}(B) \stackrel{\sim}{\rightarrow} \operatorname{Idem}(B / I B) \stackrel{\sim}{\rightarrow} \operatorname{Idem}\left(\pi^{-1}(\Sigma)\right)$, and therefore the condition (b) is satisfied.

Proof of Lemma 7.3. Of course, we may assume that $n>1$. Let $D\left(S_{1}, \ldots, S_{n}\right)=$ $\sum_{\nu} d_{\nu} S^{\nu}$ be the discriminant of the polynomial $\mathbf{P}=T^{n}+\left(a_{1}+S_{1}\right) T^{n-1}+\ldots+$ $\left(a_{n}+S_{n}\right) \in \mathcal{A}\left[S_{1}, \ldots, S_{n}\right][T]$. Since $d_{0}=D(0)$ is the discriminant of $P$, then $d_{0}$ is invertible in $\mathcal{A}$. Let $t$ be a positive number with 


$$
t^{2}|P|_{\mathrm{sp}}^{n^{2}-n-2}<\min _{x \in X}\left|d_{0}(x)\right|
$$

where $|P|_{\mathrm{sp}}=\max _{\imath} \rho\left(a_{\imath}\right)^{\frac{1}{2}}$ (the spectral norm of $P$ ). Then we can find $r_{1}, \ldots, r_{n}>0$ such that $\left\|d_{\nu}\right\| r^{\nu}<t$ for all $\nu \neq 0$ and $r_{z}<|P|_{\mathrm{sp}}^{2}$ for all $1 \leq i \leq n$.

Furthermore, let $Z \times E\left(0 ; r_{1}, \ldots, r_{n}\right) \subset \mathbf{A}^{n}$, where $E\left(0 ; r_{1}, \ldots, r_{n}\right)$ is the closed disc in $\mathbf{A}^{n}$, i.e., $Z=\mathcal{M}\left(\mathcal{B}\left\{r_{1}^{-1} S_{1}, \ldots, r_{n}^{-1} S_{n}\right\}\right)$, let $\Sigma=U \times\{0\} \subset Z$, and let $I$ be the ideal of $\mathcal{O}(\Sigma)$ generated by the coordinate functions $S_{1}, \ldots, S_{n}$. Then $I \subset I(\Sigma)$, and therefore the pair $(\mathcal{O}(\Sigma), I)$ is Henselian. We apply the condition (a) to the polynomial $\mathbf{P} \in \mathcal{O}(\Sigma)[T]$. One has $\mathcal{O}(\Sigma) / I=\mathcal{B}$ and $\overline{\mathbf{P}}=P=(T-\alpha) R$ for some monic polynomial $R \in \mathcal{B}[T]$. Since $\mathcal{B}=\mathcal{A}[T] /(P)$ is finite étale over $\mathcal{A}$, then the polynomials $T-\alpha$ and $R$ are relatively prime in $\mathcal{B}[T]$. Therefore $\mathbf{P}=(T-\Phi) \mathbf{R}$, where $\Phi \in \mathcal{O}(\Sigma)$ with $\Phi(0)=\alpha$ and $\mathbf{R}$ is a monic polynomial in $\mathcal{O}(\Sigma)[T]$ with $\overline{\mathbf{R}}=R$. We now can replace $r_{1}, \ldots, r_{n}$ by smaller positive numbers and assume that $\Phi \in \mathcal{B}\left\{r_{1}^{-1} S_{1}, \ldots, r_{n}^{-1} S_{n}\right\}, \mathbf{R} \in \mathcal{B}\left\{r_{1}^{-1} S_{1}, \ldots, r_{n}^{-1} S_{n}\right\}[T]$, and the norm of the element $\Phi-\alpha$ in $\mathcal{B}\left\{r_{1}^{-1} S_{1}, \ldots, r_{n}^{-1} S_{n}\right\}$ is strictly less than $t$. We claim that the required properties hold for the constructed $r_{1}, \ldots, r_{n}, t$ and $\Phi$.

Suppose we have a diagram (*) and a polynomial $Q$ as in the formulation. By the construction, the element $\beta$ is a root of $Q$ with $\rho\left(\beta-\psi^{*} \alpha\right)<t$ and the discriminant of $Q$ is invertible in $\mathcal{C}$. In particular, the homomorphism $\mathcal{C}[T] \rightarrow \mathcal{D}: T \mapsto \beta$ induces a finite étale homomorphism $\mathcal{C}[T] /(Q) \rightarrow \mathcal{D}$ over $\mathcal{C}$. Since the both algebras are free $\mathcal{C}$-modules of the same rank $n$, it follows that $\mathcal{C}[T] /(Q) \stackrel{\sim}{\rightarrow} \mathcal{D}$. It remains to show that if $\gamma$ is a root of $Q$ different from $\beta$, then $\rho\left(\gamma-\psi^{*} \alpha\right) \geq t$. For this we take an arbitrary bounded character $\chi: \mathcal{D} \rightarrow K$ to an algebraically closed non-Archimedean field $K$. The discriminant of the polynomial $\chi(Q)$ is equal to $\prod_{\imath<3}\left(\gamma_{l}-\gamma_{j}\right)^{2}$, where the product is taken over all pairs of roots of $\chi(Q)$ in $K$. Since $\left|\gamma_{\imath}\right| \leq|Q|_{\text {sp }} \leq|P|_{\text {sp }}$, our choice of $t$ guarantees that $\left|\gamma_{2}-\gamma_{j}\right|>t$ for all $i \neq j$. Since $\left|\chi\left(\beta-\psi^{*} \alpha\right)\right|<t$, it follows that $\left|\chi\left(\gamma-\psi^{*} \alpha\right)\right|>t$. Lemma 7.3 is proved.

We apply Lemma 7.3 to the morphism $f: U \rightarrow X$. Let $r_{1}, \ldots, r_{n}, t$ and $\Phi$ be given by the lemma, and let $\alpha$ be the image of $T$ in $\mathcal{B}$. Replacing $r_{\imath}$ by smaller numbers, we may assume that the norm of $\Phi-\alpha$ in $\mathcal{B}\left\{r_{1}^{-1} S_{1}, \ldots, r_{n}^{-1} S_{n}\right\}$ is less than $t$. We claim that the statement is true for $\varepsilon_{0}=(U, \alpha, t)$. Indeed, by Proposition 6.1 , it suffices to assume that $\varepsilon$ is the infimum of $\varepsilon_{0}$ and of an element $\varepsilon_{1}$ of the form $(U, h, q)$, where $h \in \mathcal{B}$ and $q>0$. The required element $\delta$ is defined as the infimum of the following families (1)-(3) of elements of $\mathfrak{E}(X)$.

(1) $\delta_{\imath}=\left(X, a_{\imath}, r_{\imath}\right), 1 \leq i \leq n$.

Before continuing we remark that the conditions $\delta \leq \delta_{\imath}, 1 \leq i \leq n$, are enough to construct, for any cartesian diagram $(*)$ and any morphism $\varphi^{\prime}: Y \rightarrow X$ with $d\left(\varphi, \varphi^{\prime}\right)<\delta$, a unique morphism $\psi^{\prime}: V \rightarrow U$ with $d\left(\psi, \psi^{\prime}\right)<\varepsilon_{0}$ for which the diagram $\left(*^{\prime}\right)$ is also cartesian. Indeed, to see this it suffices to assume that $Y=\mathcal{M}(\mathcal{C})$ and $V=\mathcal{M}(\mathcal{D})$ are affinoid. If $d\left(\varphi, \varphi^{\prime}\right)<\delta$, then $\rho\left(\varphi^{\prime *} a_{i}-\varphi^{*} a_{i}\right) \leq r_{\imath}, 1 \leq i \leq n$, and therefore the element $\beta^{\prime}:=\left(\psi^{*} \Phi\right)\left(\varphi^{\prime *} a_{1}-\varphi^{*} a_{1}, \ldots, \varphi^{\prime *} a_{n}-\varphi^{*} a_{n}\right)$ is a root of the polynomial $\varphi^{\prime *} P$. The homomorphism $\psi^{\prime *}: \mathcal{B} \rightarrow \mathcal{D}$ that extends $\varphi^{\prime *}: \mathcal{A} \rightarrow \mathcal{C}$ and takes $\alpha$ to $\beta^{\prime}$ is well defined and is a unique extension of $\varphi^{\prime *}$ with $\rho\left(\psi^{\prime *} \alpha-\psi^{*} \alpha\right)<t$. It is also clear that the diagram $\left(*^{\prime}\right)$ is cartesian.

Furthermore, let $h=b_{1} \alpha^{n-1}+\ldots+b_{n}$, where $b_{i} \in \mathcal{A}$.

(2) $\delta_{i}^{\prime}=\left(X, b_{i}, q \rho(\alpha)^{i-n}\right), 1 \leq i \leq n$. 
Finally, we can find $0<r_{\imath}^{\prime}<r_{z}, 1 \leq i \leq n$, such that the norm of the series $\Phi-\alpha$ in $\mathcal{B}\left\{r_{1}^{-1} S_{1}, \ldots, r_{n}^{\prime-1} S_{n}\right\}$ is at most $q \max \left\{\rho\left(b_{\imath}\right)^{-1} \rho(\alpha)^{1+\imath-n}\right\}$, where the maximum is taken over all $1 \leq i \leq n$ with $\rho\left(b_{i}\right) \neq 0$.

(3) $\delta_{2}^{\prime \prime}=\left(X, a_{\imath}, r_{\imath}^{\prime}\right), 1 \leq i \leq n$.

Let $\delta$ be the infimum of all the elements from (1)-(3). It suffices to verify that if $d(\varphi, \psi)<\delta$, then $d\left(\varphi^{\prime}, \psi^{\prime}\right)<\varepsilon_{1}$, i.e., $\rho\left(\psi^{\prime *} h-\psi^{*} h\right) \leq q$. One has

$$
\rho\left(\psi^{\prime *} h-\psi^{*} h\right) \leq \max _{1 \leq \imath \leq n}\left(\rho(\alpha)^{n-\imath} \rho\left(\varphi^{\prime *} b_{\imath}-\varphi^{*} b_{\imath}\right), \rho\left(b_{\imath}\right) \rho\left(\psi^{\prime *}(\alpha)^{n-\imath}-\psi^{*}(\alpha)^{n-\imath}\right)\right) .
$$

By (2), the first number under the maximum is at most $q$. Since $\rho\left(\psi^{\prime *}(\alpha)^{n-\imath}-\right.$ $\left.\psi^{*}(\alpha)^{n-\imath}\right) \leq \rho\left(\psi^{\prime *} \alpha-\psi^{*} \alpha\right) \rho(\alpha)^{n-\imath-1}$, then (3) implies that the second number is also at most $q$.

Step 2. The statement is true if $f$ identifies $U$ with an analytic domain in $X$.

In this case the statement is true even for arbitrary $\varepsilon \in \mathcal{E}(U)$. Indeed, let $\bar{\varepsilon}$ be the infimum of the two elements of $\mathfrak{E}(X)$ defined by the triple $(U, 0,1)$ and by the same families of affinoid domains, analytic functions and positive numbers as $\varepsilon$. It is easy to see that the necessary properties are satisfied for $\delta=\bar{\varepsilon}$.

Step 3. The statement is true in the general case.

Lemma 7.5. (i) If the statement is true for $U \rightarrow X$ and $U^{\prime} \rightarrow U$, then it is also true for the composition $U^{\prime} \rightarrow X$.

(ii) Let $\left\{X_{2}\right\}_{2 \in I}$ and $\left\{U_{2}\right\}_{2 \in I}$ be finite systems of closed analytic domains in $X$ and $U$ with $U=\cup_{\imath \in I} U_{\imath}$ and $f\left(U_{\imath}\right) \subset X_{\imath}$. If the statement is true for all the induced morphisms $f_{\imath}: U_{\imath} \rightarrow X_{\imath}$, then it is also true for $f$.

Proof . (i) is trivial.

(ii) By (i) and Step 2, we may assume that $X$ is compact and $X_{1}=X$ for all $i \in I$. It follows also that the statement is true for all the induced morphisms $f_{2 \jmath}: U_{2} \cap U_{\jmath} \rightarrow X$. Let $\varepsilon_{2,0}$ and $\varepsilon_{2 \jmath, 0}$ be the elements of $\mathfrak{E}\left(U_{2}\right)$ and $\mathfrak{E}\left(U_{1} \cap U_{\jmath}\right)$ for the morphisms $f_{2}$ and $f_{\imath \jmath}$. We claim that the statement is true for $\varepsilon_{0}:=\inf \left\{\bar{\varepsilon}_{2,0}, \bar{\varepsilon}_{\imath, 0}\right\}$, where $\bar{\varepsilon}_{\imath, 0}$ is the extension of $\varepsilon_{\imath, 0}$ to $U$ (as in Step 2). Indeed, let $\varepsilon \leq \varepsilon_{0}$, and let $\delta_{2}$ and $\delta_{23}$ be the elements of $\mathfrak{E}(X)$ which correspond to the pairs $\left(f_{2},\left.\varepsilon\right|_{U_{2}}\right)$ and $\left(f_{\imath \jmath},\left.\varepsilon\right|_{U_{2} \cap U_{j}}\right.$ ), where $\left.\varepsilon\right|_{U_{2}}$ is the restriction of $\varepsilon$ to $U_{2}$ (defined in the evident way). Suppose we are given a cartesian diagram (*) and a morphism $\psi: Y \rightarrow X$ with $d(\varphi, \varphi)<\delta:=\inf \left\{\delta_{i}, \delta_{2 j}\right\}$. Then $(*)$ induces diagrams $\left(*_{2}\right)$ and $\left(*_{1 j}\right)$ with $\left(f_{i}, \psi_{2}, V_{2}\right)$ and $\left(f_{\imath \jmath}, \psi_{\imath \jmath}, V_{\imath} \cap V_{j}\right)$, respectively, instead of $(f, \psi, V)$. By the assumptions, there exist unique morphisms $\psi_{2}^{\prime}: V_{\imath} \rightarrow U_{\imath}$ and $\psi_{\imath \jmath}^{\prime}: V_{\imath} \cap V_{\jmath} \rightarrow U_{\imath} \cap U_{\jmath}$ with $d\left(\psi_{2}, \psi_{1}^{\prime}\right)<\left.\varepsilon\right|_{\nu_{2}}$ and $d\left(\psi_{\imath \jmath}, \psi_{\imath \jmath}^{\prime}\right)<\left.\varepsilon\right|_{U_{2} \cap U_{3}}$ for which the corresponding diagrams $\left(*_{\imath}^{\prime}\right)$ and $\left(*_{\imath_{j}}^{\prime}\right)$ are cartesian. It follows that $\psi_{\imath \jmath}^{\prime}$ coincide with the restrictions of $\psi_{2}$ and $\psi_{2}^{\prime}$ to $V_{2} \cap V_{l}$, and therefore the morphisms $\psi_{2}^{\prime}$ are glued together to a morphism $\psi^{\prime}: V \rightarrow U$ for which $d\left(\psi, \psi^{\prime}\right)<\varepsilon$ and the diagram $\left(*^{\prime}\right)$ is cartesian.

We can find finite systems of affinoid domains $\left\{X_{\imath}\right\}_{\imath \in I}$ in $X$ and $\left\{U_{\imath}\right\}_{\imath \in I}$ in $U$ such that $U=\cup_{\imath \in I} U_{2}$ and, for each $i \in I, U_{2}$ is identified with an affinoid domain in an affinoid space finite and étale over $X_{2}$. Applying Lemma 7.5, we reduce the situation to the case when $f: U=\mathcal{M}(\mathcal{B}) \rightarrow X=\mathcal{M}(\mathcal{A})$ is a finite étale morphism of affinoid spaces. Then $f$ induces a finite étale morphism of affine $\operatorname{schemes} \operatorname{Spec}(\mathcal{B}) \rightarrow \operatorname{Spec}(\mathcal{A})$. 
From the local description of étale morphisms of schemes it follows that we can find, for each point $u \in U$, affinoid neighborhoods $U^{\prime}$ of $u$ and $X^{\prime}$ of $f(u)$ such that $f$ induces a finite étale morphism $U^{\prime} \rightarrow X^{\prime}$ for which $\mathcal{B}_{U^{\prime}}=\mathcal{A}_{X^{\prime}}[T] /(P)$, where $P$ is a monic polynomial in $\mathcal{A}_{X^{\prime}}[T]$. Applying Step 1 and Lemma 7.5, we get the required fact.

Proof of Theorem 7.1. First of all we verify the statement for $q=0$. Let $\alpha \in F(X)$. Then there is a finite quasi-étale covering $\left\{U_{n} \stackrel{f_{2}}{\rightarrow} X\right\}_{\imath \in I}$ with compact $U_{2}$ and, for each $i$, a commutative diagram

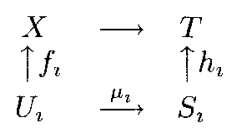

with quasi-étale $h_{\imath}$ and compact $S_{\imath}$ such that $f_{\imath}^{*}(\alpha)=\mu_{\imath}^{*}\left(\beta_{\imath}\right)$ for some $\beta_{\imath} \in F\left(S_{\imath}\right)$. We apply Key Lemma 7.2 to the morphisms $f_{2}: U_{\imath} \rightarrow X$ (for cartesian diagrams) and $h_{2}: S_{2} \rightarrow T$ (for commutative diagram). Let $\varepsilon_{2} \in \mathfrak{E}\left(U_{2}\right)$ and $\varepsilon_{1}^{\prime} \in \mathfrak{E}\left(S_{2}\right)$ be given by the theorem. Furthermore, applying Lemma 6.6(ii) to the compact morphism $\mu_{\imath}: U_{\imath} \rightarrow S_{\imath}$ and the element $\varepsilon_{\imath}^{\prime} \in \mathfrak{E}\left(S_{\imath}\right)$, we get an element $\delta_{\imath} \in \mathfrak{E}\left(U_{\imath}\right)$. By Key Lemma 7.2, we can find $\varepsilon \in \mathfrak{E}(X)$ such that for any system of cartesian diagrams

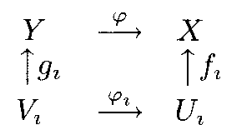

and any morphism $\psi: Y \rightarrow X$ with $d(\varphi, \psi)<\varepsilon$ there exists a unique system of morphisms $\psi_{\imath}: V_{\imath} \rightarrow U_{\imath}$ with $d\left(\varphi_{\imath}, \psi_{\imath}\right)<\inf \left\{\varepsilon_{\imath}, \delta_{\imath}\right\}$ for which the diagrams

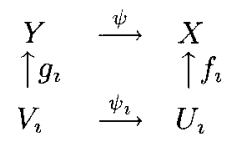

are also cartesian. We claim that $\varphi^{*}(\alpha)=\psi^{*}(\alpha)$ in $F(Y)$. Indeed, since $\left\{V_{\imath} \stackrel{g_{\imath}}{\rightarrow} Y\right\}_{\imath \in I}$ is a quasi-étale covering, it suffices to verify that $g_{2}^{*}\left(\varphi^{*}(\alpha)\right)=g_{2}^{*}\left(\psi^{*}(\alpha)\right)$ for all $i \in I$. One has $g_{\imath}^{*}\left(\varphi^{*}(\alpha)\right)=\varphi_{\imath}^{*}\left(f_{\imath}^{*}(\alpha)\right)=\varphi_{\imath}^{*}\left(\mu_{\imath}^{*}\left(\beta_{\imath}\right)\right)=\nu_{\imath}^{*}\left(\beta_{2}\right)$, where $\nu_{\imath}=\mu_{\imath} \varphi_{2}$ : $V_{i} \rightarrow S_{\imath}$. Similarly, one has $g_{i}^{*}\left(\psi^{*}(\alpha)\right)=\nu_{i}^{\prime *}\left(\beta_{\imath}\right)$, where $\nu_{\imath}^{\prime}=\mu_{\imath} \psi_{\imath}: V_{\imath} \rightarrow S_{\imath}$. Therefore it suffices to verify that $\nu_{2}=\nu_{\imath}^{\prime}$. Since $d\left(\varphi_{l}, \psi_{\imath}\right)<\delta_{2}$ then, by Lemma 6.6(ii), $d\left(\nu_{\imath}, \nu_{i}^{\prime}\right)<\varepsilon_{\imath}^{\prime}$. Key Lemma 7.2 applied to the morphism $h_{\imath}: T_{\imath} \rightarrow S_{\imath}$ and the canonical morphism $Y \rightarrow T$ implies that $\nu_{\imath}=\nu_{\imath}^{\prime}$.

To verify the statement for $q \geq 1$, we use the modified Čech procedure for calculation of the cohomology groups from [SGA4], Exp. V, §7. By loc. cit., 7.4.1, the group $H^{q}(X, F)$ is isomorphic to the inductive limit of the cohomology groups $H^{q}(K, F)$ over the category $\underline{\operatorname{HR}}_{q+1}$ of hypercoverings of $X$ of type $q+1$ up to homotopy.

Lemma 7.6. The family of the hypercoverings $K$., such that all the components $K_{n}$, $n \geq 0$, are representable by compact analytic spaces quasi-étale over $X$, is cofinal in $\underline{\mathrm{HR}}_{q+1}$.

Proof . Let $L$. be a hypercovering of $X$ from $\underline{H R}_{q+1}$. We construct $K$. that refines $L$. inductively. First of all, since $L_{0} \rightarrow X$ is a quasi-étale covering and $X$ is compact, 
we can find a quasi-étale covering $K_{0} \rightarrow X$ that refines $L_{0}$ and such that $K_{0}$ is representable by an affinoid space quasi-étale over $X$. Suppose now that, for some $0 \leq n \leq q$, we have already constructed a morphism $K^{(n)} \rightarrow i_{n}^{*} L$, where $K^{(n)}$ is an object of $\Delta^{\circ}[n] X_{\text {qét }}$ (we use the notations from loc.cit:; $X_{\text {qêt }}$ is the category of presheaves of sets on $X_{\text {qét }}$ ) such that all $K_{p}^{(n)}, 0 \leq p \leq n$, are representable by affinoid spaces quasi-étale over $X$ and the canonical morphisms $K_{p+1}^{(n)} \rightarrow\left(i_{p_{*}} K^{(n)}\right)_{p+1}$, $0 \leq p \leq n-1$, and $K_{0}^{(n)} \rightarrow X$ are quasi-étale coverings. The morphism $K^{(n)} \rightarrow i_{n}^{*} L$. induces a morphism $\left(i_{n *} K^{(n)}\right)_{n+1} \rightarrow\left(\operatorname{cosk}_{n}(L .)\right)_{n+1}$. Consider the cartesian diagram in $X_{\mathrm{q} \hat{\mathrm{c}} \mathrm{t}}$

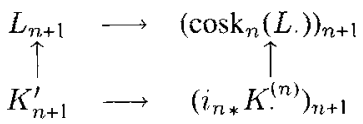

We remark that the category of compact analytic spaces quasi-étale over $X$ admits finite projective limits. In particular, $\left(i_{n *} K^{(n)}\right)_{n+1}$ is representable by a compact analytic space quasi-étale over $X$. Furthermore, since the upper arrow is a quasi-étale covering, the lower arrow is a quasi-étale covering too. Therefore, we can find a commutative diagram in $X_{\text {qềt }}$

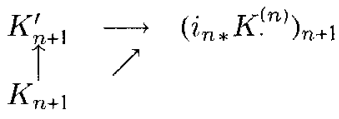

where $K_{n+1}$ is representable by an affinoid space quasi-étale over $X$ and $K_{n+1} \rightarrow$ $\left(i_{n *} K^{(n)}\right)_{n+1}$ is a quasi-étale covering. We define an object $K^{(n+1)} \in \Delta^{\circ}[n+1] X_{\text {qêt }}$ by $K_{p}^{(n+1)}=K_{p}^{(n)}$ for $0 \leq p \leq n$ and $K_{n+1}^{(n+1)}=K_{n+1}$. In this way we get a morphism $K^{(q+1)} \rightarrow i_{q+1}^{*} L$, where $K^{(q+1)} \in \Delta^{\circ}[q+1] X_{\text {qêt }}$ is such that all $K_{p}^{(q+1)}, 0 \leq p \leq q+1$, are representable by affinoid spaces quasi-étale over $X$ and the canonical morphisms $K_{p+1}^{(q+1)} \rightarrow\left(i_{p_{*}} K^{(q+1)}\right)_{p+1}, 0 \leq p \leq q$, and $K_{0}^{(q+1)} \rightarrow X$ are quasi-étale coverings. This morphism induces a morphism $K::=i_{q+1_{*}} K^{(q+1)} \rightarrow \operatorname{cosk}_{q+1}(L.) \stackrel{\sim}{\rightarrow} L$. It remains to note that all the components $K_{n}$ of $K$. are representable by compact analytic spaces quasi-étale over $X$.

Let $K$. be a hypercovering as in Lemma 7.6 such that $\alpha$ comes from the group $H^{q}(K, F)$, i.e., $\alpha$ comes from an element $\beta \in F\left(K_{q}\right)$. By Key Lemma 7.2 and the case $q=0$, we can find $\varepsilon \in \mathcal{E}(X)$ such that for any pair of $T$-morphisms $\varphi, \psi: Y \rightarrow X$ with $d(\varphi, \psi)<\varepsilon$ there is a canonical isomorphism of hypercoverings of $Y, \theta .: L^{(\varphi)}:=K, \times_{X, \varphi} Y \breve{\rightarrow} L_{.}^{(\not \psi)}:=K . \times_{X, \psi} Y$ with $\varphi_{q}^{*}(\beta)=\theta_{q}^{*}\left(\psi_{q}^{*}(\beta)\right)$, where $\varphi_{q}: L_{q}^{(\varphi)} \rightarrow K_{q}$ and $\psi_{q}: L_{q}^{(\psi)} \rightarrow K_{q}$. It follows that $\varphi^{*}(\alpha)=\psi^{*}(\alpha)$ in $H^{q}(Y, F)$. The theorem is proved.

Corollary 7.7. Let $T$ be an analytic space, and let $F$ be an étale abelian sheaf on $T$. Then for any $k$-analytic space $X$ over $T$ and any element $\alpha \in H_{c}^{q}(X, F)$ there exists $\varepsilon \in \mathfrak{E}(X)$ such that, for any pair of proper $T$-morphisms of $k$-analytic spaces $\varphi, \psi: Y \rightarrow X$ with $d(\varphi, \psi)<\varepsilon$, one has $\varphi^{*}(\alpha)=\psi^{*}(\alpha)$ in $H_{c}^{q}(Y, F)$.

Proof . By [Ber2], 5.2.8, one has

$$
H_{c}^{q}(X, F)=\lim _{r} H_{r}^{q}(\mathcal{U}, F),
$$


where $U$ runs through compact analytic domains in $X$ and $\mathcal{U}$ is the topological interior of $U$ in $X$. Suppose that $\alpha$ comes from an element $\beta \in H_{c}^{q}(\mathcal{U}, F)$ for some $U$. We remark that, for any proper morphism $\varphi: Y \rightarrow X, \varphi^{-1}(\mathcal{U})$ is the topological interior of $\varphi^{-1}(U)$ in $Y$ ([Ber2], 1.5.5). Let $\varepsilon_{1} \in \mathfrak{E}(X)$ be defined by the triple $(U, 0,1)$. Then for any pair of proper morphisms $\varphi, \psi: Y \rightarrow X$ with $d(\varphi, \psi)<\varepsilon_{1}$ one has $\varphi^{-1}(U)=\psi^{-1}(U)$ and, by the above remark, $\varphi^{-1}(\mathcal{U})=\psi^{-1}(\mathcal{U})$. Furthermore, by [Ber2], 5.2.5, one has $H_{c}^{q}(\mathcal{U}, F) \stackrel{\sim}{\rightarrow} H^{q}\left(U, j !\left(\left.F\right|_{\mathcal{U}}\right)\right.$ ), where $j$ is the open embedding $\mathcal{U} \hookrightarrow U$. Let $K$. be a hypercovering of $U$ as in Lemma 7.6 such that $\beta$ comes from the group $H^{q}\left(K, j_{!}\left(\left.F\right|_{\mathcal{U}}\right)\right)$, i.e., $\beta$ comes from an element $\gamma \in\left(j !\left(\left.F\right|_{\mathcal{U}}\right)\right)\left(K_{q}\right) \subset F\left(K_{q}\right)$. By Key Lemma 7.2 and the case $q=0$ of Theorem 7.1, we can find $\varepsilon \leq \varepsilon_{1}$ such that for any pair of proper $T$-morphisms $\varphi, \psi: Y \rightarrow X$ with $d(\varphi, \psi)<\varepsilon$ there is a canonical isomorphism $\theta .: L^{(\varphi)}:=K, \times_{X, \varphi} Y \stackrel{\sim}{\rightarrow} L^{(\psi)}:=K . \times_{X, \psi} Y$ of hypercoverings of $V:=\varphi^{-1}(U)=\psi^{-1}(U)$ with $\varphi_{q}^{*}(\gamma)=\theta_{q}^{*}\left(\psi_{q}^{*}(\gamma)\right)$ in $F\left(L_{q}^{(\varphi)}\right)\left(\varphi_{q}\right.$ and $\psi_{q}$ are the morphisms $L_{q}^{(\varphi)} \rightarrow K_{q}$ and $L_{q}^{(\psi)} \rightarrow K_{q}$, respectively). Since $\mathcal{V}:=\varphi^{-1}(\mathcal{U})=\psi^{*}(\mathcal{U})$, then the latter equality is in fact an equality in the subgroup $\left(j_{!}^{\prime}\left(\left.F\right|_{\mathcal{U}}\right)\right)\left(L_{q}^{(\varphi)}\right)$, where $j^{\prime}$ is the open embedding $\mathcal{V} \hookrightarrow V$. It follows that $\varphi^{*}(\beta)=\psi^{*}(\beta)$ in $H_{c}^{q}(\mathcal{V}, F)$, and therefore $\varphi^{*}(\alpha)=\psi^{*}(\alpha)$ in $H_{c}^{q}(Y, F)$.

Corollary 7.8. Let a k-analytic group $G$ act on a $k$-analytic space $X$, and let $A$ be a discrete Gal $\left(k^{s} / k\right)$-module. Then the cohomology groups $H_{c}^{q}(X, \Lambda)\left(\right.$ resp. $H^{q}(\bar{X}, A)$ ) are discrete $G(k)$ (resp. $\left.G(k) \times \mathrm{Gal}\left(k^{s} / k\right)\right)$ modules.

Remark 7.9. (i) The fact that $H_{c}^{q}(\bar{X}, F)$ are discrete $\mathrm{Gal}\left(k^{s} / k\right)$-modules for arbitrary étale abelian sheaves $F$ on $X$ follows from [Ber2], 5.3.5.

(ii) Given an analytic space $X$ and an abelian quasi-étale sheaf $F$ on $X$, one can endow the cohomology groups $H^{q}(X, F)$ with the topology with respect to which a basis of open subgroups is formed by the kernels of the homomorphisms $H^{q}(X, F) \rightarrow$ $H^{q}(U, F)$, where $U$ runs through compact analytic domains in $X$. From Theorem 7.1 it follows that in the situation of Corollary 7.8 the group $G(k)\left(\right.$ resp. $\left.G(k) \times \operatorname{Gal}\left(k^{s} / k\right)\right)$ acts continuously on the cohomology groups $H^{q}(X, \Lambda)$ (resp. $H^{q}(\bar{X}, A)$ ).

(iii) Using the same reasoning as in the proof of Theorem 7.1 and Corollary 7.7, one can prove the following fact. Let $F$ be an étale abelian sheaf on an analytic space $T$, and let $X$ and $Y$ be analytic spaces over $T$. Then for any element $\alpha \in H^{q}(Y, F)$ and any finite flat $T$-morphism $\varphi: Y \rightarrow X$ there exists $\varepsilon \in \mathfrak{E}(X)$ such that, for any finite flat $T$-morphism $\psi: Y \rightarrow X$ with $d(\varphi, \psi)<\varepsilon$, one has $\operatorname{Tr}_{\varphi}(\alpha)=\operatorname{Tr}_{\psi}(\alpha)$ in $H^{q}(X, F)$.

\section{The action of the set of morphisms of formal schemes on vanishing cycles}

Let $k$ be a quasicomplete field with nontrivial valuation, and let $a$ be a fixed nonzero element of the maximal ideal $k^{\circ \circ}$ of the ring of integers $k^{\circ}$. All the schemes considered below are assumed to be finitely presented over $k^{\circ}$. We fix such a scheme $\mathcal{T}$ (for example, $\mathcal{T}=\operatorname{Spec}\left(k^{\circ}\right)$ ), and let $\mathcal{F}$ be an étale abelian torsion sheaf on $\mathcal{T}_{\eta}$. From Comparison Theorem 5.1 it follows that if $\mathcal{X}$ and $\mathcal{Y}$ are schemes over $\mathcal{T}$, then any morphism of formal schemes $\varphi: \hat{\mathcal{Y}} \rightarrow \widehat{\mathcal{X}}$ over $\hat{\mathcal{T}}$ induces homomorphisms of sheaves on $\mathcal{Y}_{s}$ and $\mathcal{Y}_{\bar{s}}$, respectively, 


$$
\begin{gathered}
\theta^{q}(\varphi, \mathcal{F}): \varphi_{s}^{*}\left(i^{*}\left(R^{q} j_{*}\left(\left.\mathcal{F}\right|_{\mathcal{X}_{\eta}}\right)\right)\right)-i^{*}\left(R^{q} j_{*}\left(\left.\mathcal{F}\right|_{\mathcal{Y}_{\eta}}\right)\right) \\
\theta_{\eta}^{q}(\varphi, \mathcal{F}): \varphi_{\bar{s}}^{*}\left(R^{q} \Psi_{\eta}\left(\left.\mathcal{F}\right|_{\mathcal{X}_{\eta}}\right)\right) \longrightarrow R^{q} \Psi_{\eta}\left(\left.\mathcal{F}\right|_{\mathcal{Y}_{\eta}}\right)
\end{gathered}
$$

For a prime integer $l$, we set $s_{l}(k)=\operatorname{dim}_{\mathbf{F}_{l}}\left(\left|k^{*}\right| /\left|k^{*}\right|\right)$.

Theorem 8.1. Let $\mathcal{F}$ be a constructible sheaf on $\mathcal{T}_{\eta}$ with torsion orders prime to char $(\tilde{k})$, and suppose that $s_{l}(k)<\infty$ for each prime l dividing a torsion order of $\mathcal{F}$. Given $\hat{\mathcal{X}}$, where $\mathcal{X}$ is a scheme over $\mathcal{T}$, there exists $n \geq 1$ such that, for any scheme $\mathcal{Y}$ over $\mathcal{T}$ and any pair of $\widehat{\mathcal{T}}$-morphisms $\varphi, \psi: \widehat{\mathcal{Y}} \rightarrow \widehat{\mathcal{X}}$ that coincide modulo $a^{n}$, one has $\theta^{q}(\varphi, \mathcal{F})=\theta^{q}(\psi, \mathcal{F})$ for all $q \geq 0$.

Proof. First of all we verify that the sheaves considered are constructible.

Lemma 8.2. Let $l$ be a prime integer with $l \neq \operatorname{char}(\tilde{k})$ and $s_{l}(k)<\infty$. Then for any constructible l-torsion sheaf $\mathcal{G}$ on $\mathcal{X}_{\eta}$ the sheaves $i^{*}\left(R^{q} j_{*} \mathcal{G}\right)$ are constructible and equal to zero for $q>s_{l}(k)+2 \operatorname{dim}\left(\mathcal{X}_{\eta}\right)$.

Proof. By Deligne's Theorem 3.2 from [SGA $4 \frac{1}{2}$ ], Th. finitude, the vanishing cycles sheaves $R^{q} \Psi_{\eta}(\mathcal{G})$ are constructible. (In the proof of Deligne's Theorem one assumed that $k^{\circ}$ is a discrete valuation ring, but the proof works for arbitrary $k^{\circ}$.) It is clear that $R^{q} \Psi_{\eta}(\mathcal{G})=0$ for $q>2 \operatorname{dim}\left(\mathcal{X}_{\eta}\right)$. Let $I$ be the inertia group of $k$. Then there is a spectral sequence $\mathcal{H}^{p}\left(I, R^{q} \Psi_{\eta}(\mathcal{G})\right) \Longrightarrow \bar{i}^{*}\left(R^{p+q} j_{*} \mathcal{G}\right)$, where $\bar{i}: \mathcal{X}_{\bar{s}} \rightarrow \mathcal{X}_{s}$, and therefore it suffices to show that the sheaves $\mathcal{H}^{p}\left(I, R^{q} \Psi_{\eta}(\mathcal{G})\right)$ are constructible and are equal to zero for $p>s:=s_{l}(k)$. Let $Q$ be the minimal closed invariant subgroup of $I$ such that $M:=I / Q$ is a pro- $l$-group. Then the indices of all open subgroups of $Q$ are prime to $l$ and $M \stackrel{\sim}{\rightarrow} \mathbf{Z}_{l}^{s}$ (see [Ber2], 2.4.4). It follows that $\mathcal{H}^{p}\left(I, R^{q} \Psi_{\eta}(\mathcal{G})\right)=\mathcal{H}^{p}\left(M, R^{q} \Psi_{\eta}(\mathcal{G})^{Q}\right)$. Thus, our statement is reduced to the verification of the following simple fact. Let $\mathcal{G}$ be a constructible sheaf (on a scheme) endowed with a continuous action of the group $M \stackrel{\sim}{\rightarrow} \mathbf{Z}_{l}^{s}$. Then all the groups $\mathcal{H}^{p}(M, \mathcal{G})$ are constructible and are equal to zero for $p>s$. By induction, it suffices to consider the case $s=1$. Let $\sigma$ be a generator of $\mathbf{Z}_{l}$. Then $\mathcal{H}^{0}\left(\mathbf{Z}_{l}, \mathcal{G}\right)=\operatorname{Ker}(\mathcal{G} \stackrel{\sigma-1}{\longrightarrow} \mathcal{G}), \mathcal{H}^{1}\left(\mathbf{Z}_{l}, \mathcal{G}\right)=\operatorname{Coker}(\mathcal{G} \stackrel{\sigma-1}{\longrightarrow} \mathcal{G})$ and $\mathcal{H}^{p}\left(\mathbf{Z}_{l}, \mathcal{G}\right)=0$ for $p>1$.

We fix a functor $\mathfrak{U}_{s} \mapsto \mathfrak{U}$ from the category of schemes étale over $\mathcal{X}_{s}$ to the category of formal schemes étale over $\widehat{X}$ which is inverse to the functor from Lemma 2.1.

Corollary 8.3. In the situation of Lemma 8.2 suppose that the residue field $\widetilde{k}$ is separably closed. Then for any étale morphism of finite type $\mathfrak{U}_{s} \rightarrow \mathcal{X}_{s}$ the groups $H^{q}\left(\mathfrak{U}_{\eta}, \widehat{\mathcal{G}}\right)$ are finite.

Proof . By Comparison Theorem 5.1 and Corollary 4.2, there is a spectral sequence $E_{2}^{p, q}=H^{p}\left(\mathfrak{U}_{s}, i^{*}\left(R^{q} j_{*} \mathcal{G}\right)\right) \Longrightarrow H^{p+q}\left(\mathfrak{U}_{\eta}, \widehat{\mathcal{G}}\right)$. By Lemma 8.2 and [SGA4 $\left.\frac{1}{2}\right]$, Th. finitude, 1.10 , the groups $E_{2}^{p, q}$ are finite. The required statement follows.

Lemma 8.4. Let $\mathfrak{X}$ be a formal scheme finitely presented over $\hat{k}^{\circ}$. Then for any $\varepsilon \in \mathcal{E}\left(\mathfrak{X}_{\eta}\right)$ there exists $n \geq 1$ such that, for any formal scheme $\mathfrak{Y}$ locally finitely 
presented over $\widehat{k}^{\circ}$ and any pair of morphisms $\varphi, \psi: \mathfrak{Y} \rightarrow \mathfrak{X}$ that coincide modulo $a^{n}$, one has $d\left(\varphi_{\eta}, \psi_{\eta}\right)<\varepsilon$.

Proof . Suppose first that $\mathfrak{X}=\operatorname{Spf}(A)$, where $A$ is topologically finitely presented over $\widehat{k}^{\circ}$, and let elements $f_{1}, \ldots, f_{m}$ be the images of the coordinate functions under some surjective homomorphism $\hat{k}^{\circ}\left\{T_{1}, \ldots, T_{m}\right\} \rightarrow A$. By Corollary 6.3 , we may assume that $\varepsilon$ is defined by the triple $\left(\mathfrak{X}_{\eta},\left\{f_{2}\right\},\left\{t_{\imath}\right\}\right)$ for some $t_{1}, \ldots, t_{m}>0$. We claim that any $n \geq 1$ such that $|a|^{n} \leq t_{2}$ for all $1 \leq i \leq m$ possesses the necessary property. Indeed, let $\varphi, \psi: \mathfrak{Y} \rightarrow \mathfrak{X}$ be a pair of morphisms that coincide modulo $a^{n}$. We may assume that $\mathfrak{Y}=\operatorname{Spf}(B)$, where $B$ is topologically finitely presented over $\widehat{k}^{\circ}$. Then $\varphi^{*} f_{2}-\psi^{*} f_{i}=a^{n} g_{2}$ for some $g_{2} \in B$. Since the image of $B$ in $\mathcal{B}:=B \otimes_{\hat{k}_{i}^{\circ}} \widehat{k}$ is contained in $\mathcal{B}^{\circ}$, it follows that $\rho\left(\varphi_{\eta}^{*} f_{\imath}-\psi_{\eta}^{*} f_{\imath}\right) \leq|a|^{n} \leq t_{\imath}, 1 \leq i \leq m$.

If $\mathfrak{X}$ is arbitrary, we take a finite covering $\left\{\mathfrak{X}_{\imath}\right\}_{\imath \in I}$ of $\mathfrak{X}$ by open affine formal subschemes of the above form. Then $\left\{\mathfrak{X}_{i, \eta}\right\}_{\imath} \in I$ is a finite affinoid covering $\mathfrak{X}_{\eta}$. By Proposition 6.1, we may assume that $\varepsilon$ is the infimum of $\varepsilon_{\imath}$ with $s\left(\varepsilon_{2}\right)=\left\{\mathfrak{X}_{2, \eta}\right\}$. The previous case applied to $\mathfrak{X}_{\imath}$ and $\varepsilon_{\imath}$ gives integers $n_{\imath} \geq 1, i \in I$. It is easy to see that $n:=\max _{\imath \in I} n_{\imath}$ satisfies the necessary property.

We are now ready to prove the theorem. First of all, we can replace $k$ by its maximal unramified extension and assume that the residue field $\widetilde{k}$ is separably closed. Let $0 \leq q \leq s_{l}(k)+2 \operatorname{dim}\left(\mathcal{X}_{\eta}\right)$. Since the sheaf $i^{*}\left(R^{q} j_{*}\left(\left.\mathcal{F}\right|_{\mathcal{X}_{\eta}}\right)\right)$ is constructible and is associated with the presheaf $\mathfrak{U}_{s} \mapsto H^{q}\left(\mathfrak{U}_{\eta}, \widehat{\mathcal{F}}\right)$, we can find a finite étale covering $\left\{\mathfrak{U}_{\nu, s} \stackrel{f_{\nu}}{\rightarrow} \mathcal{X}_{s}\right\}$ by separated schemes of finite type over $\tilde{k}$ such that if $\mathcal{G}_{\nu}$ denotes the constant sheaf on $\mathfrak{U}_{\nu, s}$ associated with the finite group $H^{q}\left(\mathfrak{U}_{\nu, \eta}, \widehat{\mathcal{F}}\right)$, then the canonical homomorphism $\oplus_{\nu} f_{\nu !}\left(\mathcal{G}_{\nu}\right) \rightarrow i^{*}\left(R^{q} j_{*}\left(\left.\mathcal{F}\right|_{\mathcal{X}_{\eta}}\right)\right)$ is surjective. By Theorem 7.1 and Lemma 8.4, we can find $n \geq 1$ such that, for each $\nu$ and any pair of morphisms of formal schemes $\varphi, \psi: \mathfrak{Y} \rightarrow \mathfrak{U}_{\nu}$ that coincide modulo $a^{n}$, the homomorphisms $\left.H^{q}\left(\mathfrak{U}_{\nu, \eta}, \widehat{\mathcal{F}}\right) \rightarrow H^{q}(\mathfrak{Y})_{\eta}, \widehat{\mathcal{F}}\right)$ induced by $\varphi$ and $\psi$ coincide. We claim that this $n$ satisfies the required property (for the chosen $q$ ).

Indeed, let $\varphi, \psi: \hat{\mathcal{Y}} \rightarrow \widehat{\mathcal{X}}$ be a pair of $\widehat{\mathcal{T}}$-morphisms that coincide modulo $a^{n}$. We set $\mathfrak{V}_{\nu}=\hat{\mathcal{Y}} \times \widehat{\mathcal{X}}_{, \varphi} \mathfrak{U}_{\nu}$ and denote by $\varphi_{\nu}$ and $g_{\nu}$ the induced morphisms $\mathfrak{V}_{v} \rightarrow \mathfrak{U}_{v}$, and $\mathfrak{V}_{t} \rightarrow \widehat{\mathcal{Y}}$, respectively. Since $\varphi$ and $\psi$ coincide modulo $a^{n}$, there is a canonical isomorphism $\mathfrak{V}_{\nu, n} \stackrel{\sim}{\rightarrow}\left(\widehat{\mathcal{Y}} \times \widehat{\chi}_{, \psi} \mathfrak{H}_{\nu}\right)_{n}$. By Lemma 2.1 , it induces an isomorphism $\mathfrak{V}_{\nu} \stackrel{\sim}{\rightarrow} \hat{\mathcal{Y}} \times \hat{\mathcal{X}}_{, \psi}, \mathfrak{U}_{\nu}$. Let $\psi_{\nu}$, denote the composition of the latter isomorphism with the canonical morphism $\widehat{\mathcal{Y}} \times \widehat{\boldsymbol{x}}_{, \psi} \mathfrak{U}_{\nu} \rightarrow \mathfrak{U}_{\nu}$. Thus, we get two morphisms $\varphi_{\nu}, \psi_{\nu}: \mathfrak{V}_{\nu} \rightarrow \mathfrak{U}_{\nu}$ that coincide modulo $a^{n}$ and extend the morphisms $\varphi, \psi: \widehat{\mathcal{Y}} \rightarrow \hat{\mathcal{X}}$, respectively. By our choice of $n$, the homomorphisms $H^{q}\left(\mathfrak{U}_{\nu, \eta}, \widehat{\mathcal{F}}\right) \rightarrow H^{q}\left(\mathfrak{V}_{\nu, \eta}, \widehat{\mathcal{F}}\right)$ induced by $\varphi_{\nu}$ and $\psi_{\nu}$ coincide. If $\mathcal{H}_{\nu}$ denotes the constant sheaf on $\mathfrak{V}_{\nu, s}$ associated with the group $H^{q}\left(\mathfrak{V}_{\nu, \eta}, \widehat{\mathcal{F}}\right)$, then the latter means that the homomorphisms of sheaves $\varphi_{\nu, s}^{*}\left(\mathcal{G}_{\nu}\right)=\psi_{\nu, s}^{*}\left(\mathcal{G}_{\nu}\right) \rightarrow \mathcal{H}_{\nu}$ induced by $\varphi_{\nu}$ and $\psi_{\nu}$ coincide. Furthermore, for each $\nu$ there is a commutative diagram 


$$
\begin{array}{ccc}
\varphi_{s}^{*}\left(f_{\nu !}\left(\mathcal{G}_{\nu}\right)\right)=\psi_{s}^{*}\left(f_{\nu !}\left(\mathcal{G}_{\nu}\right)\right) & \longrightarrow & \varphi_{s}^{*}\left(i^{*}\left(R^{q} j_{*}\left(\left.\mathcal{F}\right|_{\mathcal{X}_{\eta}}\right)\right)\right)=\psi_{s}^{*}\left(i^{*}\left(R^{q} j_{*}\left(\left.\mathcal{F}\right|_{\mathcal{X}_{\eta}}\right)\right)\right) \\
\theta_{\nu, \varphi}||_{g_{\nu, \psi}} & & \theta^{q}(\varphi, \mathcal{F}) \mid \\
g_{\nu !}\left(\mathcal{H}_{\nu}\right) & \longrightarrow & \theta^{q}(\psi, \mathcal{F})
\end{array}
$$

where $\theta_{\nu, \varphi}: \varphi_{s}^{*}\left(f_{\nu !}\left(\mathcal{G}_{\nu}\right)\right)=g_{\nu !}\left(\varphi_{\nu, s}^{*} \mathcal{G}_{\nu}\right) \rightarrow g_{\nu !}\left(\mathcal{H}_{\nu}\right)$ and $\theta_{\nu, \psi}: \psi_{s}^{*}\left(f_{\nu !}\left(\mathcal{G}_{\nu}\right)\right)=$ $g_{\nu !}\left(\psi_{\nu, s}^{*} \mathcal{G}_{\nu}\right) \rightarrow g_{\nu !}\left(\mathcal{H}_{\nu}\right)$. Since $\dot{\theta}_{\nu, \varphi}=\theta_{\nu, \psi}$ for all $\nu$, it follows that $\theta^{q}(\varphi, \mathcal{F})=$ $\theta^{q}(\psi, \mathcal{F})$. The theorem is proved.

Corollary 8.5. Let $\mathcal{F}$ be a constructible sheaf on $\mathcal{T}_{\eta}$ with torsion orders prime to $\operatorname{char}(\widetilde{k})$. Given $\hat{\mathcal{X}}$, where $\mathcal{X}$ is a scheme over $\mathcal{T}$, there exists $n \geq 1$ such that, for any scheme $\mathcal{Y}$ over $\mathcal{T}$ and any pair of $\widehat{\mathcal{T}}$-morphisms $\varphi, \psi: \widehat{\mathcal{Y}} \rightarrow \widehat{\mathcal{X}}$ that coincide modulo $a^{n}$, one has $\theta_{\eta}^{q}(\varphi, \mathcal{F})=\theta_{\eta}^{q}(\psi, \mathcal{F})$ for all $q \geq 0$.

For a scheme $\mathcal{X}$ over $\mathcal{T}$, we denote by $\mathcal{G}(\widehat{\mathcal{X}} / \widehat{\mathcal{T}})$ the group of $\widehat{\mathcal{T}}$-automorphisms of $\hat{\mathcal{X}}$ and by $\mathcal{G}_{n}(\hat{\mathcal{X}} / \widehat{\mathcal{T}})$ its subgroup consisting of the automorphisms trivial modulo $a^{n}$. Furthermore, recall that a $\mathbf{Z}_{l}$-sheaf on a Noetherian scheme is a projective system of étale constructible $\mathbf{Z} / l^{m+1} \mathbf{Z}$-modules $\mathcal{F}_{m}, m \geq 0$, such that, for each $m \geq 1$, the canonical homomorphism $\mathcal{F}_{m} \rightarrow \mathcal{F}_{m-1}$ induces an isomorphism $\mathcal{F}_{m} \otimes_{\mathbf{Z} / l^{m+1} \mathbf{Z}}$ $\mathbf{Z} / l^{m} \mathbf{Z} \stackrel{\sim}{\rightarrow} \mathcal{F}_{m-1}$ (see [SGA4 $\frac{1}{2}$ ], Rapport, §2).

Theorem 8.6. Let $\mathcal{F}$ be a $\mathbf{Z}_{l}$-sheaf on $\mathcal{T}_{\eta}, l \neq \operatorname{char}(\tilde{k})$, and suppose that $s_{l}(k)<\infty$. Given $\widehat{\mathcal{X}}$, where $\mathcal{X}$ is a scheme over $\mathcal{T}$, there exists $n \geq 1$ such that the group $\mathcal{G}_{n}(\widehat{\mathcal{X}} / \widehat{\mathcal{T}})$ acts trivially on all of the sheaves $i^{*}\left(R^{q} j_{*} \mathcal{F}_{m}\right), q \geq 0, m \geq 0$.

Lemma 8.7. Let $R$ be a commutative ring, $A$ a commutative $R$-algebra complete in the a-adic topology for some ideal $\mathbf{a} \subset A, \mathcal{G}(A / R)$ the group of the automorphisms $\sigma$ of $A$ over $R$ with $\sigma(\mathbf{a})=\mathbf{a}, \mathcal{G}_{n}(A / R)$ the subgroup of automorphisms trivial modulo $\mathbf{a}^{n}$. If a prime number $l$ is invertible in $A$, then for any $n \geq 2$ (resp. $n \geq 1$ if $\mathbf{a}$ is generated by elements of $R)$ the group $\mathcal{G}_{n}(A / R)$ is uniquely l-divisible.

Proof. Let $E$ be the ring of the endomorphisms $\varphi$ of the $R$-module $A$ with $\varphi\left(\mathbf{a}^{m}\right) \subset$ $\mathbf{a}^{m}$ for all $m \geq 0$. For $\varphi \neq 0$, let $w(\varphi)$ denote the maximal integer $\nu$ such that $\varphi\left(\mathbf{a}^{m}\right) \subset$ $\mathbf{a}^{m+\nu}$ for all $m \geq 0$. We also set $w(0)=\infty$. One has $w(\varphi+\psi) \geq \inf \{w(\varphi), w(\psi)\}$ and $w(\varphi \psi) \geq w(\varphi)+w(\psi)$, i.e., $w$ is a filtration on $E$. In particular, $E_{m}:=\{\varphi \in$ $E \mid w(\varphi) \geq m\}$ are two sided ideals in $E$. Since $A$ is a-adic, the ring $E$ is separated and complete in the topology defined by the ideals $E_{m}$. It follows that $U_{m}:=1+E_{m}$, $m \geq 1$, are subgroups of $E^{*}$. One has $\mathcal{G}_{n}(A / R) \subset U_{n-1}\left(\right.$ resp. $\left.\mathcal{G}_{n}(A / R) \subset U_{n}\right)$.

We claim that, for any $n \geq 1$, the group $U_{n}$ is uniquely $l$-divisible. Indeed, if $\varphi \in E_{n}$, then the series $\sqrt[l]{1+\varphi}=\sum_{q=0}^{\infty}\left(\begin{array}{l}\frac{1}{l} \\ q\end{array}\right) \varphi^{q}$ is convergent in $E$, and therefore $U_{n}$ is $l$-divisible. Suppose that $(1+\varphi)^{l}=(1+\psi)^{l}$ for some $\varphi \neq \psi$ in $E_{1}$. Then $w(\varphi)=w(\psi)$ and $l(\varphi-\psi)=\frac{l(l-1)}{2}\left(\psi^{2}-\varphi^{2}\right)+\ldots+\left(\psi^{l}-\varphi^{l}\right)$. For each $i \geq 2$, one has $w\left(\psi^{2}-\varphi^{2}\right) \geq(i-1) w(\varphi)+w(\varphi-\psi)>w(\varphi-\psi)$. The latter contradicts to the above equality.

It remains to verify that if $\sigma \in U_{1} \cap \mathcal{G}(A / R)$, then $\tau:=\sqrt[b]{\sigma} \in \mathcal{G}(A / R)$, i.e., $\tau(a b)=\tau(a) \tau(b)$ for all $a, b \in A$. If $\sigma=1+\varphi$, where $\varphi \in E_{1}$, then the latter is equivalent to the equality 


$$
\sum_{q=0}^{\infty}\left(\begin{array}{c}
\frac{1}{l} \\
q
\end{array}\right) \varphi^{q}(a b)=\sum_{i, j=0}^{\infty}\left(\begin{array}{l}
\frac{1}{l} \\
i
\end{array}\right)\left(\begin{array}{l}
\frac{1}{l} \\
j
\end{array}\right) \varphi^{2}(a) \varphi^{\jmath}(b)
$$

Since $\sigma^{m}(a b)=\sigma^{m}(a) \sigma^{m}(b), m \geq 0$, then there are equalities

$$
\sum_{q=0}^{m}\left(\begin{array}{c}
m \\
q
\end{array}\right) \varphi^{q}(a b)=\sum_{\imath, \jmath=0}^{m}\left(\begin{array}{c}
m \\
i
\end{array}\right)\left(\begin{array}{c}
m \\
j
\end{array}\right) \varphi^{\imath}(a) \varphi^{\jmath}(b) \text {. }
$$

We introduce inductively by $m \geq 0$ polynomials $f_{m} \in \mathbf{Z}\left[X_{0}, \ldots, X_{m}, Y_{0}, \ldots, Y_{m}\right]$ by the equalities

$$
\sum_{q=0}^{m}\left(\begin{array}{c}
m \\
q
\end{array}\right) f_{q}=\sum_{i, \jmath=0}^{m}\left(\begin{array}{c}
m \\
i
\end{array}\right)\left(\begin{array}{c}
m \\
j
\end{array}\right) X_{\imath} Y_{j}
$$

Then $\varphi^{m}(a b)=f_{m}\left(a, \ldots, \varphi^{m}(a), b, \ldots, \varphi^{m}(b)\right)$. Let $B$ be the ring of polynomials $\mathbf{Z}\left[\frac{1}{l}\right]\left[X_{0}, X_{1}, \ldots, Y_{0}, Y_{1} \ldots\right]$ graduated by $\operatorname{deg}\left(X_{2}\right)=\operatorname{deg}\left(Y_{\imath}\right)=i$. For a polynomial $f \in B$, let $e(f)$ denote the minimal degree of a monomial that enters in $f$. For example, $e\left(f_{m}\right)=m$ (since $f_{m}=\sum_{i=0}^{m} \sum_{\jmath=0}^{m-\imath}\left(\begin{array}{c}m \\ i\end{array}\right)\left(\begin{array}{c}m-i \\ j\end{array}\right) X_{t+j} Y_{m-\jmath}$ ). Let $\widehat{B}$ be the completion $\underbrace{\lim }_{m} B / \mathbf{b}_{m}$, where $\mathbf{b}_{m}$ is the ideal $\{f \in B \mid e(f) \geq m\}$. The equality (?) follows from the following equality in $\widehat{B}$

$$
\sum_{q=0}^{\infty}\left(\begin{array}{c}
\frac{1}{l} \\
q
\end{array}\right) f_{q}=\sum_{n, j=0}^{\infty}\left(\begin{array}{l}
\frac{1}{l} \\
i
\end{array}\right)\left(\begin{array}{l}
\frac{1}{l} \\
j
\end{array}\right) X_{\imath} Y_{j}
$$

Let $p$ be a prime number different from $l$, and let $m$ be the integer with $m l \equiv 1(\bmod p)$ and $1 \leq m \leq p-1$. Then $\left(\begin{array}{c}\frac{1}{l} \\ i\end{array}\right) \equiv\left(\begin{array}{c}m \\ i\end{array}\right)(\bmod p)$ for all $0 \leq i \leq p-1$, and therefore the equality $\left(?^{\prime}\right)$ is congruent to the equality $\left(*^{\prime}\right)$ modulo the ideal generated by $p$ and $\mathbf{b}_{m+1}$. Note that $m \geq\left[\frac{p+1}{l}\right]$, and therefore $m \rightarrow \infty$ when $p \rightarrow \infty$. It follows that $\left(?^{\prime}\right)$ is true.

Proof of Theorem 8.6. There is an exact sequence of $\mathrm{Z}_{l}$-sheaves $0 \rightarrow \mathcal{F}^{\prime} \rightarrow \mathcal{F} \rightarrow$ $\mathcal{F}^{\prime \prime} \rightarrow 0$ such that $l^{\nu} \mathcal{F}^{\prime}=0$ for some $\nu \geq 0$ and $\mathcal{F}^{\prime \prime}$ is without torsion (see [SGA $4 \frac{1}{2}$ ], Rapport, 2.8). It induces, for each $m \geq 0$, an exact sequence $0 \rightarrow \mathcal{F}_{m}^{\prime} \rightarrow \mathcal{F}_{m} \rightarrow$ $\mathcal{F}_{m}^{\prime \prime} \rightarrow 0$. We also remark that $\mathcal{F}_{m}^{\prime} \stackrel{\sim}{\rightarrow} \mathcal{F}_{\nu}^{\prime}$ for $m \geq \nu$ and that, for each $m \geq 1$, there exists an exact sequence $0 \rightarrow \mathcal{F}_{m-1}^{\prime \prime} \rightarrow \mathcal{F}_{m}^{\prime \prime} \rightarrow \mathcal{F}_{1}^{\prime \prime} \rightarrow 0$. By Theorem 8.1 , we can find $n \geq 1$ such that the group $\mathcal{G}_{n}(\widehat{\mathcal{X}} / \widehat{\mathcal{T}})$ acts trivially on all of the sheaves $i^{*}\left(R^{q} j_{*} \mathcal{F}_{m}^{\prime}\right)$, $0 \leq m \leq \nu$, and $i^{*}\left(R^{q} j_{*} \mathcal{F}_{1}^{\prime \prime}\right)$. By Lemma 8.7 , this group is uniquely $l$-divisible. It follows that it acts trivially on all of the sheaves $i^{*}\left(R^{q} j_{*} \mathcal{F}_{m}\right), q \geq 0, m \geq 0$.

Corollary 8.8. Let $\mathcal{F}$ be a $\mathbf{Z}_{l}$-sheaf on $\mathcal{T}_{\eta}, l \neq \operatorname{char}(\tilde{k})$. Given $\widehat{\mathcal{X}}$, where $\mathcal{X}$ is a scheme over $\mathcal{T}$, there exists $n \geq 1$ such that the group $\mathcal{G}_{n}(\widehat{\mathcal{X}} / \widehat{\mathcal{T}})$ acts trivially on all of the sheaves $R^{q} \Psi_{\eta}\left(\mathcal{F}_{m}\right), q \geq 0, m \geq 0$. 


\section{References}

[Berl] Berkovich, V. G.: Spectral theory and analytic geometıy over non-Archimedean fields, Mathematical Surveys and Monographs, vol. 33, American Mathematical Society, 1990

[Ber2] Berkovich, V. G.: Étale cohomology for non-Archimedean analytic spaces, Publ. Math. IHES 78, 5-161 (1994)

[Ber3] Berkovich, V. G.: Vanishing cycles for non-Archimedean analytic spaces, (submitted to Journal of the AMS)

[BGR] Bosch, S; Güntzer, U.; Remmert, R.: Non-Archimedean analysis. A systematic approach to rigid analytic geometry, Grundlehren der Mathematischen Wissenschaften, Bd. 261, Springer, Berlin-Heidelberg-New York, 1984

[EGA1] Grothendieck, A.; Dieudonné, J.: Eléments de Géométrie Algébrique. I. Le langage des schémas, Springer, Berlin-Heidelberg-New York, 1971

[God] Godement, R.: Topologie Algébrique et Théorie des Faisceux, Hermann, Paris, 1958

[Kel] Kelley, J. L.: General Topology, D. Van Nostrand Company, Toronto-London-New York, 1957

[Ray] Raynaud, M.: Anneaux Locaux Henséliens, Lecture Notes in Math. 169, Springer, BerlinHeidelberg-New York, 1970

[SGA1] Grothendieck, A.: Séminaire de Géométrie Algébrique. I. Revêtements étales et Groupe Fondemental, Lecture Notes in Math. 224, Springer, Berlin-Heidelberg-New York, 1971

[SGA4] Artin, M., Grothendieck, A., Verdier, J.-L.: Théorie des Topos et Cohomologie Étale des Schémas, Lecture Notes in Math. 269, 270, 305, Springer, Berlin-Heidelberg-New York, 1972 1973

[SGA4 $\frac{1}{2}$ ] Deligne, P. et al.: Cohomologie Étale, Lecture Notes in Math. 569, Springer, Berlin-HeidelbergNew York, 1977

[SGA7] Grothendieck, A., Deligne, P., Katz, N.: Groupes de Monodromie en Géométrie Algébrique, Lecture Notes in Math. 288, 340, Springer, Berlin-Heidelberg-New York, 1972-1973

This article was processed by the author

using the Springer-Verlag TEX Invent macro package 1991. 